\title{
On the classification of simple stably projectionless $\mathrm{C}^{*}$-algebras
}

\author{
by
}

\section{Shaloub Razak}

A thesis submitted in conformity with the requirements for the degree of Phd.

Graduate Department of Mathematics

University of Toronto

(C) Copyright by Shaloub Razak, August 2000 
National Library

of Canada

Acquisitions and Bibliographic Services

395 Wellington Street Otawa ON KTA ONA Canada
Bibliothèque nationale du Canada

Acquisitions et senvices bibliographiques

395, ne Wellington

Otenva ON KTA ONA

Canada
The author has granted a nonexclusive licence allowing the National Library of Canada to reproduce, loan, distribute or sell copies of this thesis in microform, paper or electronic formats.

The author retains ownership of the copyright in this thesis. Neither the thesis nor substantial extracts from it may be printed or otherwise reproduced without the author's permission.
L'auteur a accordé une licence non exclusive permettant à la Bibliothèque nationale du Canada de reproduire, prêter, distribuer ou vendre des copies de cette thèse sous la forme de microfiche/film, de reproduction sur papier ou sur format électronique.

L'auteur conserve la propriété du droit d'auteur qui protège cette thèse. $\mathrm{Ni}$ la thèse ni des extraits substantiels de celle-ci ne doivent être imprimés ou autrement reproduits sans son autorisation.

\section{Canadầ}


On the classification of simple stably projectionless $\mathrm{C}^{*}$-algebras, Phd. thesis, August 2000, Shaloub Razak, Department of Mathematics, University of Toronto

\begin{abstract}
It is shown that simple stably projectionless $\mathrm{C}^{*}$-algebras which a re inductive limits of certain specified building blocks with trivial K-theory are classified by their cone of positive traces with distinguished subset. This is the first example of an isomorphism theorem verifying the conjecture of Elliott for a subclass of the stably projectionless algebras.
\end{abstract}




\section{Acknowledgments}

I would like to thank David Kerr for introducing me to the beautiful subject of $\mathrm{C}^{*}$-algebras and Marek Stastna for many helpful discussions on matters mathematical, physical and otherwise. I would also like to thank my family for their support during my doctoral studies and long before. Finally, I acknowledge with gratitude the guidance and encouragement of my thesis advisor, George A. Elliott. 


\section{Contents}

1 Introduction 1

1.1 The building blocks ................. 3

2 The invariant 5

2.1 Intertwining the invariant ............. 9

2.2 Isomorphism .................... 24

3 Existence 20

3.1 The desingularized space ................ 30

3.2 The rescaled map ................. . . . 34

3.3 Lifting to $M_{(a+1)^{2} p}\left(C\left(X^{\sharp}\right)\right) \ldots \ldots . \ldots . \ldots . \ldots$

4 Uniqueness $\quad 54$

4.1 Constructing the standard maps . . . . . . . . . . . 59

4.2 Comparing maps in standard form .......... 73

4.3 Endpoint considerations .................... 104

4.4 Jiang and Su's argument ... . . . . . . . . . . 124 


\section{Chapter 1}

\section{Introduction}

During the last decade the classification program for amenable $C^{*}$-algebras has been a very active field of mathematical research both internationally and within Canada. This thesis is a contribution to the ongoing program to classify and better understand these algebras.

Invariants are important in the process of classification because they distinguish in an essential manner between objects. The K-theoretical invariant proposed by Elliott in [Ell5] to classify stable simple amenable $\mathrm{C}^{*}$-algebras has been very successful in dividing the problem into three broad classes, as follows.

Case (1): $K_{0}^{+}=0 ; T^{+} \neq 0$.

Case (2): $K_{0}^{+} \cap-K_{0}^{+}=0, K_{0}^{+}-K_{0}^{+}=K_{0} \neq 0 ; T^{+} \neq 0$.

Case (3): $\mathrm{K}_{0}^{+}=\mathrm{K}_{0} ; \mathrm{T}^{+}=0$.

The construction of the $\mathrm{K}_{0}$ group of a $\mathrm{C}^{*}$-algebra can be described as 
follows. The equivalence class of projections in a stable $C^{*}$-algebra forms a semigroup under addition. Two projections, $p$ and $q$ are considered equivalent if there is an algebra element $v$ such that $p=v^{*} v$ and $q=v v^{*}$. The $\mathrm{K}_{0}$ group is the enveloping group of the semigroup of projections (if the algebra is not the stabilization of a unital algebra then the given construction should be suitably modified).

Furthermore, the $K_{0}$ group has a natural pre-order structure; $K_{0}^{+}$is the positive cone consisting of projections in the algebra. The space $\mathrm{T}^{+}$of densely defined, lower semicontinuous, positive traces has a natural structure as a topological convex cone (the topology considered is the $\mathrm{w}^{*}$-topology induced by the Pedersen ideal). In addition to $\mathrm{K}_{0}$ and $\mathrm{T}^{+}$, the proposed invariant also includes the abelian group $K_{1}$ (the group of homotopy classes of unitaries in the algebra), and the natural pairing of the cone of traces with $K_{0}$.

The breakthrough of Kirchberg [Kir] and Phillips [Phi] (based on earlier work of Kirchberg's) in the classification of "purely infinite" algebras has all but exhausted Case (3) of the program (a technical restriction still remains). Numerous classification results have been obtained in Case (2). The work of Elliott, Gong, and Li [EGL] in classifying algebras composed of simpler building blocks - matrix algebras over arbitrary compact metrizable spaces of bounded finite dimension - is the most general result known so far in Case (2). The first classification result obtained in Case (1), the class of stably projectionless $\mathrm{C}^{*}$-algebras, is the candidate's doctoral thesis.

The first example of a simple stably projectionless $\mathrm{C}^{*}$-algebra was given by Blackadar in [Bla]. In recent work of Dean [Dea] it was shown that a class of simple stably projectionless $\mathrm{C}^{*}$-algebras constructed by Kishimoto and 
Kumjian in $[\mathrm{KK}]$ can be decomposed into simpler building blocks. The result proven in this thesis is that simple stably projectionless algebras composed of certain building blocks are classified by their cone of positive traces with the distinguished subset of traces of norm at most one.

A more explicit description is as follows.

Theorem 1.0.1 Let $A$ and $B$ be simple inductive limits of building block algebras with injective connecting maps. If $\left(\mathrm{T}^{+} A, \Sigma_{A}\right)$ is isomorphic to $\left(\mathrm{T}^{+} B, \Sigma_{B}\right)$ then $A$ is isomorphic to $B$.

The building block algebras that are considered are defined in Section 1.1. The compact set $\Sigma$ is the subset of $\mathrm{T}^{+}$of traces of norm at most one.

\section{$1.1 \quad$ The building blocks}

The building blocks that are considered are certain subhomogeneous algebras (of the full matrix algebra over the interval) obtained by a generalized mapping torus construction as in [EV] and [Ell6]. Specifically, given a pair of simple matrix algebras $C$ and $D$, a positive integer $a$, a pair of homomorphisms $L$ and $R$ from $C$ to $D$ with multiplicities $a$ and $a+1$ respectively (we will assume that $R$ is a unital map), the associated building block algebra has the form:

$$
\begin{aligned}
A & =A(C, D, a) \\
& :=\{(c, d) ; c \in C, d \in \mathrm{C}([0,1] ; D), d(0)=L(c), d(1)=R(c)\} .
\end{aligned}
$$

The building block $A$ is an extension of the suspension $S D$ of $D$ by $C$

$$
0 \rightarrow \mathcal{S} D \rightarrow A \rightarrow C \rightarrow 0 .
$$


The six term exact sequence in $\mathrm{K}$-theory applied to the short exact sequence above yields $\mathrm{K}_{0} A=\mathrm{K}_{1} A=0$.

We can describe all irreducible representations of a building block algebra in terms of "evaluation maps". Let $e_{s}: A \rightarrow D$ denote the representation of the building block algebra $A$ obtained by evaluating at $s, 0 \leq s \leq 1$. For $s \in(0,1)$ the evaluation map is an irreducible representation. Let $e_{\infty}: A \rightarrow$ $C$ denote the representation of $A$ obtained by evaluating at the irreducible fibre at infinity. Every irreducible representation of $A$ is unitarily equivalent to $e_{s}$ for $s \in(0,1)$ or $e_{\infty}$. 


\section{Chapter 2}

\section{The invariant}

The invariant consists of the cone of positive traces $T^{+}$endowed with the weak-* topology, together with the distinguished subset $\Sigma$ of traces of norm less than or equal to one. The dual of the invariant is the space $\left(\mathrm{Aff} T^{+},\|\cdot\|\right)$ of continuous affine functions on $T^{+}$, with the semi-norm $\|\cdot\|$ given by the supremum of the restriction of an affine function to the compact set $\Sigma$. (If all the traces of the algebra are bounded then $\|\cdot\|$ is a proper norm.) Isometric isomorphisms (with respect to the semi-norm) of the dual of the invariant are canonically equivalent to isomorphisms of the invariant. (In [Ste] a similar invariant is used to classify certain simple inductive limit algebras.)

Here is an equivalent dual formulation of theorem 1.0.1, the isomorphism theorem:

Theorem 2.0.1 Let $A$ and $B$ be simple inductive limits of building blocks with injective connecting maps. If $\left(\mathrm{Aff} T^{+} A,\|\cdot\|_{A}\right)$ is isomorphic to (Aff $T^{+} B,\|\cdot\|_{B}$ ) then $A$ is isomorphic to $B$.

In the remaining part of this section two different norms will be introduced on 
the space of affine functions of a building block algebra. In each case it will be shown that the space of affine functions can be identified isometrically with a certain subspace of the positive real valued functions on the unit interval (which will be denoted as $C[0,1]$ ) endowed with the sup-norm.

All traces on a building block algebra $A(C, D, a)$ are bounded and so $\Sigma_{A}$ induces a (proper) norm on the space of affine functions as follows:

$$
\|f\|_{A}=\sup \left\{f(\tau): \tau \in \Sigma_{A}\right\}, f \in \operatorname{Aff} T^{+} A
$$

where $\Sigma_{A}$ is the compact set defined as

$$
\Sigma_{A}=\left\{\tau \in T^{+} A:\|\tau\| \leq 1\right\}
$$

Let $A=\lim _{\rightarrow}\left(A_{i}, \phi_{i j}\right)$. Then it is easy to check that

$$
\left\|\phi_{i \infty 0 *}(f)\right\|_{A}=\lim _{j \rightarrow \infty}\left\|\phi_{i j *}(f)\right\|_{A_{j}},
$$

where $f \in \operatorname{Aff} T^{+} A_{j}$ (the maps $\phi_{i j *}$ between the affine function spaces are induced by the *-homomorphisms $\phi_{i j}$ ). Let $C[0,1]_{a}$ denote the subspace of $C[0,1]$ defined as

$$
C[0,1]_{a}=\left\{f \in C[0,1]: f(0)=\frac{a}{a+1} f(1)\right\} .
$$

Proposition 2.0.1 Let $A(C, D, a)$ be a building block algebra. Then there exists an isometric isomorphism $\iota:\left(\operatorname{Aff} T^{+} A,\|\cdot\|_{A}\right) \rightarrow\left(C[0,1]_{a},\|\cdot\|_{\infty}\right)$. Furthermore, $\inf \left\{f(\tau): \tau \in T^{+} A,\|\tau\|=1\right\}=\inf \iota(f)$ for all $f \in \mathrm{Aff}^{+} A$.

Proof. Every trace on a building block $A$ has the form $\operatorname{tr} \otimes \mu$, where $\operatorname{tr}$ is the usual normalized matrix trace, and $\mu$ is a finite measure on $(0,1]$. The extreme traces of norm equal to one are parameterized by $t \in(0,1]$, and are 
given as $\operatorname{tr} \otimes \delta_{t}$, where $\delta_{t}$ is the normalized point mass at $t$. In the weak-* topology, as $t \rightarrow 0, \operatorname{tr} \otimes \delta_{t} \rightarrow \frac{a}{a+1} \operatorname{tr} \otimes \delta_{1}$. Therefore the map

$$
\begin{aligned}
\iota:\left(\operatorname{Aff}^{+} A,\|\cdot\|_{A}\right) & \rightarrow\left(C[0,1]_{a},\|\cdot\|_{\infty}\right) \\
f & \mapsto t r \otimes \delta_{t}(f)=\iota(f)(t)
\end{aligned}
$$

establishes an isometric isomorphism between the two spaces. Since the infimum of an affine function occurs on the set of extreme traces, the second part of the proposition follows directly from the definition of the map $\iota$.

A second norm can be introduced on the building block algebra $A(C, D, a)$ as follows: given a function $n \in \operatorname{Aff} T^{+} A$ such that inf $\iota(n)>0$ (with the map $\iota$ as defined in Proposition 2.0.1), define the closed convex set

$$
\Sigma_{n}=\left\{\tau \in T^{+} A: n(\tau)=1\right\}
$$

Let

$$
\|f\|_{n}=\sup \left\{f(\tau): \tau \in \Sigma_{n}\right\}
$$

Clearly, the set $\Sigma_{n}$ is closed in the weak-* topology. The fact that it is compact follows immediately from the following proposition:

Proposition 2.0.2 Let $A(C, D, a)$ be a building block algebra and $n \in \mathrm{Aff}^{+} A$ be an element of its space of affine functions such that $\|n\|_{A} \leq 1$. If $\inf \iota(n)=$ $\alpha>0$ then $\Sigma_{n} \subset \frac{1}{\alpha} \Sigma_{A}$.

Proof. Let us show that $\|\tau\| \leq 1 / \alpha$ for all $\tau \in \Sigma_{n}$. Let $f$ be an affine function such that $\|f\|_{A} \leq 1$. By Proposition 2.0.1, for all traces $\tau \in T^{+} A$ of norm equal to one, $n(\tau) \geq \alpha$, and hence

$$
\alpha f(\tau) \leq n(\tau) \text { for all } \tau \in T^{+} A
$$


Then for any $\tau \in \Sigma_{n}, \tau(f) \leq \tau(n) / \alpha \leq 1 / \alpha$, and therefore $\|\tau\| \leq 1 / \alpha$.

For the remainder of this section $\left(C(\mathbb{T}),\|\cdot\|_{\infty}\right)$, the space of positive real valued functions over the circle, will be identified with the subspace $\left(\{f \in C[0,1]: f(0)=f(1)\},\|\cdot\|_{\infty}\right)$. The proof of the following proposition is similar to that of Proposition 2.0.1.

Proposition 2.0.3 Let $A(C, D, a)$ be a building block algebra and $n \in \mathrm{AffT}^{+} A$ such that $\inf \iota(n)>0$ (where $\iota$ denotes the map defined in Proposition 2.0.1). Then (Aff $\left(T^{+} A,\|\cdot\|_{n}\right)$ is isometrically isomorphic to $\left(C(\mathbb{T}),\|\cdot\|_{\infty}\right)$ by an isomorphism that carries $n$ to the unit element of $C(\mathbb{T})$. The isomorphism can be concretely realized as follows:

$$
\begin{aligned}
\iota_{n}^{\prime}:\left(\operatorname{Aff}\left(T^{+} A_{\imath}\|\cdot\|_{n}\right)\right) & \rightarrow\left(C(\mathbb{T}),\|\cdot\|_{\infty}\right) \\
f & \mapsto \iota(f) / \iota(n) .
\end{aligned}
$$

Furthermore, $\inf \left\{f(\tau): \tau \in \Sigma_{n}\right\}=\inf \iota_{n}^{\prime}(f)$.

Proof. First let's check that the image of $\iota_{n}^{\prime}$ lies in the subspace of $C[0,1]$ that has been identified with $C(\mathbb{T})$ by showing that the values of the function $\iota(f) / \iota(n) \in C[0,1]$ are equal at the endpoints:

$$
\frac{\iota(f)}{\iota(n)}(0)=\frac{\iota(f)(0)}{\iota(n)(0)}=\left(\frac{a}{a+1} \iota(f)(1)\right) /\left(\frac{a}{a+1} \iota(n)(1)\right)=\frac{\iota(f)}{\iota(n)}(1) .
$$

Since $\inf _{\iota}(n)>0$ it follows from Proposition 2.0.2 that $\Sigma_{n}=\left\{\tau \in T^{+} A\right.$ : $n(\tau)=1\}$ is a compact base for the cone $T^{+} A$. We claim that the extreme traces of $\Sigma_{n}$ are parameterized by $t \in(0,1]$, and are given as

$$
\frac{1}{\iota(n)(t)} \operatorname{tr} \otimes \delta_{t}
$$


where $\delta_{t}$ is the normalized point mass at $t$. To see that this is true it suffices to show that every such trace is actually in $\Sigma_{n}$ (clearly every extreme ray of $T^{+} A$ is a multiple of one of these traces);

$$
n\left(\frac{1}{\iota(n)(t)} \operatorname{tr} \otimes \delta_{t}\right)=\frac{1}{\iota(n)(t)} n\left(\operatorname{tr} \otimes \delta_{t}\right)=\frac{\iota(n)(t)}{\iota(n)(t)}=1
$$

Therefore the map

$$
\begin{aligned}
\iota_{n}^{\prime}:\left(\operatorname{Aff} T^{+} A,\|\cdot\|_{n}\right) & \rightarrow C(\mathbb{T}) \\
f & \mapsto f\left(\frac{1}{\iota(n)(t)} \operatorname{tr} \otimes \delta_{t}\right)=\frac{\iota(f)}{\iota(n)}(t)
\end{aligned}
$$

establishes an isometric isomorphism between the two spaces. The statement equating the infimum of $f$ on the compact convex set $\Sigma_{n}$ to the infimum of $\iota_{n}^{\prime}(f)$ follows immediately from the definition of the map $\iota_{n}^{\prime}$.

\subsection{Intertwining the invariant}

In section 2 two different norms were introduced on the space of affine functions of a building block algebra. As noted in section 2, the norms $\|\cdot\|_{A_{i}}$ defined by equation (2.1) extend continuously to a semi-norm on the inductive limit algebra by equation (2.2). In the first part of this section the norms $\|\cdot\|_{n_{i}}$ induced by certain elements $n_{i} \in \mathrm{Aff} T^{+} A_{i}$ and defined by equation (2.3) will be extended continuously to a norm on the inductive limit algebra.

In [Ell3] an intertwining of the invariant is obtained by factoring the identity map through a finite dimensional normed space, approximately on finitely many elements at each finite stage. In our setting the approximation 
will be made with respect to the two different norms at each finite stage. In the second part of this section these results are established.

Let $\left(A_{i}, \phi_{i j}\right)$ be an inductive limit system of building blocks $A_{i}\left(C_{i}, D_{i}, a_{i}\right)$ with inductive limit $A$. There are induced maps $\phi_{i j}^{*}: T^{+} A_{j} \rightarrow T^{+} A_{i}$ and $\phi_{i j *}:$ Aff $T^{+} A_{i} \rightarrow \operatorname{Aff} T^{+} A_{j}$ between the tracial cones and the affine function spaces, respectively, for $j>i$.

Let $n_{1} \in A f f T^{+} A_{1}$ be any function such that $\inf \iota\left(n_{1}\right)>0$. Given an inductive limit system $\left(A_{i}, \phi_{i j}\right)$, define $n_{i}=\phi_{1 i *}\left(n_{1}\right)$. The following proposition ensures that if $\inf \iota\left(n_{1}\right)>0$ then $\inf \iota\left(n_{j}\right)>0$ for all $j>i$ (this condition is necessary to define a coherent family of norms $\|\cdot\|_{n_{i}}$ on the inductive limit system).

Proposition 2.1.1 Let $f \in \mathrm{Aff}^{+} A_{i}$ be a continuous affine function on a building block algebra that is bounded below; $\inf \iota(f)>0$. Then $\inf _{\iota}\left(\phi_{i j *}(f)\right)>$ 0 for all $j>i$.

Proof. From the structure theory of representations of building block algebras $A_{i}\left(C_{i}, D_{i}, b_{i}\right)$, we know that for any homomorphism $\phi_{i j}$ and $y \in[0,1]$ there exist positive integers $M_{j, y}$ and $E_{j, y}$, and real numbers $x_{k} \in(0,1), k=$ $1, \ldots, M_{j, y}$ such that $\phi_{i j}(y)\left(f^{\prime}\right)$ is unitarily equivalent to

$$
\bigoplus_{k=1}^{M_{j, y}} f^{\prime}\left(x_{k}\right) \bigoplus_{1}^{E_{j, y}} e_{\infty}\left(f^{\prime}\right) \bigoplus_{1}^{Z_{j, y}} 0
$$

for all $f^{\prime} \in A_{i}$. Therefore for $y \in[0,1]$,

$$
\begin{aligned}
\iota\left(\phi_{i j *}(f)\right)(y) & =\operatorname{tr}_{D_{j}}\left(\phi_{i j}\left(f^{\prime}\right)(y)\right) \\
& =\frac{\operatorname{dim}\left(D_{i}\right)}{\operatorname{dim}\left(D_{j}\right)} \sum_{k=1}^{M_{j, y}} \operatorname{tr}_{D_{i}}\left(f^{\prime}\left(x_{k}\right)\right)+\frac{\operatorname{dim}\left(C_{i}\right)}{\operatorname{dim}\left(D_{j}\right)} \sum_{k=1}^{E_{j, y}} \operatorname{tr}_{C_{i}}\left(e_{\infty}\left(f^{\prime}\right)\right)
\end{aligned}
$$




$$
\begin{aligned}
& =\frac{\operatorname{dim}\left(D_{i}\right)}{\operatorname{dim}\left(D_{j}\right)} \sum_{k=1}^{M_{j, y}} \iota(f)\left(x_{k}\right)+\frac{\operatorname{dim}\left(C_{i}\right)}{\operatorname{dim}\left(D_{j}\right)} \sum_{k=1}^{E_{j, y}} \iota(f)(1) \\
& >0,
\end{aligned}
$$

where $f^{\prime} \in A_{i}$ is any lift of $f \in \operatorname{Aff} T^{+} A_{i}$ -

As $\inf \iota\left(n_{j}\right)>0$ for all $j \geq 1$, by Proposition 2.0.2, $\Sigma_{n_{j}}$ is compact base for the tracial cone $T^{+} A_{j}$, and therefore induces a bounded norm $\|\cdot\|_{n_{j}}$ on the affine function space $\mathrm{Aff} T^{+} A_{j}$.

Proposition 2.1.2 Let $n_{1} \in \operatorname{Aff} T^{+} A_{1}$ be such that $\iota\left(n_{1}\right)>0$, and let $n_{j}=$ $\phi_{1 j *}\left(n_{1}\right)$ for all $j>1$. Then $\phi_{i j}^{*}\left(\Sigma_{n_{j}}\right) \subset \Sigma_{n_{i}}$

Proof. Consider the following calculation:

$\Sigma_{n_{j}}=\Sigma_{\phi_{i j *}\left(n_{i}\right)}=\left\{\tau \in T^{+} A_{j}: \phi_{i j \star}\left(n_{i}\right)(\tau)=1\right\}=\left\{\tau \in T^{+} A_{j}: n_{i}\left(\phi_{i j}^{*}(\tau)\right)=1\right\}$.

It follows that

$$
\phi_{i j}^{*}\left(\Sigma_{n_{j}}\right) \subset \Sigma_{n_{i}}
$$

Corollary 2.1.1 Let $n_{1} \in \operatorname{Aff} T^{+} A_{1}$ be an affine function such that inf $\iota\left(n_{1}\right)>$ 0 , and let $n_{j}=\phi_{1 j *}\left(n_{1}\right)$ for all $j>1$. Then $\inf \iota_{n_{i}}^{\prime}(f) \leq \inf \iota_{n_{j}}^{\prime}\left(\phi_{i j *}(f)\right)$ for any affine function $f \in \operatorname{Aff} T^{+} A_{i}$ if $j \geq i$.

Proof. By Proposition 2.1.2, $\phi_{i j}^{*}\left(\Sigma_{n_{j}}\right) \subset \Sigma_{n_{i^{*}}}$ Therefore, for $j \geq i$,

$$
\begin{aligned}
\inf \iota_{n_{j}}^{\prime}\left(\phi_{i j *}(f)\right) & =\inf \left\{\phi_{i j *}(f)(\tau): \tau \in \Sigma_{n_{j}}\right\} \\
& =\inf \left\{f(\tau): \tau \in \phi_{i j}^{*}\left(\Sigma_{n_{j}}\right)\right\}
\end{aligned}
$$




$$
\begin{aligned}
& \geq \inf \left\{f(\tau): \tau \in \Sigma_{n_{i}}\right\} \\
& =\inf \iota_{n_{i}}^{\prime}(f) .
\end{aligned}
$$

By Proposition 2.1.2, $\Sigma_{\pi_{i}}$ forms a coherent sequence of sets for the inverse limit system $\left(T^{+} A_{i}, \phi_{i j}^{*}\right)$. As $n_{1}$ is in the Pedersen ideal of the simple inductive limit algebra $A, \infty>\tau\left(n_{1}\right)>0$ for all $\tau \in T^{+} A$. Therefore the tracial cone $T^{+} A$ is generated by positive multiples of threads of the restricted inverse limit system

$$
\left(\Sigma_{n_{i},} \phi_{i j}^{*}\right)
$$

Let the set of threads of this inverse system be denoted by $\Sigma_{n_{\infty}}$. Since each of the finite stages $\Sigma_{n_{i}}$ is compact, so is the set of threads. Therefore $\Sigma_{n_{\infty}}$ is a compact base for the cone of traces of the inductive limit algebra $A$ and hence induces a norm on its space of continuous affine functions. Another way to define $\Sigma_{n_{\infty}}$ is as the subset of $T^{+} A$ of traces that are equal to one on the element $n_{\infty} \in \mathrm{Aff} T^{+} A$. Recall from Proposition 2.0.3 that $\inf \iota_{n_{i}}^{\prime}(f)=\inf \left\{f(\tau): \tau \in \Sigma_{n_{i}}\right\}$; using this definition it is easy to check the following continuity properties for the infimum and supremum:

$$
\begin{aligned}
\inf \left\{\phi_{i \infty *}(f)(\tau): \tau \in \Sigma_{n_{\infty}}\right\} & =\lim _{j \rightarrow \infty} \inf \iota_{n_{j}}^{\prime}\left(\phi_{i j *}(f)\right) \\
\sup \left\{\phi_{i \infty *}(f)(\tau): \tau \in \Sigma_{n_{\infty}}\right\} & =\lim _{j \rightarrow \infty} \sup \iota_{n_{j}}^{\prime}\left(\phi_{i j *}(f)\right)
\end{aligned}
$$

for all $f \in \operatorname{Aff} T^{+} A_{i}$. In particular it should be noted that $\Sigma_{n_{\infty}}$ does not contain the zero trace; this follows immediately from the fact that every trace in $\Sigma_{n_{\infty}}$ evaluates to one on the element $n_{\infty} \in \mathrm{Aff} T^{+} A$.

To apply Elliott's intertwining argument it is necessary to factor the invariant at each finite stage through a finite space, approximately on finitely 
many elements:

$$
\left(\operatorname{Aff} T^{+} A_{i},\|\cdot\|_{A_{i}, n_{i}}\right) \rightarrow\left(C\left(\mathbb{R}_{+}^{N}\right),\|\cdot\|_{A_{i}, n_{i}}\right) \rightarrow\left(\operatorname{Aff} T^{+} A_{i},\|\cdot\|_{A_{i}, n_{i}}\right)
$$

Given a finite set $F \subset$ Aff $T^{+} A_{i}$, this is accomplished as in [Ell1] by choosing a sufficiently fine partition of unity $\left\{\gamma_{i}\right\}_{1}^{N} \subset \mathrm{Aff} T^{+} A_{i}$ and then approximately factorizing the identity map as follows:

$$
f \mapsto\left(f\left(x_{1}\right), f\left(x_{2}\right), f\left(x_{3}\right), \cdots, f\left(x_{N}\right)\right) \mapsto \sum_{i=1}^{N} f\left(x_{i}\right) \gamma_{i}
$$

Recall that in Proposition 2.0.3 we established the isomorphism $\iota_{n_{i}}^{\prime}$ : $\left(\right.$ AffT $\left.T^{+} A_{i},\|\cdot\|_{n_{i}}\right) \rightarrow\left(C(\mathbb{T}),\|\cdot\|_{\infty}\right)$. Given a finite set $\left\{x_{k}\right\}_{k=1}^{N} \subset[0,1)$ and $n_{i} \in \mathrm{Aff} T^{+} A_{i}$ such that $\inf \iota\left(n_{i}\right)>0$ define a positive map $\rho^{\prime}$ as follows:

$$
\begin{aligned}
\rho^{\prime}: \operatorname{Aff}^{+} A_{i} & \rightarrow \mathbb{R}_{+}^{N} \\
f & \mapsto \iota_{n_{i}}^{\prime}(f)\left(x_{k}\right), k=1, \ldots, N .
\end{aligned}
$$

Define the norms $\|\cdot\|_{A_{i}}$ and $\|\cdot\|_{n_{i}}$ on $\left(u_{k}\right)_{k=1}^{N} \in \mathbb{R}_{+}^{N}$ as follows:

$$
\begin{aligned}
& \left\|\left(u_{k}\right)_{1}^{N}\right\|_{n_{i}}=\sup \left\{u_{k}\right\}_{k=1}^{N} \\
& \left\|\left(u_{k}\right)_{1}^{N}\right\|_{A_{i}}=\sup \left\{u_{k}\left(\iota\left(n_{k}\right)\left(x_{k}\right)\right)\right\}_{k=1-}^{N}
\end{aligned}
$$

It is clear that $\rho^{\prime}$ is contractive with respect to the norm $\|\cdot\|_{n_{i^{-}}}$Let us check that the map is also contractive with respect to the norm $\|\cdot\| A_{A_{i}} ;$ if $f \in \mathrm{Aff} T^{+} A_{i}$ then

$$
\begin{aligned}
\left\|\rho^{\prime}(f)\right\|_{A_{i}} & =\sup \left\{\iota_{n_{i}}^{\prime}(f)\left(x_{k}\right) \iota\left(n_{i}\right)\left(x_{k}\right)\right\}_{k=1}^{N} \\
& =\sup \left\{\iota(f)\left(x_{k}\right)\right\}_{k=1}^{N} \\
& \leq\|f\|_{A_{i}} .
\end{aligned}
$$


Lemma 2.1.1 Let $A(C, D, a)$ be a building block algebra and $n \in$ Aff $T^{+} A$ such that $\inf \iota(n)>0$ and $\|n\|_{A} \leq 1$. For any $\varepsilon>0$ and finite set $F \subset$ (Aff $T^{+} A,\|\cdot\|_{A, n}$ ) there exists a positive integer $N$, finite sets $\left\{x_{k}\right\}_{k=1}^{N} \subset[0,1)$ and $\left\{\gamma_{k}\right\}_{k=1}^{N} \subset \operatorname{Aff} T^{+} A$, and a positive contraction $\rho \equiv\left(\rho_{k}\right)_{k=1}^{N}=\left(\operatorname{Aff} T^{+} A, \|\right.$. $\left.\|_{A, n}\right) \rightarrow\left(\mathbb{R}_{+}^{N},\|\cdot\|_{A, n}\right)$ such that

$$
\left\|f-\sum_{k=1}^{N} \rho_{k}(f) \gamma_{k}\right\|_{A, n}<2 \varepsilon
$$

for all $f \in F$ and

$$
\left\|\sum_{k=1}^{N} \rho_{k}(f) \gamma_{k}\right\|_{A, n} \leq\|f\|_{A, n}
$$

for all $f \in \mathrm{Aff}^{+} \boldsymbol{A}$.

Proof. By choosing an appropriate value of $\varepsilon$ in the hypothesis of the lemma, we can reduce to the case that $\|f\|_{A, n} \leq 1$. Let $\left\{\gamma_{k}^{\prime}\right\}_{k=1}^{N} \subset C(\mathbb{T})$ be a partition of unity, and let $\left\{x_{k}\right\}_{k=1}^{N} \subset[0,1)$ be such that

$$
\left\|\iota_{n}^{\prime}(f)-\sum_{k=1}^{N} \iota_{n}^{\prime}(f)\left(x_{k}\right) \gamma_{k}^{\prime}\right\|_{\infty}<\varepsilon
$$

for all $f \in F$ and

$$
\left\|\frac{\iota(n)(x)}{\iota(n)\left(x_{k}\right)}-1\right\|_{\infty}<\varepsilon \text { for } x \in \operatorname{supp}\left(\gamma_{k}^{\prime}\right)
$$

(We have used $\operatorname{supp}\left(\gamma_{k}^{\prime}\right)$ to denote the subset $\left\{t \in \mathbb{T}: \gamma_{k}^{\prime}(t)>0\right\}$.) Let $\rho^{\prime}$ be defined as in equation (2.8) with respect to the finite set $\left\{x_{k}\right\}_{k=1}^{N}$, and $n \in \operatorname{Aff}^{+} A$. Let $\rho(f)=\frac{1}{1+\varepsilon} \rho^{\prime}(f)=\frac{1}{1+\varepsilon}\left(\iota_{n}^{\prime}(f)\left(x_{k}\right)\right)_{k=1}^{N}$ and $\gamma_{k}=\left(\iota_{n}^{\prime}\right)^{-1}\left(\gamma_{k}^{\prime}\right)$. Then

$$
\left\|f-\sum_{k=1}^{N} \rho_{k}(f) \gamma_{k}\right\|_{n}
$$




$$
\begin{aligned}
& =\left\|\iota_{n}^{\prime}(f)-\frac{1}{1+\varepsilon} \sum_{k=1}^{N} \iota_{n}^{\prime}(f)\left(x_{k}\right) \gamma_{k}^{\prime}\right\|_{\infty} \\
& \leq\left\|\iota_{n}^{\prime}(f)-\sum_{k=1}^{N} \iota_{n}^{\prime}(f)\left(x_{k}\right) \gamma_{k}^{\prime}\right\|_{\infty}+\left\|\sum_{k=1}^{N} \iota_{n}^{\prime}(f)\left(x_{k}\right) \gamma_{k}^{\prime}-\frac{1}{1+\varepsilon} \sum_{k=1}^{N} \iota_{n}^{\prime}(f)\left(x_{k}\right) \gamma_{k}^{\prime}\right\|_{\infty} \\
& \leq \varepsilon+\frac{\varepsilon}{1+\varepsilon}\left\|\iota_{n}^{\prime}(f)\right\|_{\infty} \\
& \leq 2 \varepsilon .
\end{aligned}
$$

And, using the above inequality,

$$
\begin{aligned}
\left\|f-\sum_{k=1}^{N} \rho_{k}(f) \gamma_{k}\right\|_{A} & =\left\|\iota(f)-\sum_{k=1}^{N} \rho_{k}(f) \iota\left(\gamma_{k}\right)\right\|_{\infty} \\
& =\left\|\left(\iota_{n}^{\prime}(f)-\frac{1}{1+\varepsilon} \sum_{k=1}^{N} \iota_{n}^{\prime}(f)\left(x_{k}\right) \gamma_{k}^{\prime}\right) \iota(n)\right\|_{\infty} \\
& \leq 2 \varepsilon\|n\|_{A} \leq 2 \varepsilon .
\end{aligned}
$$

To complete the proof of the lemma we need to check that the map $f \mapsto$ $\sum_{k=1}^{N} \rho_{k}(f) \gamma_{k}$ is approximately contractive with respect to both norms:

$$
\begin{aligned}
\left\|\sum_{k=1}^{N} \rho_{k}(f) \gamma_{k}\right\|_{n} & =\left\|\frac{1}{1+\varepsilon} \sum_{k=1}^{N} \iota_{n}^{\prime}(f)\left(x_{k}\right) \gamma_{k}^{\prime}\right\|_{\infty} \\
& \leq\left\|\iota_{n}^{\prime}(f)\right\|_{\infty} \leq\|f\|_{n} .
\end{aligned}
$$

With respect to the norm $\|\cdot\|_{A}$ we can make a similar estimate as follows:

$$
\begin{aligned}
\left\|\sum_{k=1}^{N} \rho_{k}(f) \gamma_{k}\right\|_{A} & =\left\|\frac{1}{1+\varepsilon} \sum_{k=1}^{N} \iota_{n}^{\prime}(f)\left(x_{k}\right) \iota\left(\gamma_{k}\right)\right\|_{\infty} \\
& =\frac{1}{1+\varepsilon}\left\|\sum_{k=1}^{N} \frac{\iota(f)\left(x_{k}\right)}{\iota(n)\left(x_{k}\right)} \iota(n) \gamma_{k}^{\prime}\right\|_{\infty} \\
& =\frac{1}{1+\varepsilon}\left\|\sum_{k=1}^{N} \iota(f)\left(x_{k}\right) \gamma_{k}^{\prime} \frac{\iota(n)}{\iota(n)\left(x_{k}\right)}\right\|_{\infty}
\end{aligned}
$$


By assumption, the partition of unity was chosen fine enough so that $\| \frac{\iota(n)(x)}{\iota(\pi)\left(x_{k}\right)}-$ $1 \|_{\infty}<\varepsilon$ for $x \in \operatorname{supp}\left(\gamma_{k}^{\prime}\right)$. Therefore

$$
\left\|\frac{\iota(n)(x)}{\iota(n)\left(x_{k}\right)}\right\|_{\infty}<1+\varepsilon \text { for } x \in \operatorname{supp}\left(\gamma_{k}^{\prime}\right)
$$

and hence

$$
\begin{aligned}
\left\|\sum_{k=1}^{N} \rho_{k}(f) \gamma_{k}\right\|_{A} & =\frac{1}{1+\varepsilon}\left\|\sum_{k=1}^{N} \iota(f)\left(x_{k}\right) \gamma_{k}^{\prime} \frac{\iota(n)}{\iota(n)\left(x_{k}\right)}\right\|_{\infty} \\
& \leq \frac{1}{1+\varepsilon}(1+\varepsilon)\left\|\sum_{k=1}^{N} \iota(f)\left(x_{k}\right) \gamma_{k}^{\prime}\right\|_{\infty} \\
& \leq\|\iota(f)\|_{\infty}=\|f\|_{A} .
\end{aligned}
$$

This completes the proof of the lemma.

Let $n_{1} \in \operatorname{Aff} T^{+} A_{1}$ and $m_{1} \in \operatorname{Aff} T^{+} B_{1}$ be affine functions such that

$$
\inf \left(\iota\left(n_{1}\right)\right)>0 \text { and } \inf \left(\iota\left(m_{1}\right)\right)>0
$$

Let

$$
n_{i}=\phi_{1 i *}\left(n_{1}\right) \text { and } m_{i}=\phi_{1 i *}\left(m_{1}\right)
$$

The remaining part of this section will be devoted to constructing an intertwining of the affine function spaces $\lim _{\longrightarrow}\left(\right.$ Aff $\left.T^{+} A_{i},\|\cdot\|_{A_{i}, n_{i}}\right)$ and $\lim _{\rightarrow}\left(\right.$ Aff $T^{+} B_{i}, \| \cdot$ $\left.\|_{B_{i}, m_{i}}\right)$. The inclusion of Propositions 2.1.3 and 2.1.4 at the end of this section sets the stage for the intertwining of the inductive limit algebras $\lim _{\longrightarrow}\left(A_{i}, \phi_{i j}\right)$ and $\lim \left(B_{i}, \psi_{i j}\right)$ which will be carried out in Section 2.2.

By Lemma 2.1.1, given a finite set $F \subset \operatorname{Aff} T^{+} A_{i}$ and $\varepsilon>0$ there exists an positive integer $N\left(A_{i}\right)$ and an approximate factorization of the 
identity map on (Aff $T+A_{i},\|\cdot\|_{A_{i}, n_{i}}$ ) through the finitely generated cone $\left(\mathbb{R}_{+}^{N\left(A_{i}\right)},\|\cdot\|_{A_{i}, n_{i}}\right)$ within $\varepsilon$ on the finite set $F$. By choosing an appropriate dense sequence in $\mathrm{Aff}^{+} A$ and factorizing the identity map on finite subsets of the sequence at each finite stage within an appropriate tolerance we can construct an approximate intertwining of $\lim \left(\mathrm{Aff} T^{+} A_{i},\|\cdot\|_{A_{i}, n_{i}}\right)$ and $\lim _{\rightarrow}\left(\mathbb{R}_{+}^{N\left(A_{i}\right)},\|\cdot\|_{A_{i}, n_{i}}\right)$. It then follows directly from Theorem 2.2 of [E112] that $\lim _{\longrightarrow}\left(\operatorname{Aff} T^{+} A_{i},\|\cdot\|_{A_{i}, n_{i}}\right)$ and $\lim \longrightarrow\left(\mathbb{R}_{+}^{N\left(A_{i}\right)},\|\cdot\|_{A_{i}, n_{i}}\right)$ are isomorphic. Similarly we can construct an approximate intertwining of $\lim \left(A\right.$ Aff $\left.T^{+} B_{i},\|\cdot\|_{B_{i}, m_{i}}\right)$ and $\lim _{\longrightarrow}\left(\mathbb{R}_{+}^{N\left(B_{i}\right)},\|\cdot\|_{B_{i}, m_{i}}\right)$.

By the above arguments, and the assumption that $\left(A f f T^{+} A,\|\cdot\|_{A}\right)$ is isomorphic to $\left(\right.$ Aff $\left.T^{+} B,\|\cdot\|_{B}\right)$, we know that $\lim _{\longrightarrow}\left(\mathbb{R}_{+}^{N\left(A_{i}\right)},\|\cdot\|_{A_{i}}\right)$ is isomorphic to $\lim _{\longrightarrow}\left(\mathbb{R}_{+}^{N\left(B_{i}\right)},\|\cdot\|_{B_{i}}\right)$. It is easy to intertwine finitely generated normed spaces; the argument is very similar to that given in [Ell1], and appears explicitly in [Ell3]. The argument in our case is exactly the same, except that the given isomorphism, $\phi: \mathrm{Aff} T^{+} A \rightarrow \mathrm{Aff} T^{+} B$ is not isometric with respect to the norms $\|\cdot\|_{n_{\infty}}$ and $\|\cdot\|_{m_{\infty}}$. It is, however, for the purposes of constructing an intertwining enough to check that the norms are equivalent with respect to the isomorphism $\phi$ in the following sense: there exist positive constants $M_{1}$ and $M_{2}$ such that

$$
M_{2}\|f\|_{n_{\infty}} \leq\|\phi(f)\|_{m_{\infty}} \leq M_{1}\|f\|_{n_{\infty}}
$$

for all $f \in \operatorname{Aff} T^{+} A$. Let $M_{1}=\left\|\phi\left(n_{\infty}\right)\right\|_{m_{\infty}}=\sup \left\{\phi\left(n_{\infty}\right)(\tau): \tau \in \Sigma_{m_{\infty}}\right\}$. Using this expression for the norm, it follows that

$$
\begin{aligned}
\|\phi(f)\|_{m_{\infty}} & =\sup \left\{f\left(\phi^{*}(\tau)\right): \tau \in \Sigma_{m_{\infty}}\right\} \\
& \leq \sup \left\{f\left(\phi^{*}(\tau)\right): \phi\left(n_{\infty}\right)(\tau)=M_{1}\right\}
\end{aligned}
$$




$$
\begin{aligned}
& =M_{1} \sup \left\{f\left(\phi^{*}(\tau)\right): \phi\left(n_{\infty}\right)(\tau)=1\right\} \\
& =M_{1} \sup \left\{f\left(\phi^{*}(\tau)\right): n_{\infty}\left(\left(\phi^{*}(\tau)\right)=1\right\}\right. \\
& =M_{1} \sup \|f\|_{n_{\infty}}
\end{aligned}
$$

for all $f \in \operatorname{Aff} T^{+} A$. Similarly, $\left\|\phi^{-1}(f)\right\|_{n_{\infty}} \leq M_{1}^{\prime}\|f\|_{m_{\infty}}$ for all $f \in \operatorname{Aff} T^{+} B$. Let $M_{2}=1 / M_{1}^{\prime}$, then together with the previous result

$$
M_{2}\|f\|_{n_{\infty}} \leq\|\phi(f)\|_{m_{\infty}} \leq M_{1}\|f\|_{n_{\infty}}
$$

for all $f \in \operatorname{Aff} T^{+} A$.

We can also prove that there exist real numbers $m_{1}, m_{2}>0$ such that

$$
\inf \left\{\phi(f)(\tau): \tau \in \Sigma_{m_{\infty}}\right\} \geq m_{1} \inf \left\{f(\tau): \tau \in \Sigma_{n_{\infty}}\right\}
$$

and

$$
\inf \left\{\phi(f)(\tau): \tau \in \Sigma_{m_{\infty}}\right\} \leq m_{2} \inf \left\{f(\tau): \tau \in \Sigma_{n_{\infty}}\right\}
$$

by similar means. Let $m_{1}=\inf \left\{\left(\phi\left(n_{\infty}\right)\right)(\tau): \tau \in \Sigma_{m_{\infty}}\right\}$ (these estimates will prove useful at a later stage in the intertwining argument). Then

$$
\begin{aligned}
\inf \left\{\phi(f)(\tau): \tau \in \Sigma_{m_{\infty}}\right\} & \geq \inf \left\{f\left(\phi^{*}(\tau)\right): \phi\left(n_{\infty}\right)(\tau)=m_{1}\right\} \\
& =m_{1} \inf \left\{f\left(\phi^{*}(\tau)\right): \phi\left(n_{\infty}\right)(\tau)=1\right\} \\
& =m_{1} \inf \left\{f\left(\phi^{*}(\tau)\right): n_{\infty}\left(\phi^{*}(\tau)\right)=1\right\} \\
& =m_{1} \inf \left\{f(\tau): n_{\infty}(\tau)=1\right\} \\
& =m_{1} \inf \left\{f(\tau): \tau \in \Sigma_{n_{\infty}}\right\}
\end{aligned}
$$

The identical statement that holds for $\phi^{-1}$ can be used to find the constant $m_{2}$. 
Approximate intertwinings are transitive (this property is used implicitly in constructing an intertwining of the affine function spaces in [EL3]), and by the arguments given previously, we have therefore constructed an approximate intertwining of $\left(\left(\operatorname{Aff} T^{+} A_{i},\|-\|_{A_{i}, n_{i}}\right), \phi_{i j *}\right)$ and ((Aff $T^{+} B_{i}, \| \cdot$ $\left.\left.\|_{B_{i}, m_{i}}\right), \psi_{i j *}\right)$;

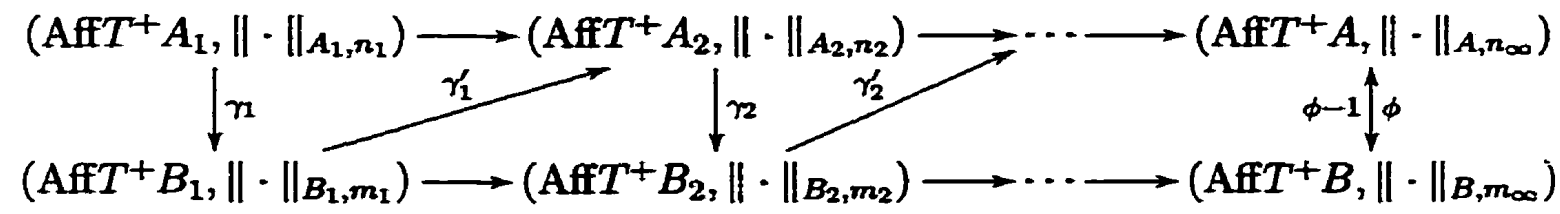

The next step in Elliott's isomorphism algorithm is to lift each of the maps at finite stages between affine function spaces to maps between the building block algebras. This will be accomplished by means of Theorem 3.0.1, the existence theorem. In our setting we cannot apply the existence theorem immediately to the approximately commuting diagram given above. In order to satisfy the hypothesis of the theorem we need to pass to suitable subsequences. Furthermore, the conclusions of the existence theorem do not, as given, preserve the approximate commutativity of the diagram of affine function spaces; the estimate in the conclusion of the theorem is with respect to a norm that is different than the norms given in the approximate intertwining. Both of these difficulties can be surmounted by passing to suitable subsequences in the approximate intertwining, as will be demonstrated in Section 2.2. The following propositions are sufficient to carry out this procedure.

Proposition 2.1.3 At any finite stage $A_{i}$, there exist real numbers $\mu, M>0$ and a positive integer $N$ such that for any affine function $s \in \operatorname{Aff} T^{+} A_{i}$, 
$\|s\|_{A_{i}} \leq 1$ and $\inf (\iota(s))>1 / 2$, and any map $\xi: A_{i} \rightarrow B_{j}$ composed of at least $N$ horizontal steps before a diagonal step, $\|\xi(s)\|_{m_{j}} \leq M$ and $\inf \left(\frac{\iota(\xi(s))}{\iota\left(m_{j}\right)}\right) \geq \mu$.

Proof. The first step to proving the proposition is to find bounds for the norm and infimum of the element $\phi_{i \infty * *}(s)$ in the inductive limit affine function space using the bounds at the finite stage $\mathrm{Aff} T^{+} A_{i}$ given in the hypothesis of the proposition. As $\|\cdot\|_{A_{i}}$ and $\|\cdot\|_{n_{i}}$ are equivalent norms, there exists a positive real number $M^{\prime}>0$ such that $\|s\|_{n_{i}} \leq M^{\prime}$ (where $i$ is fixed) for all $s \in \operatorname{Aff} T^{+} A_{i}$ such that $\|s\|_{A_{i}} \leq 1$. As the connecting maps $\phi_{i j *}$ are contractive, by the equivalence of the norms $\|\cdot\|_{n_{\infty}}$ and $\|\cdot\|_{m_{\infty}}$ given by equation (2.9), there exists a positive constant $M^{\prime \prime}>0$ such that

$$
\left\|\phi \circ \phi_{i \infty *}(s)\right\|_{m_{\infty}} \leq M^{\prime \prime}
$$

for all $s \in \operatorname{Aff} T^{+} A_{i}$ such that $\|s\|_{A_{i}} \leq 1$.

$$
\text { As }\left\|n_{i}\right\|_{A_{i}}=\left\|\iota\left(n_{i}\right)\right\|_{\infty} \leq 1 \text {, }
$$

$$
\begin{aligned}
\inf \left(\iota_{n_{i}}(s)\right) & =\inf \left(\frac{\iota(s)}{\iota\left(n_{i}\right)}\right) \\
& \geq \inf (\iota(s)) \\
& >1 / 2 .
\end{aligned}
$$

By Corollary 2.1.1, the maps $\phi_{i j *}$ are infimum non-decreasing with respect to the element $n_{i}$, and therefore $\inf \left\{\phi_{i \infty *}(s)(\tau): \tau \in \Sigma_{n_{\infty}}\right\}>1 / 2$. Therefore by equation (2.10) there exists a positive constant $m^{\prime}$ such that

$$
\inf \left\{\phi \circ \phi_{i \infty *}(s)(\tau): \tau \in \Sigma_{m_{\infty}}\right\}>m^{\prime}
$$


As the diagram is approximately commuting, there exists a positive integer $N^{\prime}$ such that

$$
\left\|\phi \circ \phi_{i \infty *}(s)-\psi_{k \infty 0_{*}} \circ \gamma_{k} \circ \phi_{i k *}(s)\right\|_{m_{\infty}}<m^{\prime} / 4
$$

for all $k>N^{\prime}$. As the connecting maps $\psi_{i j *}$ are continuous with respect to the sequence of norms and infimums induced by the element $m_{i}$ (see equations (2.5) and (2.6)), and the diagram commutes approximately, there exists a positive integer $N \geq N^{\prime}$ such that

$$
\begin{aligned}
\left|\left\|\gamma_{k} \circ \phi_{i k *}(s)\right\|_{m_{k}}-\left\|\psi_{k \infty * *} \circ \gamma_{k} \circ \phi_{i k *}(s)\right\|_{m_{\infty}}\right| & <m^{\prime} / 4 \\
\left|\inf \left(\left.\gamma_{k} \circ \phi_{i k *}(s)\right|_{\Sigma_{m_{k}}}\right)-\inf \left(\psi_{k \infty \infty *} \circ \gamma_{k} \circ \phi_{i k *}(s) \mid \Sigma_{m_{\infty}}\right)\right| & <m^{\prime} / 4
\end{aligned}
$$

for all $k>N$.

From equation (2.11) and equation (2.14) it follows that

$$
\left\|\psi_{k \infty 0_{*}} \circ \gamma_{k} \circ \phi_{i k *}(s)\right\|_{m_{\infty}} \leq M^{\prime \prime}+m^{\prime} / 4
$$

The above equation together with the estimate in equation (2.15) yields

$$
\left\|\gamma_{k} \circ \phi_{i k *}(s)\right\|_{m_{k}} \leq M^{\prime \prime}+m^{\prime} / 4+m^{\prime} 4=M^{\prime r}+m^{\prime} / 2
$$

Let $M=M^{\prime \prime}+m^{\prime} / 2$, then

$$
\|\xi(s)\|_{m_{k}} \leq M
$$

where $\xi=\gamma_{k} \circ \phi_{i k *}$ is any map from $A_{i}$ to $B_{j}$ composed of at least $N$ horizontal steps before a vertical step.

If we notice that equation (2.14) is equivalent to the statement $\sup \{\phi \circ$ $\left.\phi_{i \infty *}(s)(\tau)-\psi_{k \infty *} \circ \gamma_{k} \circ \phi_{i k *}(s)(\tau): \tau \in \Sigma_{m_{\infty}}\right\}$ then it is apparent that using 
the lower bound on the infimum of $\left.\phi \circ \phi_{i \infty}(s)\right|_{\Sigma_{m_{\infty}}}$ given in equation (2.13) yields a lower bound of $m^{\prime}-m^{\prime} / 4$ for the infimum of $\left.\psi_{k \infty \neq *} \circ \gamma_{k} \circ \phi_{i k *}(s)\right|_{\Sigma_{m_{\infty}}}$ :

$$
\inf \left\{\left.\psi_{k \infty *} \circ \gamma_{k} \circ \phi_{i k *}(s)\right|_{\Sigma_{m \infty}}\right\}>\frac{3}{4} m^{\prime}
$$

The above equation together with the estimate in equation (2.16) yields

$$
\inf \left(\gamma_{k} \circ \phi_{i k *}(s)\left[\Sigma_{m_{k}}\right)>\frac{3}{4} m^{\prime}-\frac{m^{\prime}}{4}\right.
$$

Let $\mu=m^{\prime} / 2$, then

$$
\inf \left(\frac{\iota(\xi(s))}{\iota\left(m_{k}\right)}\right)=\inf \left(\gamma_{k} \circ \phi_{i k *}(s) \mid \Sigma_{m_{k}}\right)>\mu
$$

The following proposition is a consequence of the so-called delta density condition for simple inductive limits.

Proposition 2.1.4 Let $B=\lim _{\rightarrow}\left(B_{i}\left(C_{i}, D_{i}, b_{i}\right), \phi_{i j}\right)$ be a simple inductive limit of building block algebras. Let $m_{1} \in \operatorname{Aff} T^{+} B_{1}$ such that $\inf \left(\iota\left(m_{1}\right)\right)>0$ and let $m_{i}=\phi_{1 i *}\left(m_{1}\right)$. Then for any real number $M>0$, there exists a positive integer $N$ such that for all $j \geq N, \operatorname{dim}\left(D_{j}\right)\left(\iota\left(m_{j}\right)(y)\right)>M$ for all $y \in[0,1]$ and $\frac{\operatorname{dim}\left(D_{j}\right)}{b_{j}}\left(\iota\left(m_{j}\right)(0)\right)>M$.

Proof. It is well known that simple approximately subhomogeneous algebras with injective connecting maps satisfy the so-called delta density condition: for any partition $\left\{\delta_{n}\right\}_{1}^{K}$ of $C[0,1]_{a_{1}}$ there exists a positive integer $N^{\prime}>0$ such that $\inf \left(\iota \circ \phi_{1 j^{*}} \circ \iota^{-1}\left(\delta_{n}\right)\right)>0$ for all $n$ and $j \geq N^{\prime}$.

From the structure theory of representations of building block algebras $B_{i}\left(C_{i}, D_{i}, b_{i}\right)$, we know that for any homomorphism $\phi_{1 j}$ and $y \in[0,1]$ there 
exist positive integers $M_{j, y}$ and $E_{j, y}$, and real numbers $x_{i} \in(0,1), i=$ $1, \ldots, M_{j, y}$ such that $\phi_{1 j}(y)(f)$ is unitarily equivalent to

$$
\bigoplus_{i=1}^{M_{j, y}} f\left(x_{i}\right) \bigoplus_{1}^{E_{j, y}} e_{\infty}(f) \bigoplus_{1}^{Z_{j, y}} 0
$$

for all $f \in B_{1}$. Therefore for $y \in[0,1]$,

$$
\begin{aligned}
\iota\left(\phi_{1 j *}\left(m_{1}\right)\right)(y) & =\operatorname{tr}_{D_{j}}\left(\phi_{1 j}\left(m_{1}^{r}\right)(y)\right) \\
& =\frac{\operatorname{dim}\left(D_{1}\right)}{\operatorname{dim}\left(D_{j}\right)} \sum_{i=1}^{M_{j, y}} \operatorname{tr}_{D_{1}}\left(m_{1}\left(x_{i}\right)\right)+\frac{\operatorname{dim}\left(C_{1}\right)}{\operatorname{dim}\left(D_{j}\right)} \sum_{i=1}^{E_{j, y}} \operatorname{tr}_{C_{1}}\left(e_{\infty}\left(m_{1}\right)\right) \\
& =\frac{\operatorname{dim}\left(D_{1}\right)}{\operatorname{dim}\left(D_{j}\right)} \sum_{i=1}^{M_{j, y}} \iota\left(m_{1}\right)\left(x_{i}\right)+\frac{\operatorname{dim}\left(C_{1}\right)}{\operatorname{dim}\left(D_{j}\right)} \sum_{i=1}^{E_{j, y}} \iota\left(m_{1}\right)(1)
\end{aligned}
$$

where $m_{1}^{\prime} \in A_{1}$ is any lift of $m_{1}$, and $\operatorname{tr}_{D_{j}}$ and $\operatorname{tr}_{C_{i}}$ are the normalized matrix traces on $D_{j}$ and $C_{i}$ respectively. For $y=0$ we can make a stronger statement; recall that all irreducible representations of $A_{1}$ have multiplicity $b_{j}$ at the left endpoint. Therefore $\phi_{1 j}(0)(f)$ is unitarily equivalent to

$$
\bigoplus_{1}^{b_{j}}\left(\bigoplus_{1}^{M_{j, 0}^{\prime}} f\left(x_{i}\right) \bigoplus_{1}^{E_{j, 0}^{\prime}} e_{\infty}(f) \bigoplus_{1}^{Z_{j, 0}^{\prime}} 0\right)
$$

where $b_{j} M_{j, 0}^{\prime}=M_{j, 0}, b_{j} E_{j, 0}^{\prime}=E_{j, 0}$ and $b_{j} Z_{j, 0}^{\prime}=Z_{j, 0}$. Therefore, by an argument similar to that given by equation (2.18),

$$
\begin{aligned}
\iota\left(\phi_{1 j *}\left(m_{1}\right)\right)(0)= & \frac{b_{j}}{b_{j}+1}\left(\frac{\operatorname{dim}\left(D_{1}\right)}{\operatorname{dim}\left(C_{j}\right)} \sum_{i=1}^{M_{j, 0}^{\prime}} \iota\left(m_{1}\right)\left(x_{i}\right)\right. \\
& +\frac{\operatorname{dim}\left(C_{1}\right)}{\operatorname{dim}\left(C_{j}\right)} \sum_{i=1}^{E_{j, 0}^{\prime}} \iota\left(m_{1}\right)(1) .
\end{aligned}
$$

Let $\alpha=\inf \left(c\left(m_{1}\right)\right)$. Then by equation (2.18),

$$
\operatorname{dim}\left(D_{j}\right) \iota\left(\phi_{1 j *}\left(m_{1}\right)\right)(y) \geq M_{j, y} \alpha
$$


and by equation (2.19),

$$
\begin{aligned}
\frac{\operatorname{dim}\left(D_{j}\right)}{b_{j}} \iota\left(\phi_{1 j *}\left(m_{1}\right)\right)(0) & =\operatorname{dim}\left(C_{j}\right) \frac{b_{j}+1}{b_{j}} \iota\left(\phi_{1 j *}\left(m_{1}\right)\right)(0) \\
& \geq M_{j, y}^{\prime} \alpha
\end{aligned}
$$

(we have used the fact that $\operatorname{dim}\left(C_{j}\right)=\left(b_{j}+1\right) \operatorname{dim}\left(D_{j}\right)$ ). It follows from the delta density condition that as $j$ tends to infinity, $\inf \left\{M_{j, y}: y \in[0,1]\right\}$ and $M_{j, 0}$ tend to infinity. As $\alpha$ is fixed this completes the proof of the proposition.

\subsection{Isomorphism}

In this section we will lift the approximate intertwining of the affine function spaces to an approximate intertwining of the algebras. By Elliott's approximate intertwining theorem we can then conclude that the algebras $A$ and $B$ are isomorphic.

Lifting an approximate intertwining of affine function spaces to an approximate (not necessarily commuting) intertwining of the building block algebras is accomplished in three easy steps using Propositions 2.1.3 and 2.1.4 and the existence theorem. We will show that given a finite set $F \subset \mathrm{AffT} T^{+} A_{i}$ and $\varepsilon>0$ there exists a positive integer $L$ such that for any $j \geq L$, $\xi_{j} \equiv \gamma_{j} \circ \phi_{i j *}:\left(\operatorname{Aff} T^{+} A_{i},\|\cdot\|_{n_{i}}\right) \rightarrow\left(\operatorname{Aff} T^{+} B_{j},\|\cdot\|_{m_{j}}\right)$ can be lifted (approximately with respect to the norm $\|\cdot\|_{m_{j}}$ on the finite set $F$ ) to a homomorphism $\psi: A_{i} \rightarrow B_{j}$.

Step 1. By Proposition 2.1.3, there exists real numbers $\mu, M_{1}$ and a positive integer $N_{1}$ such that for any affine function $s \in A f f T^{+} A_{i}$ such that 
$\|s\|_{A_{i}} \leq 1$ and $\inf (c(s))>1 / 2$,

$$
\left\|\xi_{j}(s)\right\|_{m_{j}} \leq M_{1}
$$

and

$$
\inf \left(\frac{\iota\left(\xi_{j}(s)\right)}{\iota\left(m_{j}\right)}\right) \geq \mu
$$

for all $j \geq N_{1}$.

Step 2. We apply the existence theorem to the finite set $F$ and epsilon value of $\varepsilon / M_{1}$ to yield a positive integer $N$ and an affine function $\nu,\|\nu\|_{A_{i}} \leq 1$ and $\inf (\iota(\nu))>1 / 2$ (the affine function $\nu$ is labeled as $n$ in the statement of the existence theorem; we have chosen the label $\nu$ for reasons of clarity in the argument that follows). In particular, for $j \geq N_{1}$, and for the affine function $\nu$, equations (2.20) and (2.21) take the form:

$$
\left\|\xi_{j}(\nu)\right\|_{m_{j}} \leq M_{1}
$$

and

$$
\iota\left(\xi_{j}(\nu)\right)(y) \geq \mu \iota\left(m_{j}\right)(y)
$$

for all $y \in[0,1]$.

Step 3. By Proposition 2.1.4, for the real number $N / \mu$, there is a positive integer $N_{2}$ such that for all $j \geq N_{2}$,

$$
\operatorname{dim}\left(D_{j}\right)\left(\iota\left(m_{j}\right)(y)\right)>N / \mu \text { for all } y \in[0,1]
$$


and

$$
\frac{\operatorname{dim}\left(D_{j}\right)}{b_{j}}\left(\iota\left(m_{j}\right)(0)\right)>N / \mu .
$$

Let $L=\max \left(N_{1}, N_{2}\right)$. For $j \geq L$, by equations (2.23), (2.24) and (2.25),

$$
\begin{aligned}
\operatorname{dim}\left(D_{j}\right)\left(\iota\left(\xi_{j}(\nu)\right)(y)\right) & \geq \mu \operatorname{dim}\left(D_{j}\right)\left(\iota\left(m_{j}\right)(y)\right) \\
& >\mu \frac{N}{\mu}=N
\end{aligned}
$$

and

$$
\begin{aligned}
\frac{\operatorname{dim}\left(D_{j}\right)}{b_{j}}\left(\iota\left(\xi_{j}(\nu)\right)(0)\right) & \geq \mu \frac{\operatorname{dim}\left(D_{j}\right)}{b_{j}}\left(\iota\left(m_{j}\right)(0)\right) \\
& >\mu \frac{N}{\mu}=N .
\end{aligned}
$$

By the above equations, for $j \geq L$ the hypothesis of Theorem 3.0.1, the existence theorem, have been satisfied for the finite set $F, \varepsilon / M_{1}$ and contractive positive map $\xi_{j}: \operatorname{Aff} T^{+} A_{i} \rightarrow \operatorname{Aff} T^{+} B_{j}$. Therefore, we may apply the theorem to conclude that there exists a homomorphism $\psi: A_{i} \rightarrow B_{j}$ with induced map $\psi_{*}: \operatorname{Aff} T^{+} A_{i} \rightarrow \operatorname{Aff} T^{+} B_{j}$ such that

$$
\left\|\xi_{j}(f)-\psi_{*}(f)\right\|_{\xi_{j}(\nu)}<\varepsilon / M_{I}
$$

for $f \in F$. Using equation (2.22) it easy to prove the following estimate on norms:

$$
\|\cdot\|_{m_{j}} \leq M_{1}\|\cdot\|_{\xi_{j}(\nu)}
$$

we can argue as follows

$$
\begin{aligned}
\|f\|_{m_{j}} & =\left\|\frac{\iota(f)}{\iota\left(m_{j}\right.}\right\|_{\infty} \\
& \leq\left\|\frac{\iota(f)}{\iota\left(\xi_{j}(\nu)\right)}\right\|_{\infty}\left\|\frac{\iota\left(\xi_{j}(\nu)\right)}{\iota\left(m_{j}\right.}\right\|_{\infty}
\end{aligned}
$$




$$
\leq M_{1}\|f\|_{\xi_{j}(\nu)}
$$

Therefore, by equation (2.26),

$$
\left\|\xi_{j}(f)-\psi_{*}(f)\right\|_{m_{j}}<\varepsilon
$$

for $f \in F$. This completes the part of the intertwining argument in which maps between the affine function spaces are lifted to maps between the building block algebras.

At this stage we have a (not necessarily approximately commutative) diagram of algebra homomorphisms that induces an approximately commutative diagram of inductive limit systems $\left(\left(\operatorname{Aff} T^{+} A_{i},\|\cdot\|_{A_{i}, n_{i}}\right), \phi_{i j *}\right)$ and $\left(\left(\operatorname{Aff} T^{+} B_{i},\|\cdot\|_{B_{i}, m_{i}}\right), \psi_{i j *}\right)$. We can apply the uniqueness theorem successively to each triangle in the diagram to obtain an approximately commuting diagram. The argument is straight forward. Given a finite set $F \subset A_{i}$ and $\varepsilon>0$ there exists two families of affine functions $\left\{\delta_{k}\right\}_{1}^{n}$ and $\left\{h_{k}\right\}_{1}^{n}$ for which certain conditions have to be met if we are to apply the uniqueness theorem.

As the algebra $A$ is simple and the connecting maps $\phi_{i j}$ are injective, by Corollary 2.1.1 there exists a positive integer $N_{1}$ and a real number $\delta>0$ such that for all $j \geq N_{1}$ the infimum of each element $\delta_{k}$ restricted to the compact base $\Sigma_{n_{j}}$ is bounded below by $\delta$ :

$$
\inf \left(\left.\phi_{i j *}\left(\delta_{k}\right)\right|_{\Sigma_{n_{j}}}\right)>\delta \text { for } k=1, \ldots, n
$$

As the diagram of affine function spaces commutes approximately, there exists a positive integer $N_{2}$ such that for all $j \geq N_{2}$,

$$
\left.\left(\gamma_{l}^{\prime} \circ \psi_{l j *} \circ \gamma_{j} \circ \phi_{i j *}\left(h_{k}\right)-\phi_{i l *}\left(h_{k}\right)\right)\right|_{\Sigma_{n_{l}}}<\delta / 2
$$


for $k=1, \ldots, n$. Therefore, by the equations above, for $j \geq \max \left(N_{1}, N_{2}\right)$ and for any two diagonal maps between building block algebras, $\xi_{j}: A_{i} \rightarrow B_{j}$ and $\xi_{l}^{\prime}=B_{j} \rightarrow A_{l}$, by Theorem 4.0.1, the uniqueness theorem, there exists a unitary $U$ in the unitization of $A_{l}$ such that

$$
\left\|\phi_{i t}(f)-\operatorname{Ad} U\left(\xi_{l}^{\prime} \circ \xi_{j}(f)\right)\right\|<\varepsilon
$$

for $f \in F$. This completes the approximate intertwining. By Elliott's approximate intertwining theorem, the algebras $A$ and $B$ are isomorphic. This completes the proof of Theorem 1.0.1, the isomorphism theorem. 


\section{Chapter 3}

\section{Existence}

In her thesis $\mathrm{L}$. $\mathrm{Li}$ [Li] improved $\mathrm{K}$. Thomsen's approximation theorem for Markov operators by removing the dependence on the operator from the hypothesis of the theorem. Recall that a Markov operator is a positive, affine, unital map between normed spaces. In our setting the maps that arise are positive, affine and contractive. We will build on Li's existence theorem to show that such maps are induced approximately (in the topology of pointwise convergence) by averages of $*$-homomorphisms between building blocks.

Theorem 3.0.1 (existence) Let $A \equiv A\left(C_{1}, D_{1}, a\right)$ be a building block algebra. For any finite set $F \subset \mathrm{AffT}^{+} A$ and $1 / 4>\varepsilon>0$, there is a natural number $N$ and an affine function $n \in \operatorname{Aff} T^{+} A,\|n\|_{A} \leq 1$ and $\inf (\iota(n)) \geq 1 / 2$ such that for any building block algebra $B \equiv B\left(C_{2}, D_{2}, b\right)$ and contractive positive linear map $\xi:\left(\operatorname{Aff} T^{+} A,\|\cdot\|_{A}\right) \rightarrow\left(\operatorname{Aff} T^{+} B,\|\cdot\|_{B}\right)$ if $\operatorname{dim}\left(D_{2}\right) \geq$ $\max \left(N / \inf _{\iota}(\xi(n)), N b / \iota(\xi(n))(0)\right)$ then there is a *-homomorphism $\psi: A \rightarrow$ $B$ (with induced map $\psi_{*}: \mathrm{Aff} T^{+} A \rightarrow \operatorname{Aff} T^{+} B$ ) such that $\left\|\xi(f)-\psi_{*}(f)\right\|_{\xi(n)}<$ 
$74 \varepsilon$ for all $f \in F$.

Proof. In section 3.1 the desingularized space $X^{\sharp}$ associated to a building block algebra $A$ and a *- homomorphism $\mathcal{L}$ from $A$ into a fixed matrix algebra over $C\left(X^{\sharp}\right)$ are constructed. In section 3.2 the map $\xi$, given in the hypothesis of the theorem is rescaled to a Markov operator from $C(\mathbb{T})$ to itself. Li's existence theorem is then applied to the rescaled map to show that it is induced approximately by an average of maps, $\phi_{i}$ from the circle to itself. Finally, in section 3.3 each map $\phi_{i}$ is lifted into the desingularized space $X^{\sharp}$; composed with $\mathcal{L}$ they induce maps from $A$ to $B$ that average to the given map $\xi$ between the affine function spaces.

\subsection{The desingularized space}

As a disclaimer we would like to note that a "desingularized space" $X^{\sharp}$ is not constructed explicitly during the course of the proof. We have used the term by analogy with the result given by Li's theorem, namely that completely positive maps between $C(X)$ and $C(Y)$ are approximate averages of $*$-homomorphisms induced by maps $\phi_{i}: Y \rightarrow X$. In our setting we will define maps $p_{s}(n, l)(\bullet, \bullet)$ and $r_{s}(m, k)(\bullet, \bullet, \bullet)$ by equations $(3.3)$ and (3.4) respectively, where by analogy the parameter "๑" is a coordinate in the desingularized space $X^{\sharp}$. In section 3.3 we will lift the finite set of maps $\phi_{i}: Y \rightarrow X$ (in our setting $X=Y=\mathbb{T}$ ) to maps from $Y$ to $X^{\sharp}$. We will use the lifted maps together with $p_{s}(n, l)(\bullet, \bullet)$ and $r_{s}(m, k)(\bullet, \bullet, \bullet)$ to define maps from $A$ to $B$.

Let $X_{p}^{\sharp}=[0,2((a+1) p-a)]$ denote the " $p$-fold extended interval". The 
desingularized space $X^{\sharp}$ is constructed by gluing together copies of $X_{k}^{\sharp}$ for various values of $k$ (picture $X_{p}^{\sharp}$ as the "backbone" of the desingularized space with different copies of $X_{k}^{\sharp}$ for various values of $k$ linking points along the backbone - this will be explained more clearly in section 3.3). Define the map $\mathcal{L}^{\prime}: A \rightarrow M_{(a+1)^{2} p}\left(C\left(X_{p}^{\sharp}\right)\right)$ up to unitary equivalence in each fibre as follows:

$$
f \mapsto\left\{\begin{aligned}
\bigoplus_{1}^{a+k / 2+1} f(t-k) & \text { for } t \in[k, k+1], k \text { even } \\
\bigoplus_{1}^{a+1} f(0) \bigoplus_{1}^{(k-1) / 2+1} f(1-(t-k)) & \text { for } t \in[k, k+1], k \text { odd }
\end{aligned}\right.
$$

By conjugating by an appropriate (discontinuous) unitary path we can ensure that the map $\mathcal{L}^{\prime}$ is continuous. Define $\mathcal{L}: \operatorname{Aff} T^{+} A \rightarrow C\left(X_{p}^{\natural}\right)$ for $f \in \operatorname{Aff} T^{+} A$ as

$$
\mathcal{L}(f)(x)=\operatorname{tr}_{(a+1)^{2} p}\left(\mathcal{L}^{\prime}\left(f^{\prime}\right)(x)\right)
$$

where $\operatorname{tr}_{(a+1)^{2} p}$ is the normalized matrix trace on $M_{(a+1)^{2} p}(\mathbb{C})$ and $f^{\prime} \in A$ is any lift of $f \in \mathrm{Aff} T^{+} A$.

Given $x_{t} \in[0,1]$, define $x_{t, l} \in X_{p}^{\sharp}$ for $1 \leq l \leq p$ as

$$
x_{t, l}=x_{t}+(l-1) 2(a+1)
$$

Then

$$
\mathcal{L}(f)\left(x_{t, l}\right)=\operatorname{tr}_{(a+1)^{2} p}\left(\bigoplus_{1}^{l(a+1)} f^{\prime}\left(x_{t}\right)\right)=\frac{l}{p} f\left(x_{t}\right)
$$

An important consequence of equation (3.1) is that for $x \in X_{l}^{\sharp} \hookrightarrow X_{p}^{\sharp}$ (we may consider a copy of $X_{l}^{\sharp}=[0,2((a+1) l-a)]$ to be canonically included in $X_{p}^{\sharp}=[0,2((a+1) p-a)]$ for $\left.l \leq p\right)$,

$$
\mid \mathcal{L}(f)(x) \leq \frac{l}{p}\|f\|_{A}
$$


Remark 3.1.1 For the remainder of this proof the term linear path joining two points $x_{t_{1}, l_{1}}, x_{t_{2}, l_{2}} \in X_{p}^{\sharp}$ will denote the path

$$
p_{s}=(1-s) x_{t_{1}, l_{1}}+s x_{t_{2}, l_{2}}
$$

for $s \in[0,1]$.

Let $l=n+m$ and $t \in[0,1]$. Up to unitary equivalence,

$$
\begin{aligned}
\mathcal{L}^{\prime}\left(f^{\prime}\right)\left(x_{t, l}\right) & =\bigoplus_{1}^{t(a+1)} f^{\prime}(t) \\
& =\bigoplus_{1}^{n(a+1)} f^{\prime}(t) \bigoplus_{1}^{m(a+1)} f^{\prime}(t) \\
& =\mathcal{L}^{\prime}\left(f^{\prime}\right)\left(x_{t, n}\right) \oplus \mathcal{L}^{\prime}\left(f^{\prime}\right)\left(x_{t, m}\right)
\end{aligned}
$$

Inspired by the above calculation, we can consider the point $x_{t, l}$ to be "split" into two components, $\left(x_{t, n}, x_{t, m}\right)$. We will not keep track of the unitary that is used to split a point since conjugating by a unitary does not change the hypothesis or conclusion of the theorem. The importance of identifying $x_{t, l}$ with $\left(x_{t, m}, x_{t, n}\right)$ is that for the purpose of this proof it is necessary to construct paths in which the first and second coordinate are varied separately. There are two general classes of paths that are considered. The first of these is constructed by joining $x_{t, n+1}$ to $x_{t, l}$ by splitting $x_{t, n+1}$ into two components and varying the second coordinate linearly inside $X_{m}^{\sharp}$ :

$$
p_{s}(n, l): x_{t, n+1} \equiv\left(x_{t, n}, x_{t, 1}\right) \rightsquigarrow\left(x_{t, n}, x_{t, m}\right) \equiv x_{t, l} .
$$

In more detail, let $\left(x_{t_{1}, n}, x_{t_{2}, m}\right) \in X_{n}^{\sharp} \times X_{m}^{\sharp}$. Define

$$
\mathcal{L}^{\prime}\left(f^{\prime}\right)\left(x_{t_{1}, n}, x_{t_{2}, m}\right)=\mathcal{L}^{\prime}\left(f^{\prime}\right)\left(x_{t_{1}, n}\right) \oplus \mathcal{L}^{\prime}\left(f^{\prime}\right)\left(x_{t_{2, m}}\right)
$$


Let $p_{s}(n, l)$, with $l=n+m$, be the path whose first component remains constant with a value of $x_{t, n}$ and whose second component joins $x_{t, 1}$ to $x_{t, m}$ linearly inside $X_{m}^{\sharp}$ (if $n=0$ then the first coordinate is null; in this case $p_{s}(n, l)$ is defined to be the linear path in $X_{m}^{\sharp}$ joining $x_{t, 1}$ to $\left.x_{t, l}\right)$. Then $\mathcal{L}(f)\left(x_{t, l}\right)=\mathcal{L}(f)\left(p_{0}(n, l)\right)$ and $\mathcal{L}(f)\left(x_{t, n+1}\right)=\mathcal{L}(f)\left(p_{1}(n, l)\right)$. This path has the useful property that the value of the function $\mathcal{L}(f)$ varies by at most $m / p\|f\|_{A}$ over the course of the path; by equation (3.2) since the nonconstant part of the path lies entirely inside $X_{m}^{\sharp}$, for $s_{1}, s_{2} \in[0,1]$ :

$$
\begin{aligned}
& \left|\mathcal{L}(f)\left(p_{s_{1}}(n, l)\right)-\mathcal{L}(f)\left(p_{s_{2}}(n, l)\right)\right| \\
& \quad=\left|\operatorname{tr}_{(a+1)^{2} p}\left(\mathcal{L}^{\prime}\left(f^{\prime}\right)\left(\left(1-s_{1}\right) x_{t, 1}+s_{1} x_{t, m}\right)-\mathcal{L}^{\prime}\left(f^{\prime}\right)\left(\left(1-s_{2}\right) x_{t, 1}+s_{2} x_{t, m}\right)\right)\right| \\
& \quad \leq m / p\|f\|_{A} .
\end{aligned}
$$

(We have used $\operatorname{tr}_{(a+1)^{2} p}$ to denote the normalized trace on $M_{(a+1)^{2} p}(\mathbb{C})$.)

The second class of general paths that are considered join $\left(x_{0, p-k}, x_{1, k}\right)$ to $\left(x_{0, p-(k+m)}, x_{1, k+m}\right)$, where $k+m \leq p$, by splitting the first coordinate into two components and varying the middle coordinate (if $k=0$ then the second coordinate is null: $\left(x_{0, p-k}, x_{1, k}\right) \equiv x_{0, p}$ for $\left.k=0\right)$. It should be noted that the non-negative integers $k$ and $m$ completely determine the path. For $s \in[0,1]$ let

$$
r_{s}(m, k)=\left(x_{0, p-(k+m)},(1-s) x_{0, m}+s x_{1, m}, x_{1, k}\right)
$$

To see that this path does begin and end at the points specified above notice that $r_{0}(m, k)=\left(x_{0, p-(k+m)}, x_{0, m}, x_{1, k}\right) \equiv\left(x_{0, p-k}, x_{1, k}\right)$ and $r_{1}(m, k)=$ $\left(x_{0, p-(k+m)}, x_{1, m}, x_{1, k}\right) \equiv\left(x_{0, p-(k+m)}, x_{1, k+m}\right)$. This path also has the property that $\mathcal{L}(f)$ varies by a small bounded amount over the course of the 
path. Let $s_{1}, s_{2} \in[0,1]$, then

$$
\begin{aligned}
& \left|\mathcal{L}(f)\left(r_{s_{1}}(m, k)\right)-\mathcal{L}(f)\left(r_{s_{2}}(m, k)\right)\right| \\
& \quad=\left|\operatorname{tr}_{(a+1)^{2} p}\left(\mathcal{L}^{\prime}\left(f^{\prime}\right)\left(\left(1-s_{1}\right) x_{0, m}+s_{1} x_{1, m}\right)-\mathcal{L}^{\prime}\left(f^{\prime}\right)\left(\left(1-s_{2}\right) x_{0, m}+s_{2} x_{1, m}\right)\right)\right| \\
& \quad \leq m / p\|f\|_{A} .
\end{aligned}
$$

There is also a relation between the values of $\mathcal{L}(f)\left(x_{0, p-k}, x_{1, k}\right)$ for $k \leq p$ and $\mathcal{L}(f)(0)$ similar to the relation given in equation (3.1):

$$
\begin{aligned}
\mathcal{L}(f)\left(x_{0, p-k,}, x_{1, k}\right) & =1 / p((p-k) f(0)+k f(1)) \\
& =1 / p((p-k) f(0)+k((a+1) / a) f(0)) \\
& =(1+k / a p) f(0) .
\end{aligned}
$$

\subsection{The rescaled map}

In Proposition 2.0.3 the isomorphism

$$
\begin{aligned}
\iota_{n}^{\prime}:\left(\operatorname{Aff} T^{+} A,\|\cdot\|_{n}\right) & \rightarrow\left(C(\mathbb{T}),\|\cdot\|_{\infty}\right) \\
f & \mapsto f / n
\end{aligned}
$$

was established for building block algebras $A$, where $n \in \operatorname{Aff} T^{+} A$ is an affine function such that $\inf n>0$ (in this section $\iota(f) \in C[0,1]_{a}$ will be identified with $f$ for all affine functions $f \in \operatorname{Aff} T^{+} A-$ for the definition of $\iota$ see Proposition 2.0.1). Under the identification given by equation (3.7), $\xi$ induces a Markov map $\xi_{\mathbb{T}}$ from $C(\mathbb{T})$ to itself that can be concretely described as follows:

$$
\xi_{\mathbb{T}}: C(\mathbb{T}) \rightarrow C(\mathbb{T})
$$




$$
\iota_{n}^{\prime}(f)=\frac{f}{n} \mapsto \frac{\xi(f)}{\xi(n)}
$$

for $f \in C[0,1]_{a}$. By Theorem 2.1 of $\mathrm{Li}[\mathrm{Li}]$ for any finite set $G \subset C(\mathbb{T})$ and $\varepsilon>0$ there exists an $N>0$ such that for any Markov map $\xi_{\mathrm{T}}$ there are $N$ homomorphisms $\phi_{i}: C(\mathbb{T}) \rightarrow C(\mathbb{T})$ such that $\left\|\xi_{\mathbb{T}}(g)-1 / N \sum_{i=1}^{N} \phi_{i}(g)\right\|<\varepsilon$ for all $g \in G$.

For our purposes we require maps $\phi_{i}$ that can be be lifted to $C[0,1]$; in more detail, let $\theta$ denote a fixed inclusion from $C(\mathbb{T})$ to $C[0,1]$ (given by identifying the subalgebra of functions which agree at the endpoints of the interval with $C(\mathbb{T})$ ); we require that there exist maps $\phi_{i}^{\prime}: C[0,1] \rightarrow C(\mathbb{T})$ such that $\phi_{i}=\phi_{i}^{\prime} \circ \theta$.

Proposition 3.2.1 Let $\theta$ denote a fixed inclusion from $C(\mathbb{T})$ to $C[0,1]$ induced by identifying the endpoints of the unit interval. Given a finite set $G \subset C(\mathbb{T})$ and $\varepsilon>0$ there exists an $N>0$ such that for any Markov map $\xi_{\mathbb{T}}: C(\mathbb{T}) \rightarrow C(\mathbb{T})$ there are $N$ homomorphisms $\phi_{i}: C[0,1] \rightarrow C(\mathbb{T})$ such that $\left\|\xi_{\mathbb{T}}(g)-1 / N \sum_{i=1}^{N} \phi_{i} \circ \theta(g)\right\|_{\infty}<2 \varepsilon$ for all $g \in G$.

Proof. The proof follows from a slight variation of an argument given by Nielsen and Thomsen in Lemma 4.1 of [NT]. Let $\left\{t_{1}, t_{2}, \ldots, t_{k}\right\}$ be a partition of $[0,1]$, and $\left\{h_{i}\right\}_{i=1}^{k}$ be a partition of unity of $C(\mathbb{T})$ such that

$$
\left\|g-\sum_{i=1}^{k} \theta(g)\left(t_{i}\right) h_{i}\right\|_{\infty}<\varepsilon
$$

for all $g \in G$. Define maps $t: C[0,1] \rightarrow \mathbb{R}^{k}$ and $s: \mathbb{R}^{k} \rightarrow C(\mathbb{T})$ as follows:

$$
t(f) \mapsto\left(f\left(t_{1}\right), f\left(t_{2}\right), \ldots, f\left(t_{k}\right)\right) \text { for } f \in C[0,1]
$$




$$
s\left(x_{1}, x_{2}, \ldots, x_{k}\right) \mapsto \sum_{i=1}^{k} x_{i} h_{i} \text { for } x_{i} \in \mathbb{R} .
$$

Let

$$
\xi_{\mathbb{T}}^{\prime}=\xi_{\mathbb{T}} \circ s \circ t
$$

Now we apply Li's theorem to the set $\theta(G) \subset C[0,1]$. By Theorem 2.1 of [Li] given a finite subset $\theta(G) \subset C[0,1]$ and $\varepsilon>0$ there exists an $N>0$ such that for any Markov map $\xi_{\mathrm{T}}^{\prime}$ there are $N$ homomorphism $\phi_{i}: C[0,1] \rightarrow C(\mathbb{T})$ such that

$$
\left\|\xi_{\mathbb{T}}^{\prime}(g)-1 / N \sum_{i=1}^{N} \phi_{i}(g)\right\|_{\infty}<\varepsilon
$$

for all $g \in \theta(G)$. The partition of unity was chosen fine enough so that

$$
\|g-s \circ t \circ \theta(g)\|_{\infty}<\varepsilon /\left\|\xi_{\mathbb{T}}\right\|_{\infty}
$$

for all $g \in G$. Applying the map $\xi_{\mathbb{\pi}}$,

$$
\left\|\xi_{\mathbb{T}}(g)-\xi_{\mathbb{T}}^{\prime}(\theta(g))\right\|_{\infty}<\varepsilon
$$

for $g \in G$. It follows from the inequalities (3.10) and (3.11) that

$$
\left\|\xi_{\mathbb{T}}(g)-1 / N \sum_{i=1}^{N} \phi_{i} \circ \theta(g)\right\|_{\infty}<2 \varepsilon
$$

for $g \in G$. This completes the proof of the proposition.

For the remainder of this section we will identify the functions in $C(\mathbb{T})$ with their image in $C[0,1]$ under the map $\theta$. Applying Proposition 3.2.1 to the finite set $\iota_{n}^{\prime}(F) \subset C([0,1])$, we can conclude that there are $N^{\prime}$ *homomorphisms $\phi_{i}: C[0,1] \rightarrow C(\mathbb{T})$ such that

$$
\left\|\xi \mathbb{T}\left(\iota_{n}^{\prime}(f)\right)-1 / N^{\prime} \sum_{i=1}^{N^{\prime}} \phi_{i}\left(\iota_{n}^{\prime}(f)\right)\right\|_{\infty}<\varepsilon .
$$


Let $\phi_{i}^{*}$ denote the map from circle to the unit interval induced by $\phi_{i}$ - By the definition of $\xi_{\mathbb{T}}$ given in equation (3.8), the above inequality becomes

$$
\left\|\frac{\xi(f)}{\xi(n)}-\frac{1}{N^{\prime}} \sum_{i=1}^{N^{\prime}} \frac{f}{n} \circ \phi_{i}^{*}\right\|_{\infty}<\varepsilon .
$$

For $y \in[0,1]$ this is equivalent to

$$
\frac{1}{\xi(n)(y)}\left|\xi(f)(y)-\frac{1}{N^{\prime}} \sum_{i=1}^{N^{\prime}} \frac{\xi(n)}{n\left(\phi_{i}^{*}\right)}(y) f\left(\phi_{i}^{*}(y)\right)\right|<\varepsilon
$$

or

$$
\left\|\xi(f)(y)-\frac{1}{N^{\prime}} \sum_{i=1}^{N^{\prime}} \frac{\xi(n)}{n\left(\phi_{i}^{*}\right)}(y) f\left(\phi_{i}^{*}(y)\right)\right\|_{\xi(n)}<\varepsilon .
$$

In subsection 3.3 it will be shown that given a map $\phi_{i}^{*}: \mathbb{T} \rightarrow[0,1]$ there exists a map $\phi_{i}^{\sharp}=[0,1] \rightarrow X^{\sharp}$ such that

$$
\frac{1}{\xi(n)(y)}\left|\frac{\xi(n)}{n\left(\phi_{i}^{*}\right)}(y) f\left(\phi_{i}^{*}(y)\right)-\mathcal{L}(f)\left(\phi_{i}^{\sharp}\right)(y)\right|<72 \varepsilon
$$

for all $y \in \mathbb{T}$.

In the remainder of this subsection we will define the affine function $n$ given in the hypothesis of the theorem and the value of the integer $p$ that is chosen for the backbone $X_{p}^{\sharp}$ of the desingularized space. Find $\delta>0$ such that if $|x|<\delta$ then

$$
|f(x)-f(0)|<\varepsilon
$$

for all $f \in F$. Let $N=(a+1)^{3} N^{\prime} / \varepsilon$. Define $n \in \mathrm{Aff} T^{+} A$ as follows:

$$
n(x)=\left\{\begin{array}{rll}
\frac{a}{a+1}+\left(1-\frac{a}{a+1}\right) \frac{x}{\delta} & \text { for } & x \leq \delta \\
1 & \text { for } & x>\delta
\end{array}\right.
$$


Let $p$ be the largest integer greater than $(a+1) \max (1 /(\operatorname{zinf} \xi(n)), b /(\varepsilon \xi(n)(0)), 4)$ such that $p(a+1)^{2} N^{r}$ is less than or equal to $\operatorname{dim}\left(D_{2}\right)$. Then

$$
\frac{a+1}{\operatorname{pinf} \xi(n)}<\varepsilon, \frac{(a+1) b}{p \xi(n)(0)}<\varepsilon
$$

and

$$
\begin{aligned}
\frac{\operatorname{dim}\left(D_{2}\right)-p(a+1)^{2} N^{\prime}}{\operatorname{dim}\left(D_{2}\right)} & <\frac{(a+1)^{2} N^{\prime}}{\operatorname{dim}\left(D_{2}\right)} \\
& =\frac{\varepsilon N}{(a+1) \operatorname{dim}\left(D_{2}\right)} \\
& <\frac{\varepsilon}{a+1} \min \left(\inf \xi(n), \frac{\xi(n)(0)}{b}\right)
\end{aligned}
$$

Such an integer $p$ always exists since

$$
\max \left(\frac{a+1}{\varepsilon \inf \xi(n)}, \frac{(a+1) b}{\varepsilon \xi(n)(0)}\right)(a+1)^{2} N^{\prime}=\max \left(\frac{N}{\inf \xi(n)}, \frac{N b}{\xi(n)(0)}\right) \leq \operatorname{dim}\left(D_{2}\right) .
$$

\subsection{Lifting to $M_{(a+1)^{2} p}\left(C\left(X^{\sharp}\right)\right)$}

In this section it will be shown that given a map $\phi:[0,1] \rightarrow[0,1]$ such that $\phi(0)=\phi(1)$ there exists a map $\phi^{\sharp}:[0,1] \rightarrow X^{\sharp}$ such that

$$
\frac{1}{\xi(n)(y)}\left|\frac{\xi(n)}{n(\phi)}(y) f(\phi(y))-\mathcal{L}(f)\left(\phi^{\sharp}(y)\right)\right|<72 \varepsilon
$$

for all $f \in F$, and such that $\mathcal{L}^{\prime}\left(f^{\prime}\right)\left(\phi^{\sharp}(y)\right)$ defines a map from $A$ to $B$ of small fibre dimension; $\operatorname{dim}\left(\mathcal{L}^{\prime}\left(f^{\prime}\right)\left(\phi^{\sharp}(y)\right)\right) \leq p(a+1)^{2}$. In order to see that $\mathcal{L}^{\prime}\left(f^{\prime}\right)\left(\phi^{\sharp}(y)\right)$ does indeed define a map from $A$ to $B$ it is enough to check that the right multiplicity of $\mathcal{L}^{\prime}\left(f^{\prime}\right)\left(\phi^{\sharp}(y)\right)$ is divisible by $b+1$, the left multiplicity is $b /(b+1)$ times the right multiplicity and that $\operatorname{dim}\left(\mathcal{L}^{\prime}\left(f^{\prime}\right)\left(\phi^{\sharp}(y)\right)\right) \leq$ 
$\operatorname{dim}\left(D_{2}\right)$ for all $f^{\prime} \in A$. The last of the three conditions follows immediately from the definition of $p$ given at the end of Section 3.2. The first two conditions can be expressed in more detail as follows; we require that:

$$
\begin{aligned}
& \mathcal{L}^{\prime}\left(f^{\prime}\right)\left(\phi^{\sharp}(0)\right)=\bigoplus_{1}^{l} \gamma(f) \\
& \mathcal{L}^{\prime}\left(f^{\prime}\right)\left(\phi^{\sharp}(1)\right)=\bigoplus_{1}^{r} \gamma(f)
\end{aligned}
$$

for all $f^{\prime} \in A$, where $r$ is divisible by $b+1, l=b /(b+1) r$ and $\gamma: A \rightarrow M_{q}(\mathbb{C})$ is a representation of $A$ such that $r q \leq \operatorname{dim}\left(D_{2}\right)$.

In this section, as in section 3.2, we will identify affine functions $f \in$ Aff $T^{+} A$ and $g \in \mathrm{Aff} T^{+} B$ with their images $\iota(f)$ and $\iota(g)$ in $C[0,1]_{a}$ and $C[0,1]_{b}$ respectively. In particular, it should be noted that $\xi(f) \in C[0,1]_{b}$ and $n(\phi) \in C(\mathbb{T}) \hookrightarrow_{\theta} C[0,1]$ (where the map $\theta$ was defined in section 3.2).

By choosing an appropriate value of $\varepsilon$ in the hypothesis of the theorem we can reduce to the case that $\|f\|_{A} \leq 1$ for all $f \in F$. Find a $\delta_{p}>0$ such that if $\left|t_{1}-t_{2}\right|<\delta_{p}$ then

$$
\left|\frac{\xi(n)}{n(\phi)}\left(t_{1}\right)-\frac{\xi(n)}{n(\phi)}\left(t_{2}\right)\right|<\frac{1}{p} .
$$

Let $\left\{y_{1}, \ldots, y_{R}\right\}$ be a $\delta_{p}$-dense partition of $[0,1]$ such that $|\phi(y)-\phi(0)|<\delta$ for $y \in\left[y_{1}, y_{2}\right]$ and if $\frac{\xi(n)}{n(\phi)}\left(y_{1}\right)<1+1 / p$ then $\frac{\xi(n)}{n(\phi)}\left(y_{2}\right)<1+1 / p$. As $\xi(n)(y) \leq 1$ and $n(\phi(y)) \geq a /(a+1)$ it follows that

$$
\left|\frac{\xi(n)}{n(\phi)}(y)\right| \leq \frac{a+1}{a} .
$$

Let us construct a continuous path $\phi^{\sharp}$ from $Y=[0,1]$ to the desingularized space $X^{\sharp}$ by constructing a sequence of paths on the intervals $\left[y_{j}, y_{j+1}\right]$ that 
agree at the endpoints. There are three different classes of paths that will be constructed depending on the values of $\frac{\xi(n)}{n(\phi)}\left(y_{j}\right)$ and $\frac{\xi(n)}{n(\phi)}\left(y_{j+1}\right)$.

Case 1: $\frac{\xi(n)}{n(\phi)}\left(y_{j}\right), \frac{\xi(n)}{n(\phi)}\left(y_{j+1}\right)<1+1 / p$.

Given an integer $\beta_{i}>1$ we can find a positive integer $l_{i} \leq p$ such that

$$
\left|\frac{\xi(n)}{n(\phi)}\left(y_{i}\right)-l_{i} / p\right|<\beta_{i} / p
$$

for $i=j, j+1$. Assume that $l_{j+1} \geq l_{j}$ (if $l_{j+1}<l_{j}$ then define the path as below, but in the opposite direction). The path $\phi^{\sharp}$ on the interval $\left[y_{j}, y_{j+1}\right]$ is defined to begin at $x_{\phi\left(y_{j}\right), l_{j}}$ and end at $x_{\phi\left(y_{j+1}\right), l_{j+1}}$. It is constructed by splitting the left endpoint into two components,

$$
x_{\phi\left(y_{j}\right), l_{j}}=\left(x_{\phi\left(y_{j}\right), l_{j}-1}, x_{\phi\left(y_{j}\right), 1}\right)
$$

and then varying each component separately. The first component follows the path given by $\phi$ for $y \in\left[y_{j}, y_{j+1}\right]$ :

$$
x_{\phi(y), l_{j}-1}
$$

The second component follows the linear path $p_{s}\left(1, l_{j+1}-l_{j}\right)$ (described by equation (3.3)) inside $X_{l_{j+1}-l_{j}}^{\sharp}$ from $x_{\phi\left(y_{j}\right), 1}$ to $x_{\phi\left(y_{j}\right), l_{j+1}-l_{j}}$ and then a linear path from $x_{\phi\left(y_{j}\right), l_{j+1}-l_{j}}$ to $x_{\phi\left(y_{j+1}\right), l_{j+1}-l_{j}}$. Let $q_{y}$ denote the path followed by the second component. For $y \in\left[y_{j}, y_{j+1}\right]$ define

$$
\phi^{\sharp}(y)=\left(x_{\phi(y), l_{j}-1}, q_{y}\right)
$$

Let us compare $\mathcal{L}(f)\left(\phi^{\sharp}(y)\right)$ and $\frac{\xi(n)}{n(\phi)}(y) f(\phi(y))$. For $y \in\left[y_{j}, y_{j+1}\right]$ :

$$
\left|\frac{\xi(n)}{n(\phi)}(y) f(\phi(y))-\mathcal{L}(f)\left(\phi^{\sharp}(y)\right)\right|
$$




$$
\leq\left|\frac{\xi(n)}{n(\phi)}(y) f(\phi(y))-\frac{l_{j}}{p} f(\phi(y))\right|+\left|\frac{l_{j}}{p} f(\phi(y))-\mathcal{L}(f)\left(\phi^{\sharp}(y)\right)\right| .
$$

Since

$$
\begin{aligned}
\left|\frac{\xi(n)}{n(\phi)}(y) f(\phi(y))-\frac{l_{j}}{p} f(\phi(y))\right| & \leq\left|\frac{\xi(n)}{n(\phi)}(y)-\frac{l_{j}}{p}\right|\|f\|_{A} \\
& \leq \frac{\beta_{j}+1}{p}
\end{aligned}
$$

it suffices to find an estimate for the second of the two terms in equation (3.19) considered above. As $q_{y} \in X_{l_{j+1}-l_{j}}^{\sharp}$, by the bound given in equation (3.2)

$$
\begin{aligned}
\left|\mathcal{L}(f)\left(q_{y}\right)\right| & \leq \frac{l_{j+1}-l_{j}}{p}\|f\|_{A} \\
& \leq \frac{\beta_{j}+\beta_{j+1}+1}{p}
\end{aligned}
$$

The second term of equation (3.19) may now be estimated as follows:

$$
\begin{aligned}
\left|\frac{l_{j}}{p} f(\phi(y))-\mathcal{L}(f)\left(\phi^{\sharp}(y)\right)\right| & =\left|\frac{l_{j}}{p} f(\phi(y))-\mathcal{L}(f)\left(x_{\phi(y), l_{j}-1}, q_{y}\right)\right| \\
& \leq\left|\frac{l_{j}}{p} f(\phi(y))-\frac{l_{j}-1}{p} f(\phi(y))\right|+\left|\mathcal{L}(f)\left(q_{y}\right)\right| \\
& \leq \frac{\beta_{j}+\beta_{j+1}+2}{p}
\end{aligned}
$$

Therefore on $\left[y_{j}, y_{j+1}\right]$,

$$
\frac{1}{\xi(n)(y)}\left|\frac{\xi(n)}{n(\phi)}(y) f(\phi(y))-\mathcal{L}(f)\left(\phi^{\sharp}(y)\right)\right| \leq \frac{2 \beta_{j}+\beta_{j+1}+3}{\xi(n)(y) p} .
$$

Case 2: $\frac{\xi(n)}{n(\phi)}\left(y_{j}\right) \frac{\xi(n)}{n(\phi)}\left(y_{j+1}\right) \geq 1+1 / p$. Assume that $\frac{\xi(n)}{n(\phi)}\left(y_{j+1}\right) \geq \frac{\xi(n)}{n(\phi)}\left(y_{j}\right)$. As $\frac{\xi(n)}{n(\phi)}(y) \leq \frac{a+1}{a}$, given an integer $\beta_{i}>0$ we can find a positive integer $k_{i} \leq p$ 
such that

$$
\left|\frac{a p+k_{i}}{a p}-\frac{\xi(n)}{n(\phi)}\left(y_{i}\right)\right|<\frac{\beta_{i}}{p}
$$

for $i=j, j+1$.

The path is constructed on the interval $\left[y_{j}, y_{j+1}\right]$ as follows: $\phi^{\sharp}$ joins $\left(x_{0, p-k_{j}}, x_{1, k_{j}}\right)$ to $\left(x_{0, p-k_{j+1}}, x_{1, k_{j+1}}\right)$ and is given by $r_{s}\left(k_{j+1}-k_{j}, k_{j}\right)$ in equation (3.4) with the parameter $s$ rescaled so that the path begins at $s=y_{j}$ and ends at $s=y_{j+1}$, As $\left|k_{j+1}-k_{j}\right| \leq a\left(\beta_{j}+\beta_{j+1}+1\right)$, by equation (3.5) for $s_{1}, s_{2} \in\left[y_{j}, y_{j+1}\right]$,

$$
\left|\mathcal{L}(f)\left(r_{s_{1}}\right)-\mathcal{L}(f)\left(r_{s_{2}}\right)\right| \leq \frac{a\left(\beta_{j}+\beta_{j+1}+1\right)}{p} .
$$

As $\frac{\xi(n)}{n(\phi)}(y)$ is within $1 / p$ of $\frac{\xi(n)}{n(\phi)}\left(y_{j}\right)$ on $\left(y_{j}, y_{j+1}\right)$ and since $1+1 / p$ is a lower bound for $\frac{\xi(n)}{n(\phi)}\left(y_{j}\right)$ it follows that

$$
\frac{\xi(n)}{n(\phi)}(y)>1
$$

for $y \in\left[y_{j}, y_{j+1}\right]$. (This is the crucial hypothesis needed for the proof of case 2 of the theorem; the argument from this point onwards will be used in case 3 as well.)

Proposition 3.3.1 If $\frac{\xi(n)}{n(\phi)}(y)>1$ then $\xi(n)(y)>a /(a+1)$ and $\phi(y) \in[0, \delta)$.

Proof. Recall that $n(x)=1$ for $x \geq \delta$ and $n(x) \geq a /(a+1)$ for $x \in[0, \delta]$. As $\xi$ is contractive, $\xi(n)(y) \leq 1$ for all $y \in[0,1]$. The hypothesis of the proposition then yields the following two inequalities:

$$
\xi(n)(y)>n(\phi(y)) \geq \frac{a}{a+1}
$$


and

$$
1 \geq \xi(n)(y)>n(\phi(y))
$$

The result follows immediately.

The conclusions of Proposition 3.3.1 together with equation (3.13), give an estimate for $f(\phi(y))$ as follows:

$$
\begin{aligned}
\frac{1}{\xi(n)(y)}|f(\phi(y))-f(0)| & <\frac{a+1}{a}|f(\phi(y))-f(0)| \\
& <\varepsilon .
\end{aligned}
$$

Let us compare $\mathcal{L}(f)\left(\phi^{\sharp}(y)\right)$ and $\frac{\xi(n)}{n(\phi)}(y) f(\phi(y))$ :

$$
\begin{aligned}
& \frac{1}{\xi(n)(y)}\left|\frac{\xi(n)}{n(\phi)}(y) f(\phi(y))-\mathcal{L}(f)\left(\phi^{\sharp}(y)\right)\right| \\
& \leq \frac{1}{\xi(n)(y)}\left(\left|\frac{\xi(n)}{n(\phi)}(y)\right||f(\phi(y))-f(0)|+\left|\frac{\xi(n)}{n(\phi)}(y) f(0)-\frac{a p+k_{j}}{a p} f(0)\right|\right. \\
& \left.\quad+\left|\frac{a p+k_{j}}{a p} f(0)-\mathcal{L}(f)\left(\phi^{\sharp}(y)\right)\right|\right) .
\end{aligned}
$$

The first of the three terms above was estimated, essentially, in equation (3.22) (recall that $\left|\frac{\xi(n)}{n(\phi)}(y)\right| \leq \frac{a+1}{a}$ by equation (3.17)); in particular

$$
\frac{1}{\xi(n)(y)}\left|\frac{\xi(n)}{n(\phi)}(y)\right||f(\phi(y))-f(0)| \leq \frac{a+1}{a} \varepsilon \leq 2 \varepsilon \text {. }
$$

The middle term can be estimated as follows:

$$
\begin{aligned}
\frac{1}{\xi(n)(y)}\left|\frac{\xi(n)}{n(\phi)}(y) f(0)-\frac{a p+k_{j}}{a p} f(0)\right| & \leq \frac{1}{\xi(n)(y)}\left|\frac{\xi(n)}{n(\phi)}(y)-\frac{a p+k_{j}}{a p}\right|\|f\|_{A} \\
& \leq \frac{\beta_{j}+1}{p \xi(n)(y)}
\end{aligned}
$$

To estimate the third term we use the bound on the variation of $\mathcal{L}(f)\left(r_{s}\left(k_{j+1}-\right.\right.$ $\left.k_{j}, k_{j}\right)$ ) given by equation (3.21) and the identity given in equation (3.6):

$$
\frac{1}{\xi(n)(y)}\left|\frac{a p+k_{j}}{a p} f(0)-\mathcal{L}(f)\left(\phi^{\sharp}(y)\right)\right|
$$




$$
\begin{aligned}
& =\frac{1}{\xi(n)(y)}\left|\mathcal{L}(f)\left(\phi^{\sharp}\left(y_{j}\right)\right)-\mathcal{L}(f)\left(\phi^{\sharp}(y)\right)\right| \\
& \leq \frac{1}{\xi(n)(y)}\left(\frac{a\left(\beta_{j}+\beta_{j+1}+1\right)}{p}\|f\|_{A}\right) \\
& \leq \frac{a\left(\beta_{j}+\beta_{j+1}+1\right)}{\xi(n)(y) p}
\end{aligned}
$$

Therefore

$$
\frac{1}{\xi(n)(y)}\left|\frac{\xi(n)}{n(\phi)}(y) f(\phi(y))-\mathcal{L}(f)\left(\phi^{\sharp}(y)\right)\right|<2 \varepsilon+\frac{a\left(2 \beta_{j}+\beta_{j+1}+2\right)}{\xi(n)(y) p}
$$

Case 3: One of $\frac{\xi(n)}{n(\phi)}\left(y_{j}\right)$ and $\frac{\xi(n)}{n(\phi)}\left(y_{j+1}\right)$ is greater than or equal to $1+1 / p$, and the other is less than $1+1 / p$.

We can assume without loss of generality that $\frac{\xi(n)}{n(\phi)}\left(y_{j+1}\right)>\frac{\xi(n)}{n(\phi)}\left(y_{j}\right)$. As $\frac{\xi(n)}{n(\phi)}(y)$ is within $1 / p$ of $\frac{\xi(n)}{n(\phi)}\left(y_{j+1}\right)$ it follows that

$$
\frac{\xi(n)}{n(\phi)}(y)>1
$$

By Proposition 3.3.1, $\xi(n)(y) \geq a /(a+1)$ and $\phi(y) \in[0, \delta)$ for $y \in\left[y_{j}, y_{j+1}\right]$. As in equations (3.18) and (3.20), given integers $\beta_{j}, \beta_{j+1}>0$ choose integers $l_{j}$ and $k_{j}$ such that

$$
\left|\frac{\xi(n)}{n(\phi)}\left(y_{j}\right)-l_{j} / p\right|<\beta_{j} / p
$$

and

$$
\left|\frac{a p+k_{j+1}}{a p}-\frac{\xi(n)}{n(\phi)}\left(y_{j+1}\right)\right|<\frac{\beta_{j+1}}{p} .
$$

Choose $\delta_{1}>0$ such that $y_{j}<\delta_{1}<y_{j+1}$. On the interval $\left[\delta_{1}, y_{j+1}\right]$ define $\phi^{\sharp}$ to be the path $r_{s}\left(k_{j+1}, 0\right)$ (given by equation (3.4), rescaled to begin at $s=\delta_{1}$ 
and end at $\left.s=y_{j+1}\right)$ that joins $\left(x_{0, p}\right)$ to $\left(x_{0, p-k_{j+1}}, x_{1, k_{j+1}}\right)$. The argument given in Case 2 then proves that

$$
\frac{1}{\xi(n)(y)}\left|\frac{\xi(n)}{n(\phi)}(y) f(\phi(y))-f\left(\phi^{\sharp}(y)\right)\right|<2 \varepsilon+\frac{a\left(2 \beta_{j}+\beta_{j}+2\right)}{\xi(n)(y) p}
$$

on this interval.

There are two steps to defining $\phi^{\sharp}$ on the interval $\left[y_{j}, \delta_{1}\right]$. The first step is to join $x_{0, l_{j}}$ to $x_{0, p}$. The second step is to join $x_{\phi\left(y_{j}\right), l_{j}}$ to $x_{0, l_{j}}$.

Choose $\delta_{2}>0$ such that $y_{j}<\delta_{2}<\delta_{1}$. Define $\phi^{\sharp}(y)$ on $\left[\delta_{2}, \delta_{1}\right]$ to be the path $p_{s}\left(l_{j}, p\right)$ defined as in equation (3.3), joining $x_{0, l_{j}}$ to $x_{0, p}$, and rescaled to begin at $s=\delta_{2}$ and end at $s=\delta_{1}$. For $y \in\left[\delta_{2}, \delta_{1}\right]$,

$$
\begin{aligned}
& \left|\frac{\xi(n)}{n(\phi)}(y) f(\phi(y))-\mathcal{L}(f)\left(\phi^{\sharp}(y)\right)\right| \\
& \quad<\quad\left|\frac{\xi(n)}{n(\phi)}(y) \| f(\phi(y))-f(0)\right|+\left|\frac{\xi(n)}{n(\phi)}(y) f(0)-\frac{l_{j}}{p} f(0)\right| \\
& \quad+\left|\frac{l_{j}}{p} f(0)-\mathcal{L}(f)\left(\phi^{\sharp}(y)\right)\right| \\
& \quad<\quad \frac{a+1}{a} \varepsilon+\frac{\beta_{j}+1}{p}\|f\|_{A}+\frac{p-l_{j}}{p}\|f\|_{A} \\
& \quad<2 \varepsilon+\frac{3 \beta_{j}+1}{p} .
\end{aligned}
$$

On $\left[y_{j}, \delta_{1}\right]$ define $\phi^{\sharp}(y)$ to be the linear path joining $x_{\phi\left(y_{j}\right), l_{j}}$ to $x_{0, l_{j}}$ inside $X_{p}^{\sharp}$. Then on this interval the same comparison made above holds, but with the sum of the three terms in equation (3.25) bounded by

$$
\frac{a+1}{a} \varepsilon+\frac{\beta_{j}+1}{p}\|f\|_{A}+\frac{l_{j}}{p} \varepsilon<3 \varepsilon+\frac{\beta_{j}+1}{p} .
$$

$$
\text { As } \begin{aligned}
\frac{\xi(n)}{n(\phi)}(y)>1, \text { and } n(x) \geq a /(a+1), \\
\frac{1}{\xi(n)(y)}<\frac{a+1}{a} .
\end{aligned}
$$


Therefore on the interval $\left[y_{j}, y_{j+1}\right]$,

$$
\frac{1}{\xi(n)(y)}\left|\frac{\xi(n)}{n(\phi)}(y) f(\phi(y))-\mathcal{L}(f)\left(\phi^{\sharp}(y)\right)\right|<6 \varepsilon+\frac{6 \beta_{j}+2}{p} .
$$

This completes the arguments for case 3 .

The map $\phi^{\sharp}(y)$ is defined on $[0,1]$ by considering one of the three cases for each of the intervals $\left[y_{j}, y_{j+1}\right]$ and making an appropriate choice of $\beta_{j}$. For intervals $\left[y_{j}, y+j+1\right], j \neq 1, R-1$ define $\phi^{\sharp}(y)$ as in case 1,2 or 3 (depending on the values of $\frac{\xi(n)}{n(\phi)}\left(y_{j}\right)$ and $\left.\frac{\xi(n)}{n(\phi)}\left(y_{j+1}\right)\right)$ with $\beta_{j}=2$. Then, using equation (3.14),

$$
\begin{aligned}
& \quad \frac{1}{\xi(n)(y)}\left|\frac{\xi(n)}{n(\phi)}(y) f(\phi(y))-\mathcal{L}(f)\left(\phi^{\sharp}(y)\right)\right| \\
& \quad<\max \left(\frac{9}{p \xi(n)(y)}, 2 \varepsilon+\frac{8 a}{p \xi(n)(y)}, 6 \varepsilon+\frac{14}{p \xi(n)(y)}\right)<20 \varepsilon
\end{aligned}
$$

on $\left[y_{2}, y_{R-1}\right]$.

For the intervals $\left[y_{1}, y_{2}\right]$ and $\left.y_{R-1}, y_{R}\right]$ there are three subcases that must be considered depending on the values of $\frac{\xi(n)}{n(\phi)}\left(y_{1}\right)$ and $\frac{\xi(n)}{n(\phi)}\left(y_{R}\right)$.

Subcase A: $\frac{\xi(n)}{n(\phi)}\left(y_{R}\right) \leq 1+1 / p$.

As $\frac{\xi(n)}{n(\phi)}\left(y_{1}\right)=\frac{b}{b+1} \frac{\xi(n)}{n(\phi)}\left(y_{R}\right)$ it follows that $\frac{\xi(n)}{n(\phi)}\left(y_{1}\right) \leq 1+1 / p$ as well. If we let $\beta_{1}=b$ then we can choose $l_{1}$ to be divisible by $b$ in equation (3.18). Let $l_{R}=\frac{b+1}{b} l_{1}$. Then

$$
\begin{aligned}
\left|\frac{l_{R}}{p}-\frac{\xi(n)}{n(\phi)}\left(y_{R}\right)\right| & =\frac{b+1}{b}\left|\frac{l_{1}}{p}-\frac{\xi(n)}{n(\phi)}\left(y_{1}\right)\right| \\
& <\frac{b+1}{p} .
\end{aligned}
$$

Therefore $\beta_{R}=b+1$ is consistent with this choice of $l_{R}$. Define the map $\phi^{\sharp}(y)$ as in case 1 or case 2 with the above values of $\beta_{1}$ and $\beta_{R}$ on the intervals 
$\left[y_{1}, y_{2},\right]$ and $\left[y_{R-1}, y_{R}\right]$. Then, on the these intervals, by equation (3.14) and the estimates given in case 1 and case 2 ,

$\frac{1}{\xi(n)(y)}\left|\frac{\xi(n)}{n(\phi)}(y) f(\phi(y))-\mathcal{L}(f)\left(\phi^{\sharp}(y)\right)\right|<\max \left(\frac{2 b+7}{p \xi(n)(y)}, 2 \varepsilon+\frac{2 b+6}{p \xi(n)(y)}\right)<10 \varepsilon$.

Finally, let us check that the endpoint multiplicities of $\mathcal{L}^{\prime}\left(f^{\prime}\right)\left(\phi^{\sharp}(y)\right.$ have been suitably chosen to define elements of $B$ for all $f^{\prime} \in A$. At the left endpoint

$$
\begin{aligned}
\mathcal{L}^{\prime}\left(f^{\prime}\right)\left(\phi^{\sharp}(0)\right) & =\mathcal{L}^{\prime}\left(f^{\prime}\right)\left(x_{\phi(0), l_{1}}\right) \\
& =\bigoplus_{1}^{l_{1}(a+1)} f^{\prime}(\phi(0))
\end{aligned}
$$

and at the right endpoint

$$
\begin{aligned}
\mathcal{L}^{\prime}\left(f^{\prime}\right)\left(\phi^{\sharp}(1)\right) & =\mathcal{L}^{\prime}\left(f^{\prime}\right)\left(x_{\phi(1), l_{R}}\right) \\
& =\bigoplus_{\substack{\iota_{R}(a+1) \\
\iota_{1}}} f^{\prime}(\phi(1)) \\
& =\bigoplus_{1}^{\frac{b+1}{b}(a+1)} f^{\prime}(\phi(0)) .
\end{aligned}
$$

Since the left endpoint multiplicity is divisible by $b$ and the right endpoint multiplicity is $(b+1) / b$ times the left endpoint multiplicity, $\mathcal{L}^{\prime}\left(\phi^{\sharp}(y)\right)$ satisfies the boundary conditions necessary to define a map from $A$ to $B$.

Subcase B: $\frac{\xi(n)}{n(\phi)}\left(y_{R}\right) \geq 1+1 / p$ and $\frac{\xi(n)}{n(\phi)}\left(y_{1}\right) \geq 1+1 / p$.

With $\beta_{1}=b$ we can find a positive integer $k_{1} \leq p$ such that $a p+k_{1}$ is divisible by $b$ in equation (3.20). Let $k_{R}=\frac{b+1}{b}\left(a p+k_{1}\right)-a p$. A similar argument to that given in equation (3.27) then proves that $\left|\frac{a p+k_{R}}{a p}-\frac{\xi(n)}{n(\phi)}\left(y_{R}\right)\right|<\frac{b+1}{p}$. It follows that $\beta_{R}=b+1$ is consistent with this choice of $k_{R}$. Define the map 
$\phi^{\sharp}$ as in case 2 or case 3 with the above values of $k_{1}$ and $k_{R}$ on the intervals $\left[y_{1}, y_{2}\right]$ and $\left[y_{R-1}, y_{R}\right]$. Therefore, on these, intervals by the estimates given in cases 1 and 2 and equation (3.14), $\frac{1}{\xi(n)(y)}\left|\frac{\xi(n)}{n(\phi)}(y) f(\phi(y))-f\left(\phi^{\sharp}(y)\right)\right|<\max \left(2 \varepsilon+\frac{2 b+6}{p \xi(n)(y)}, 6 \varepsilon+\frac{6 b+8}{p \xi(n)(y)}\right)<20 \varepsilon$. The right endpoint multiplicity can be calculated as follows:

$$
\begin{aligned}
\mathcal{L}^{\prime}\left(f^{\prime}\right)\left(\phi^{\sharp}(1)\right) & =\mathcal{L}^{\prime}\left(f^{\prime}\right)\left(x_{0, p-k_{R}}, x_{1, k_{R}}\right) \\
& =\bigoplus_{1}^{\left(p-k_{R}\right)(a+1)} f^{\prime}(0) \bigoplus_{1}^{k_{R}(a+1)} f^{\prime}(1) \\
& =\bigoplus_{1}^{\left(p-k_{R}\right)(a+1) a} e_{\infty}\left(f^{\prime}\right) \bigoplus_{1}^{k_{R}(a+1)^{2}} e_{\infty}\left(f^{\prime}\right) \\
& =\bigoplus_{1}^{\left(a p+k_{R}\right)(a+1)} e_{\infty}\left(f^{\prime}\right)
\end{aligned}
$$

for $f^{\prime} \in A$. A similar calculation made for the left endpoint multiplicity yields

$$
\mathcal{L}^{\prime}\left(f^{\prime}\right)\left(\phi^{\sharp}(0)\right)=\bigoplus_{1}^{\left(a p+k_{1}\right)(a+1)} e_{\infty}\left(f^{\prime}\right)
$$

Therefore the left endpoint multiplicity is divisible by $b$ and the right endpoint multiplicity is $(b+1) / b$ times the left endpoint multiplicity. Therefore $\mathcal{L}^{\prime}\left(\phi^{\sharp}(y)\right)$ satisfies the necessary boundary conditions to define a map from $A$ to $B$.

Subcase C: $\frac{\xi(n)}{n(\phi)}\left(y_{1}\right)<1+1 / p$ and $\frac{\xi(n)}{n(\phi)}\left(y_{R}\right) \geq 1+1 / p$.

With $\beta_{R}=b+1$ we can find a positive integer $k_{R} \leq p$ such that $a p+k_{R}$ is divisible by $a(b+1)$ in equation (3.20). Let $l_{1}=\frac{\left(a p+k_{R}\right) b}{a(b+1)}$. Then

$$
\left|\frac{l_{1}}{p}-\frac{\xi(n)}{n(\phi)}\left(y_{1}\right)\right|=\frac{b}{b+1}\left|\frac{(b+1) l_{1}}{b p}-\frac{\xi(n)}{n(\phi)}\left(y_{R}\right)\right|
$$




$$
\begin{aligned}
& =\frac{b}{b+1}\left|\frac{a p+k_{R}}{a p}-\frac{\xi(n)}{n(\phi)}\left(y_{R}\right)\right| \\
& <\frac{b}{p}
\end{aligned}
$$

Therefore $\beta_{1}=b$ is consistent with this choice of $l_{1}$. On the interval $\left[y_{R-1}, y_{R}\right]$ define $\phi^{\sharp}$ as in case 2 or case 3 with the choice of $k_{R}$ as above. On $\left[y_{1}, y_{2}\right]$ let $\phi^{\sharp}$ be a path joining $x_{0, l_{1}}$ to $x_{\phi\left(y_{2}\right), l_{2}}$. There are two steps. Find $\delta_{1}>0$ such that $y_{1}<\delta_{1}<y_{2}$. On $\left[y_{1}, \delta_{1}\right]$ we join $x_{0, l_{1}}$ to $x_{0, l_{2}}$ as in equation (3.3) with the map $p_{s}\left(l_{1}, l_{2}\right)$, then we join $x_{0, l_{2}}$ linearly to $x_{\phi\left(y_{2}\right), l_{2}}$ on the interval $\left[\delta_{1}, y_{2}\right]$. Let us show that $\frac{1}{\xi(n)(y)}\left[\frac{\xi(n)}{n(\phi)}(y) f(\phi(y))-f\left(\phi^{\sharp}(y)\right) \mid\right.$ is small along these two paths.

First let us find a upper bound for $\frac{1}{\xi(n)(y)}$. As $\frac{\xi(n)}{n(\phi)}\left(y_{1}\right)=\frac{b}{b+1} \frac{\xi(n)}{n(\phi)}\left(y_{R}\right) \geq$ $1+\frac{1}{p}$

$$
\frac{\xi(n)}{n(\phi)}(y) \geq \frac{b}{b+1}-\frac{1}{p}>\frac{1}{2}-\frac{1}{4}
$$

on the interval $\left[y_{1}, y_{2}\right]$. As $n(\phi)(y) \geq a /(a+1)$ and $p \geq 4$, it follows that

$$
\frac{1}{\xi(n)(y)}<8
$$

As $\phi(0)=\phi(1) \in[0, \delta)$ and $|\phi(y)-\phi(0)|<\delta$ for $y \in\left[y_{1}, y_{2}\right]$ it follows that, on this interval,

$$
|\phi(y)-\phi(0)|<2 \delta
$$

Therefore, on the interval $\left[y_{1}, y_{2}\right]$,

$$
\left|\frac{\xi(n)}{n(\phi)}(y)\right||f(\phi(y))-f(0)|<2 \varepsilon
$$

and

$$
\left|\frac{\xi(n)}{n(\phi)}(y)-\frac{l_{1}}{p}\right|<\frac{b}{p}
$$


Along the path from $x_{0, l_{1}}$ to $x_{0, l_{2}}$,

$$
\left|\frac{l_{1}}{p} f(0)-\mathcal{C}(f)\left(\phi^{\sharp}(y)\right)\right|<\frac{l_{2}-l_{1}}{p}<\frac{\beta_{2}+\beta_{1}+1}{p}=\frac{3+b}{p} .
$$

Along the linear path from $x_{0, l_{2}}$ to $x_{\phi\left(y_{2}\right), l_{2}}$,

$$
\begin{aligned}
& \left|\frac{l_{1}}{p} f(0)-\mathcal{L}(f)\left(\phi^{\sharp}(y)\right)\right| \\
& \quad \leq\left|\frac{l_{1}}{p} f(0)-f\left(x_{0, l_{2}}\right)\right|+\mid f\left(x_{0, l_{2}}-\mathcal{L}(f)\left(\phi^{\sharp}(y)\right) \mid\right. \\
& \quad \leq \frac{l_{2}-l_{1}}{p}\|f\|_{A}+\frac{l_{2}}{p} 2 \varepsilon \\
& \quad \leq \frac{\beta_{2}+\beta_{1}+1}{p}+2 \varepsilon=\frac{b+3}{p}+2 \varepsilon .
\end{aligned}
$$

It follows from equation (3.25) that on the interval $\left[y_{1}, y_{2}\right]$

$$
\begin{aligned}
\left|\frac{\xi(n)}{n(\phi)}(y) f(\phi(y))-\mathcal{L}(f)\left(\phi^{\sharp}(y)\right)\right| & <2 \varepsilon+\frac{b}{p}+\max \left(\frac{3+b}{p}, \frac{3+b}{p}+2 \varepsilon\right) \\
& <9 \varepsilon .
\end{aligned}
$$

Together with the upper bound on $\frac{1}{\xi(n)(y)}$, we can conclude that

$$
\frac{1}{\xi(n)(y)}\left|\frac{\xi(n)}{n(\phi)}(y) f(\phi(y))-\mathcal{L}(f)\left(\phi^{\sharp}(y)\right)\right|<72 \varepsilon
$$

on the interval $\left[y_{1}, y_{2}\right]$.

Let us check that the endpoint multiplicities are consistent. The right endpoint multiplicity can be calculated using equation (3.28) :

$$
\begin{aligned}
\mathcal{L}^{\prime}\left(f^{\prime}\right)\left(\phi^{\sharp}(1\}\right) & =\mathcal{L}^{\prime}\left(f^{\prime}\right)\left(x_{0, p-k_{R}}, x_{1, k_{R}}\right) \\
& =\bigoplus_{1}^{\left(a p+k_{R}\right)(a+1)} e_{\infty}\left(f^{\prime}\right) .
\end{aligned}
$$

The left endpoint multiplicity can be calculated as follows:

$$
\mathcal{L}^{\prime}\left(f^{\prime}\right)\left(\phi^{\sharp}(0)\right)=\mathcal{L}^{\prime}\left(f^{\prime}\right)\left(x_{0, l_{1}}\right)
$$




$$
\begin{aligned}
& =\bigoplus_{1}^{l_{1}(a+1)} f^{\prime}(0) \\
& =\bigoplus_{1}^{\frac{\left(a p+k_{R}\right) b}{a(b+1)}(a+1)}\left(\bigoplus_{1}^{a} e_{\infty}\left(f^{\prime}\right)\right) \\
& =\bigoplus_{1}^{\frac{b}{b+1}\left(a p+k_{R}\right)(a+1)} e_{\infty}\left(f^{\prime}\right) .
\end{aligned}
$$

Therefore the left endpoint multiplicity is $b /(b+1)$ times the right endpoint multiplicity and is divisible by $b$.

To summarize our results so far in section 3.3: given a map $\phi:[0,1] \rightarrow$ $[0,1]$ such that $\phi(0)=\phi(1)$, we have defined a map $\phi^{\sharp}:[0,1] \rightarrow X^{\sharp}$ such that

$$
\frac{1}{\xi(n)(y)}\left|\frac{\xi(n)}{n(\phi)}(y) f(\phi(y))-\mathcal{L}(f)\left(\phi^{\sharp}(y)\right)\right|<72 \varepsilon
$$

for all $y \in[0,1]$. (The value of $72 \varepsilon$ is the maximum of the epsilon values found in the three subcases A, B and C for intervals containing an endpoint, and the epsilon value given in equation (3.26) for intervals in the interior of $[0,1]$.) In the remainder of this section we will define the map $\psi$ that is given in the conclusion of the theorem, and check that it has the required degree of comparability with $\xi$.

Recall that in section 3.2 , a set of $N^{\prime}$ maps $\phi_{i}^{*}: \mathbb{T} \rightarrow[0,1]$ were found such that for $y \in[0,1] \hookrightarrow \mathbb{T}$,

$$
\frac{1}{\xi(n)(y)}\left|\xi(f)(y)-\frac{1}{N^{\prime}} \sum_{i=1}^{N^{\prime}} \frac{\xi(n)}{n\left(\phi_{i}^{*}\right)}(y) f \circ \phi_{i}^{*}(y)\right|<\varepsilon
$$

(see equation (3.12)). We may consider each $\phi_{i}^{*}$ to be a map from the interval to itself such that $\phi_{i}^{*}(0)=\phi_{i}^{*}(1)$. For each map $\phi_{i}^{*}$, by the arguments given 
earlier in this subsection, there exists a map $\phi_{i}^{\sharp}=[0,1] \rightarrow X^{\sharp}$ such that

$$
\frac{1}{\xi(n)(y)}\left|\frac{\xi(n)}{n\left(\phi_{i}^{*}\right)}(y) f\left(\phi_{i}^{*}(y)\right)-\mathcal{L}(f)\left(\phi_{i}^{\sharp}(y)\right)\right|<72 \varepsilon \text {. }
$$

Let

$$
\psi\left(f^{\prime}\right)=\bigoplus_{i=1}^{N^{\prime}} \mathcal{L}^{\prime}\left(f^{\prime}\right)\left(\phi_{i}^{*}\right)
$$

for $f^{\prime} \in A$. As $\mathcal{L}^{\prime}\left(f^{\prime}(y)\right) \in M_{(a+1)^{2} p}(\mathbb{C})$ for all all $y \in[0,1], \operatorname{dim}(\psi(f)(y))=$ $N^{\prime}(a+1)^{2} p$ and is less than or equal to $\operatorname{dim}\left(D_{2}\right)$ by the definition of $p$. Furthermore, the maps $\phi_{i}^{\sharp}$ were chosen so that the multiplicity of $\mathcal{L}^{\prime}\left(f^{\prime}\right)\left(\phi_{i}^{\sharp}(0)\right)$ is $b /(b+1)$ the multiplicity $\mathcal{L}^{\prime}\left(f^{\prime}\right)\left(\phi_{i}^{\sharp}(1)\right)$. Therefore, suitably conjugated by a unitary in $D_{2} \otimes C[0,1], \psi$ defines a map from $A$ to $B$.

Let $\operatorname{tr}_{D_{2}}$ and $\operatorname{tr}_{(a+1)^{2} p}$ denote the canonical normalized matrix traces on $D_{2}$ and $M_{(a+1)^{2} p}(\mathbb{C})$ respectively. Then

$$
\begin{aligned}
\psi_{*}(f)(y) & =\operatorname{tr}_{D_{2}}\left(\psi\left(f^{\prime}\right)(y)\right) \\
& =\sum_{i=1}^{N^{\prime}} \frac{(a+1)^{2} p}{\operatorname{dim} D_{2}}\left(\operatorname{tr}_{(a+1)^{2} p} \mathcal{L}^{\prime}\left(f^{\prime}\right)\left(\phi_{i}^{\sharp}(y)\right)\right) \\
& =\frac{(a+1)^{2} p}{\operatorname{dim} D_{2}} \sum_{i=1}^{N^{\prime}} \mathcal{L}(f)\left(\phi_{i}^{\sharp}(y)\right) .
\end{aligned}
$$

Let us compare $\frac{(a+1)^{2} p}{\operatorname{dim} D_{2}}$ and $\frac{1}{N^{\prime}}$, weighted with a factor of $\frac{1}{\xi(n)(y)}$ using equation (3.15):

$$
\begin{aligned}
\frac{1}{\xi(n)(y)}\left(\frac{(a+1)^{2} p}{\operatorname{dim} D_{2}}-\frac{1}{N^{\prime}}\right) & <\frac{1}{\xi(n)(y) N^{\prime}}\left(\frac{\varepsilon}{a+1} \min \left(\inf \xi(n), \frac{\xi(n)(0)}{b}\right)\right) \\
& <\varepsilon .
\end{aligned}
$$

It follows that

$$
\frac{1}{\xi(n)(y)}\left|\psi_{*}(f)-\frac{1}{N^{\prime}} \sum_{i=1}^{N^{\prime}} \mathcal{L}(f)\left(\phi_{i}^{\sharp}(y)\right)\right|<\varepsilon
$$


for all $y \in[0,1]$ and $f \in F$. We also know that

$$
\begin{aligned}
& \frac{1}{\xi(n)(y)}\left|\frac{1}{N^{\prime}} \sum_{i=1}^{N^{\prime}} \mathcal{L}(f)\left(\phi_{i}^{\sharp}(y)\right)-\frac{1}{N^{\prime}} \sum_{i=1}^{N^{\prime}} \frac{\xi(n)}{n\left(\phi_{i}^{*}\right)}(y) f(y)\right| \\
& \quad \leq \frac{1}{N^{\prime}} \sum_{i=1}^{N^{\prime}} \frac{1}{\xi(n)(y)}\left|\mathcal{L}(f)\left(\phi_{i}^{\sharp}(y)\right)-\frac{\xi(n)}{n\left(\phi_{i}^{*}\right)}(y) f(y)\right| \\
& \quad<72 \varepsilon
\end{aligned}
$$

and

$$
\frac{1}{\xi(n)(y)}\left|\frac{1}{N^{\prime}} \sum_{i=1}^{N^{\prime}} \frac{\xi(n)}{n\left(\phi_{i}^{*}\right)}(y) f(y)-\xi(f)(y)\right|<\varepsilon
$$

for all $y \in[0,1]$ and $f \in F$. Therefore

$$
\frac{1}{\xi(n)(y)}\left|\psi_{*}(f)(y)-\xi(f)(y)\right|<74 \varepsilon
$$

for all $y \in[0,1]$ and $f \in F$, or equivalently,

$$
\left\|\psi_{*}(f)-\xi(f)\right\|_{\xi(n)}<74 \varepsilon
$$

for all $f \in F$. 


\section{Chapter 4}

\section{Uniqueness}

Definition 4.0.1 The self-adjoint element

$$
h(t)=(\underbrace{1 \oplus 1 \oplus \ldots 1}_{a_{1}} \oplus t) \otimes \operatorname{Id}_{C_{1}}
$$

will be referred to as the canonical self-adjoint element of $A_{1}$.

Theorem 4.0.1 (uniqueness) Let $A_{1} \equiv A\left(C_{1}, D_{1}, a_{1}\right)$ be a building block algebra and let $h$ be the canonical self-adjoint element of $A_{1}$. For any finite set $F \subset A_{1}$ and $\varepsilon>0$ there exists a natural number $n$ and two families of functions $\left\{\delta_{i}\right\}_{i=1}^{n},\left\{h_{i}\right\}_{i=1}^{n} \subset \mathrm{Aff} T^{+} A_{1}$ such that for any two maps $\phi, \psi: \mathrm{AffT} T^{+} A_{1} \rightarrow \mathrm{Aff} T^{+} A_{2}$ (where $A_{2} \equiv A\left(C_{2}, D_{2}, a_{2}\right)$ is also a building block algebra) if for all $\tau \in T^{+} A_{2}$ there exists an $m>0$ such that $\phi_{*}\left(\delta_{n}\right)(\tau), \psi_{*}\left(\delta_{n}\right)(\tau)>m,\left|\phi_{*}\left(h_{n}\right)(\tau)-\psi_{*}\left(h_{n}\right)(\tau)\right|<m$, and $\phi(h)$ and $\psi(h)$ have at least 3 distinct eigenvalues for all $t \in[0,1]$, then there exists a unitary $U$ in the unitization of $A_{2}$ such that

$$
\left\|\phi(f)-U \psi(f) U^{*}\right\|<1011 \varepsilon \text { for all } f \in F
$$


The uniqueness theorem is proven by a straight forward application of the following lemma and theorem.

Lemma 4.0.1 Let $A_{1} \equiv A\left(C_{1}, D_{1}, a_{1}\right)$ be a building block algebra. Let $h$ be the canonical self-adjoint element of $A_{1}$. For any $\delta>0$ there exists an $n$ and two families of test functions $\left\{\delta_{i}\right\}_{i=1}^{n-1},\left\{h_{i}\right\}_{i=1}^{n} \subset$ Aff $T^{+} A_{1}$ such that for any two maps $\phi, \psi: \operatorname{Aff} T^{+} A_{1} \rightarrow \operatorname{Aff} T^{+} A_{2}$, where $A_{2} \equiv A\left(C_{2}, D_{2}, a_{2}\right)$ is also a building block algebra, if for all $\tau \in T^{+} A_{2}$ there exists a real number $m$ such that $\phi\left(\delta_{i}\right)(\tau), \psi\left(\delta_{i}\right)(\tau)>m$ and $\left|\phi\left(h_{i}\right)(\tau)-\psi\left(h_{i}\right)(\tau)\right|<m$ for $1 \leq i \leq n$ then the eigenvalues of $\phi(h)(s)$ and $\psi(h)(s)$ can be paired within $\delta$ for all $s \in[0,1]$.

Proof. The argument is essentially the same as that given in Theorem 6 of [Ell3]. Let $n$ be any positive integer greater than $3 / \delta$. We will define the two families of test functions, $\left\{h_{i}\right\}_{i=1}^{n},\left\{\delta_{i}\right\}_{i=1}^{n-1} \subset \mathrm{Aff} T^{+} A_{1}$ as follows:

$$
\iota\left(h_{i}\right)(t)=\left\{\begin{aligned}
a_{1} /\left(a_{1}+1\right) & \text { for } 0 \leq t \leq i / n \\
a_{1} /\left(a_{1}+1\right)+n(t-i) /\left(a_{1}+1\right) & \text { for } i / n \leq t \leq(i+1) / n \\
1 & \text { for }(i+1) / n \leq t \leq 1
\end{aligned}\right.
$$

and

$$
\delta_{i}=h_{i}-h_{i+1}
$$

We will define a family of characteristic functions, $\left\{k_{i}\right\}_{i=0}^{n-1}$ as follows:

$$
\iota\left(k_{i}\right)(t)=\left\{\begin{aligned}
a_{1} /\left(a_{1}+1\right) & \text { for } 0 \leq t \leq(i+1) / n \\
1 & \text { for }(i+1) / n<t \leq 1
\end{aligned}\right.
$$


Let $\operatorname{tr}_{D_{i}}$ and $\operatorname{tr}_{C_{i}}$ denote the normalized matrix trace on $D_{i}$ and $C_{i}$ respectively for $i=1,2$. From the structure theory of representations of $A_{1}$, we know that for any homomorphism $f_{s} \phi: A_{1} \rightarrow D_{2}$ and $s \in[0,1]$ there exist positive integers $M$ and $E$, and real numbers $x_{i} \in(0,1), i=1, \ldots, M$ such that $f_{s} \phi(f)$ is unitarily equivalent to

$$
\bigoplus_{i=1}^{M} f\left(x_{i}\right) \bigoplus_{1}^{E} e_{\infty}(f) \bigoplus_{1}^{Z} 0
$$

for all $f \in A_{1}$. The number of eigenvalues of $f_{s} \phi(h)$ that are greater than or equal to $i / n$ can be expressed in terms of $x_{i}$ and $E$ as

$$
\left(a_{1}+1\right) \#\left\{x_{i}: x_{i} \geq i / n\right\}+a_{1} \#\left\{x_{i}: x_{i}<i / n\right\}+E \text {. }
$$

The following calculation demonstrates that we can compute the above expression using affine function data:

$$
\begin{aligned}
\iota\left(\phi_{*}\left(k_{i}\right)\right)(s)= & \operatorname{tr}_{D_{2}}\left(f_{s} \phi\left(k_{i}\right)\right) \\
= & \frac{\operatorname{dim}\left(D_{1}\right)}{\operatorname{dim}\left(D_{2}\right)} \sum_{j=1}^{M} \operatorname{tr}_{D_{1}}\left(k_{i}^{\prime}\left(x_{j}\right)\right)+\frac{\operatorname{dim}\left(C_{1}\right)}{\operatorname{dim}\left(D_{2}\right)} \sum_{j=1}^{E} \operatorname{tr}_{C_{1}}\left(e_{\infty}\left(k_{i}^{\prime}\right)\right) \\
= & \frac{\operatorname{dim}\left(D_{1}\right)}{\operatorname{dim}\left(D_{2}\right)} \sum_{j=1}^{M} \iota\left(k_{i}\right)\left(x_{i}\right)+\frac{\operatorname{dim}\left(C_{1}\right)}{\operatorname{dim}\left(D_{2}\right)} \sum_{j=1}^{E} \iota\left(m_{1}\right)(1) \\
= & \frac{1}{\operatorname{dim}\left(D_{2}\right)}\left(\left(a_{1}+1\right) \#\left\{x_{i}: x_{i} \geq i / n\right\}\right. \\
& \left.+a_{1} \#\left\{x_{i}: x_{i}<i / n\right\}+E\right)
\end{aligned}
$$

where $k_{i}^{\prime}$ is any lift of $k_{i}$. From the hypothesis of the lemma we know that for every $s \in[0,1]$ there exists an $m^{\prime}>0$ such that

$$
\left|\iota\left(\phi_{*}\left(h_{i}\right)\right)(s)-\iota\left(\psi_{*}\left(h_{i}\right)\right)(s)\right|<m^{\prime}
$$


and

$$
\iota\left(\phi_{*}\left(\delta_{i}\right)\right)(s), \iota\left(\psi_{*}\left(\delta_{i}\right)\right)(s)>m^{\prime}
$$

From the above equations, as $\delta_{i}=h_{i}-h_{i+1}$,

$$
\begin{aligned}
\iota\left(\phi_{*}\left(h_{i}\right)\right)(s) & =\iota\left(\phi_{*}\left(h_{i+1}\right)\right)(s)+\iota\left(\phi_{*}\left(\delta_{i}\right)\right)(s) \\
& >\iota\left(\phi_{*}\left(h_{i+1}\right)\right)(s)+m^{\prime} \\
& >\iota\left(\psi_{*}\left(h_{i+1}\right)\right)(s)
\end{aligned}
$$

and

$$
\iota\left(\psi_{\star}\left(h_{i}\right)\right)(s) \geq \iota\left(\phi_{*}\left(h_{i+1}\right)\right)(s)
$$

As $k_{i-1}>h_{i}>k_{i}$,

$$
\iota\left(\phi_{*}\left(k_{i-1}\right)\right)(s)>\iota\left(\phi_{*}\left(h_{i}\right)\right)(s) \geq \iota\left(\psi_{*}\left(h_{i+1}\right)\right)(s)>\iota\left(\phi_{*}\left(k_{i+1}\right)\right)(s)
$$

and

$$
\iota\left(\psi_{*}\left(k_{i-1}\right)\right)(s)>\iota\left(\psi_{*}\left(h_{i}\right)\right)(s) \geq \iota\left(\phi_{*}\left(h_{i+1}\right)\right)(s)>\iota\left(\psi_{*}\left(k_{i+1}\right)\right)(s)
$$

for $i=1, \ldots, n-2$. Therefore, by equation (4.2) there are at least as many eigenvalues of $\phi(h)(s)$ which are greater than or equal to $(i-1) / n$ as there are eigenvalues of $\psi(h)(s)$ which are greater than or equal to $(i+1) / n$ for $i=1, \ldots, n-2$. The same also relation holds with $\phi(h)(s)$ and $\psi(h)(s)$ interchanged. It follows that we can match the eigenvalues in descending order within $3 / n$, and hence within $\delta$.

Theorem 4.0.2 Let $A_{1} \equiv A\left(C_{1}, D_{1}, a_{1}\right)$ be building block algebra and $h$ its canonical self-adjoint element. For any finite set $F \subset A_{1}$ and $\varepsilon>0$ there 
exists a $\delta>0$ such that for any two maps $\phi, \psi: A_{1} \rightarrow A_{2}$ (where $A_{2} \equiv$ $A\left(C_{2}, D_{2}, a_{2}\right)$ is also a building block algebra), if the eigenvalues of $\phi(h)(t)$ and $\psi(h)(t)$ can be paired within $\delta$, and there are at least 3 distinct eigenvalues for all $t \in[0,1]$, then there exists a unitary $U$ in the unitization of $A_{2}$ such that

$$
\left\|\phi(f)-U \psi(f) U^{*}\right\|<1009 \varepsilon \text { for all } f \in F
$$

Proof. The argument proceeds in four steps. In Section 4.1 we construct certain standard maps $\phi^{\prime}$ and $\psi^{\prime}$ from $A_{1}$ to $C[0,1] \otimes D_{2}$ that are approximately unitarily equivalent to $\phi$ and $\psi$ respectively by unitaries in $C[0,1] \otimes D_{2}$. (A notion of standard maps between building blocks was introduced in [EGJS]. Our construction is completely different from theirs, but the application - to proving a uniqueness theorem for arbitrary maps between building blocks - is the same.) In Section 4.2 we show that $\phi^{\prime}$ and $\psi^{\prime}$ are sufficiently close on the given finite set of elements; for all $f \in F$,

$$
\left\|\phi^{\prime}(f)-\psi^{\prime}(f)\right\|<7 \varepsilon
$$

In Section 4.3 unitaries $U_{1}$ and $U_{2}$ are constructed that are sufficiently close to each other with respect to the finite set $F$, and twist the standard maps $\phi^{\prime}, \psi^{\prime}$ into $A_{2}$ :

$$
\operatorname{Ad}_{U_{1}} \phi^{\prime}, \operatorname{Ad}_{U_{2}} \psi^{\prime}: A_{1} \rightarrow A_{2}
$$

Finally, in Section 4.4 we apply Jiang and Su's argument (essentially an application of Lemma 5.1 of [JS]) to show that the given maps $\phi$ and $\psi$ are approximately unitarily equivalent to $\operatorname{Ad}_{U_{1}} \phi^{\prime}$ and $\operatorname{Ad}_{U_{2}} \psi^{\prime}$, where the unitary equivalence is given by unitaries in the unitization of $A_{2}$. 
Remark 4.0.1 By twisting by a unitary if necessary we can assume that the maps $L, R: C \rightarrow D$ given in the definition of a building block have the form

$$
\begin{aligned}
& L(c)=\bigoplus_{1}^{a} c \bigoplus_{1}^{\operatorname{dim}(c)} 0 \\
& R(c)=\bigoplus_{1}^{a+1} c
\end{aligned}
$$

where $c \in C$. Furthermore, as $A(C, D, a)=A(\mathbb{C}, D, a) \otimes C$, we can reduce to the case that $A_{1}=A\left(\mathbb{C}, D_{1}, a_{1}\right)$.

Remark 4.0.2 Since the standard maps are constructed fibrewise, the term standard map, or map in standard form, will also be used for maps from $A_{1}$ to $D_{2}$.

\subsection{Constructing the standard maps}

The standard maps are constructed fibrewise and in two steps. The first step is to associate a sequence of real numbers, and a pair of positive integers (satisfying certain compatibility conditions) to a a finite dimensional representation of a building block algebra. This is accomplished by Lemma 4.1.1. The second step, in some sense, undoes the first; given a finite sequence of real numbers and a pair of positive integers we construct a representation Std of the building block algebra $A_{1}$.

Since $F$ is a finite set of functions, there exists a $\delta^{\prime}>0$ such that if $\left|t_{0}-t_{1}\right|<\delta^{\prime}$ then $\left\|f\left(t_{0}\right)-f\left(t_{1}\right)\right\|<\varepsilon / 2$ for all $f \in F$. Let

$$
\delta=\min \left(\boldsymbol{\delta}^{\prime}, \varepsilon / \pi\right)
$$


Definition 4.1.1 Let $e_{s}$ and $f_{s}$ denote evaluation at $s, 0 \leq s \leq 1$ for the algebras $A_{1}$ and $A_{2}$ respectively. Let $e_{\infty}$ and $f_{\infty}$ denote evaluation at the irreducible fibre at infinity for $A_{1}$ and $A_{2}$ respectively. Then $e_{s}: A_{1} \rightarrow D_{1}$, $e_{\infty}: A_{1} \rightarrow C_{1}, f_{s}: A_{2} \rightarrow D_{2}$ and $f_{\infty}: A_{2} \rightarrow C_{2}$

The following equations are a consequence of the mapping torus construction given to define the building blocks:

$$
\begin{aligned}
& e_{0}=\bigoplus_{1}^{a_{1}} e_{\infty} \oplus 0 \\
& e_{1}=\bigoplus_{1}^{a_{1}+1} e_{\infty} .
\end{aligned}
$$

(Similar relations hold for $f_{0}$ and $f_{1}$, but with $a_{2}$ replacing $a_{1}$ in the above equations.) The first step to defining a standard map is the following lemma about decomposing finite dimensional representations of building block algebras.

Lemma 4.1.1 Given a finite dimensional representation $\alpha: A_{1} \rightarrow M_{m}(\mathbb{C})$, where $A_{1}$ is a building block, there is a unique sequence $1 \geq s_{1} \geq s_{2} \geq \cdots \geq$ $s_{F} \geq 0$, and unique positive integers $E$ and $Z$ such that:

(i) $E<a_{1}+1$

(ii) $E<a_{1}$ and $s_{i}<1$ for all $i=1, \ldots, F$ if $Z>0$

(iii) $\alpha$ is unitarily equivalent to $e_{s_{1}} \oplus e_{s_{2}} \oplus \cdots \oplus e_{s_{F}} \bigoplus_{1}^{E} e_{\infty} \bigoplus_{1}^{Z} 0$.

Proof. Every irreducible representation of $A_{1}$ is unitarily equivalent 
to $e_{\infty}$ or $e_{s}$, for some $s \in(0,1)$. Therefore, for any representation $\alpha$ of $A_{1}$ there exists a decreasing sequence $1>s_{1}^{\prime} \geq s_{2}^{\prime} \geq \cdots \geq s_{F^{\prime}}^{\prime}>0$, and positive integers $E^{\prime}$ and $Z^{\prime}$ such that $\alpha$ is unitarily equivalent to

$$
e_{s_{1}^{\prime}} \oplus e_{s_{2}^{\prime}} \oplus \cdots \oplus e_{s_{F^{\prime}}^{\prime}} \bigoplus_{i=1}^{E^{\prime}} e_{\infty} \bigoplus_{i=1}^{Z^{\prime}} 0
$$

We can find a decomposition for $\alpha$ that satisfies conditions (i), (ii) and (iii) of the lemma by grouping evaluations at infinity together with zeros as in equation (4.4), and then grouping suitably many leftover evaluations at infinity together as in equation (4.5). A more detailed description is as follows. Let $E^{\prime}=a_{1} q_{E^{\prime}}+r_{E^{\prime}}, 0 \leq r_{E^{\prime}}<a_{1}$. There are two cases.

Case 1: $Z^{\prime} \leq q_{E^{\prime}}$.

Let $Z=0$. Group together $Z^{\prime}$ zeros with $a_{1} Z^{\prime}$ evaluations at infinity as in (4.4) to form $Z^{\prime}$ evaluations at zero. Let $a_{1}\left(q_{E^{\prime}}-Z^{\prime}\right)+r_{E}=\left(a_{1}+1\right) q_{E^{\prime \prime}}+E$, $0 \leq E<a_{1}+1$. Group together $\left(a_{1}+1\right) q_{E^{\prime \prime}}$ evaluations at infinity as in (4.5) to form $q_{E^{\prime \prime}}$ evaluations at one. Then $\alpha$ is unitarily equivalent to

$$
\bigoplus_{1}^{Z^{\prime}} e_{0} \oplus e_{s_{1}^{\prime}} \oplus \cdots \oplus e_{s_{F^{\prime}}^{\prime}} \bigoplus_{1}^{q_{E^{\prime \prime}}} e_{1} \bigoplus_{1}^{E} e_{\infty}
$$

Case 2: $Z^{\prime}>q_{E^{r}}$.

Let $E=r_{E^{\prime}}$ and $Z=Z^{r}-q_{E^{\prime}}$. Group together $q_{E^{\prime}}$ zeros with $a_{1} q_{E^{\prime}}$ evaluations at infinity as in (4.4) to form $q_{E^{r}}$ evaluations at zero. Then $\alpha$ is unitarily equivalent to

$$
\bigoplus_{1}^{q E^{\prime}} e_{0} \oplus e_{s_{1}^{\prime}} \oplus \cdots \oplus e_{s_{F^{\prime}}^{\prime}} \bigoplus_{1}^{E} e_{\infty} \bigoplus_{1}^{Z} 0
$$


By the arguments given in Case 1 and Case 2, $0 \leq E \leq a_{1}$, and if $Z>0$ then $E<a_{1}$ and $s_{i}<1$ for all $i=1, \ldots, F$; this determines a sequence $1 \geq s_{1} \geq s_{2} \geq \cdots \geq s_{F} \geq 0$ such that $\alpha$ is unitarily equivalent to

$$
e_{s_{1}} \oplus e_{s_{2}} \oplus \cdots \oplus e_{s_{F}} \bigoplus_{i=1}^{E} e_{\infty} \bigoplus_{i=1}^{Z} 0 .
$$

To prove uniqueness, let $1 \geq s_{1}^{\prime} \geq s_{2}^{\prime} \geq \ldots s_{F^{r}}^{\prime} \geq 0$ be a sequence of real numbers, $E^{\prime}$ and $Z^{\prime}$ positive integers satisfying conditions (i), (ii) and (iii) of the lemma. The evaluation map, $e_{t}$ is irreducible except for $t=0,1$. Assuming that $t \neq 0,1$, then by condition (iii), $s_{t} \in\left(s_{i}^{\prime}\right)_{1}^{F^{\prime}}$ and $s_{t}^{\prime} \in\left(s_{i}\right)_{1}^{F}$. This proves that the sequences are identical, except possibly differing in the number of ones and zeros; we can therefore reduce to the case that $s_{i}$ and $s_{i}^{\prime}$ are equal to zero or one for all values of $i$.

Suppose that $s_{i}=0$ for $i=1, \ldots, q$ and $s_{i}=1$ for $i=q+1, \ldots, F$. Then $e_{s_{1}} \oplus \cdots \oplus e_{s_{F}} \bigoplus_{1}^{E} e_{\infty} \bigoplus_{1}^{Z} 0$ is unitarily equivalent to

$$
\bigoplus_{1}^{a_{1} q+\left(a_{1}+1\right)(F-q)+E} e_{\infty} \bigoplus_{1}^{q+Z} 0
$$

Similarly, suppose that $s_{i}^{\prime}=0$ for $i=1, \ldots, q^{\prime}$ and $s_{i}=1$ for $i=q^{\prime}+1, \ldots, F^{\prime}$. Then $e_{s_{1}^{\prime}} \oplus \cdots \oplus e_{s_{F}^{\prime}} \bigoplus_{1}^{E^{r}} e_{\infty} \bigoplus_{1}^{Z^{\prime}} 0$ is unitarily equivalent to

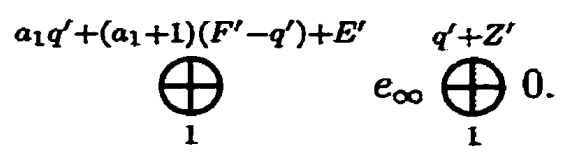

Noting that $e_{\infty}$ is irreducible, and comparing the two representations,

$$
\begin{aligned}
a_{1} q+\left(a_{1}+1\right)(F-q)+E & =a_{1} q^{\prime}+\left(a_{1}+1\right)\left(F^{\prime}-q^{\prime}+E^{\prime}\right) \\
q+Z & =q^{\prime}+Z^{\prime} .
\end{aligned}
$$

Case 1: $Z=0$ and $Z^{\prime}>0$. 
By condition (ii) of the lemma, if $Z^{\prime}>0$, then $q^{\prime}=F^{\prime}$ and $E^{\prime}<a_{1}$. Then the two equations, above, reduce to:

$$
\begin{aligned}
a_{1} q+\left(a_{1}+1\right)(F-q)+E & =a_{1} F^{\prime}+E^{\prime} \\
q & =F^{\prime}+Z^{\prime} .
\end{aligned}
$$

Isolating $F^{\prime}$ in the second equation and substituting into the first,

$$
\left(a_{1}+1\right)(F-q)+E+a_{1} Z^{\prime}=E^{\prime} .
$$

Since $Z^{\prime}>0$ and $E^{\prime}<a_{1}$ the above equation contradicts our assumption that $Z=0$ and $Z^{\prime}>0$.

Case 2: $Z=0$ and $Z^{\prime}=0$.

Then, equation (4.7) reduces to $q=q^{\prime}$. Substituting into equation (4.6),

$$
\left(a_{1}+1\right) F+E=\left(a_{1}+1\right) F^{\prime}+E^{\prime} .
$$

Since $E, E^{\prime}<a_{1}+1, F=F^{\prime}$ and $E=E^{\prime}$.

Case 3: $Z>0$ and $Z^{\prime}>0$.

Then by condition (ii) of the lemma, $q^{\prime}=F^{\prime}, q=F, E<a_{1}$ and $E^{\prime}<a_{1}$. Equation (4.6) reduces to

$$
a_{1} F^{\prime}+E^{\prime}=a_{1} F+E .
$$

It follows that $F=F^{\prime}$ and $E=E^{\prime}$. This concludes the proof of the lemma.

The remainder of this section will be devoted to constructing maps $\operatorname{Std}\left(s_{1}, s_{2}, \ldots, s_{F}, E, Z\right): A_{1} \rightarrow M_{m}(\mathbb{C})$ given a sequence of real numbers 
$1 \geq s_{1} \geq s_{2} \geq \cdots \geq s_{F} \geq 0$ and positive integers $E$ and $Z$. There are three cases.

Case 1: $Z=0$. Define the map as follows:

$$
\operatorname{Std}\left(s_{1}, s_{2}, \ldots, s_{F}, E, Z\right)=e_{s_{1}} \oplus e_{s_{2}} \oplus \cdots \oplus e_{s_{F}} \bigoplus_{1}^{E} e_{\infty}
$$

Case 2: $0 \leq Z<a_{1}-E$. The following parameterized family of unitaries will be used repeatedly in Cases 2, 3, and 4:

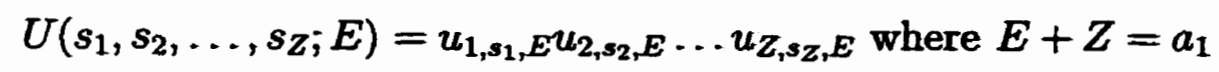

and

$$
U^{\prime}\left(s_{1}, s_{2}, \ldots, s_{Z} ; E\right)=u_{1, s_{1}, E}^{\prime} u_{2, s_{2}, E}^{\prime} \ldots u_{Z, s_{Z}, E}^{\prime} \text { where } E+Z<a_{1}
$$

The set of unitaries $u_{i, 1, E}$ for $i=1, \ldots, Z$ are defined on the Hilbert space $\mathcal{H}_{k}$ with basis $\left\{\xi_{i}\right\}_{1}^{k}$ to act as the unitary

$$
\left[\begin{array}{ll}
0 & 1 \\
1 & 0
\end{array}\right]
$$

on the subspace with basis $\left\{\xi_{m+\left(a_{1}+1\right) i}, \xi_{m+Z\left(a_{1}+1\right)+E+i}\right\}$ and as the identity on the rest of $\mathcal{H}_{k}$, where $m=k-\left(Z\left(a_{1}+1\right)+E+Z+1\right)$. In more detail,

$$
\begin{aligned}
u_{i, 1, E}\left(\xi_{m+\left(a_{1}+1\right) i}\right) & =\xi_{m+Z\left(a_{1}+1\right)+E+i} \\
u_{i, 1, E}\left(\xi_{m+Z\left(a_{1}+1\right)+E+i}\right) & =\xi_{m+\left(a_{1}+1\right) i} \\
u_{i, 1, E}\left(\xi_{j}\right) & =\xi_{j} \text { for } j \neq m+\left(a_{1}+1\right) i, m+Z\left(a_{1}+1\right)+E+i .
\end{aligned}
$$


Let $u_{i, t, E}$ be a unitary path joining $u_{i, 0, E}=$ Id to $u_{i, 1, E}$ in the subspace spanned by $\xi_{m+\left(a_{1}+1\right) i}$ and $\xi_{m+Z\left(a_{1}+1\right) E+i}$ such that

$$
\left\|u_{i, t_{1}, E}-u_{i, t_{2}, E}\right\| \leq \pi\left(t_{1}-t_{2}\right)
$$

The family of unitaries, $u_{i, t, E}^{\prime}, i=1, \ldots, Z$ and $t \in[0,1]$ are defined in a similar way. The unitary $u_{i, 1, E}^{\prime}$ is defined by the matrix given in equation (4.10) on the subspace with basis $\left\{\xi_{m^{\prime}+\left(a_{1}+1\right) i_{i}} \xi_{m^{\prime}+Z\left(a_{1}+1\right)+E+Z}\right\}$ and as the identity on the rest of $\mathcal{H}_{k}$, where $m^{\prime}=k-\left(Z\left(a_{1}+1\right)+E+Z\right)$. We can define $u_{i, t, E}^{\prime}$ to be any unitary path satisfying equation (4.11) joining the identity to $u_{i, 1, E}^{\prime}$.

Remark 4.1.1 The unitary $U\left(s_{1}, s_{2}, \ldots, s_{Z} ; E\right)$ acts as the identity except on the subspace

$$
\operatorname{sp}\left\{\xi_{m+\left(a_{1}+1\right) i}\right\}_{i=1}^{Z} \cup \operatorname{sp}\left\{\xi_{m+Z\left(a_{1}+1\right)+E+i}\right\}_{i=1}^{Z} \subset \operatorname{sp}\left\{\xi_{i}\right\}_{i=k-\left(a_{1}+1\right)^{2}}^{k}
$$

This observation will prove to be important for making estimates later in the proof of the uniqueness theorem.

Remark 4.1.2 The unitary $U\left(s_{1}, s_{2}, \ldots, s_{Z} ; E\right)$ is completely determined by the sequence of real numbers $s_{1}, s_{2}, \ldots, s_{Z}$ and the positive integer $E$ once the dimension of the Hilbert space on which it is acting is specified. For the remainder of the proof of this theorem the dimension of the Hilbert space will not be explicitly specified, but $U\left(s_{1}, s_{2}, \ldots, s_{Z} ; E\right)$ will only arise in the context of its adjoint action on a finite dimensional representation of a building block algebra; the dimension of the Hilbert space on which it acts will then be assumed to be the same as the dimension of the space on which the building block algebra is represented. The above comments also apply to 
$U^{\prime}\left(s_{1}, s_{2}, \ldots, s_{Z} ; E\right)$

Let

$$
R=\operatorname{Ad}_{U^{\prime}\left(s_{1}, s_{2}, \ldots, s_{Z} ; E\right)}\left(e_{s_{1}} \oplus e_{s_{2}} \oplus \cdots \oplus e_{s_{Z}} \bigoplus_{1}^{E} e_{\infty} \bigoplus_{1}^{Z} 0\right) .
$$

Define the map as follows:

$$
\operatorname{Std}\left(s_{1}, s_{2}, \ldots, s_{F}, E, Z\right)=e_{s_{Z+1}} \oplus e_{s_{Z+2}} \oplus \cdots \oplus e_{s_{F}} \oplus R
$$

Case 3: $Z \geq a_{1}-E$. Define two representations of $A_{1}$ as follows:

$$
R=\operatorname{Ad}_{U\left(s_{1}, s_{2}, \ldots, s_{a_{1}-E} ; E\right)}\left(e_{s_{1}} \oplus e_{s_{2}} \oplus \cdots \oplus e_{s_{a_{1}-E}} \bigoplus_{1}^{E} e_{\infty} \bigoplus_{1}^{a_{1}+1-E} 0\right)
$$

and

$$
L=e_{s_{a_{1}-E+1}} \oplus e_{s_{a_{1}-E+2}} \oplus \cdots \oplus e_{s_{F}} \oplus R .
$$

Define a unitary shift $V_{m}$ on a finite dimensional Hilbert space, $\mathcal{H}_{m}$ of dimension at least $a_{1}+1$ as follows:

$$
V_{m}\left(\xi_{i}\right)=\left\{\begin{array}{rll}
\xi_{m-\left(a_{1}+1\right)+i} & \text { for } & i=1,2, \ldots, a_{1}+1 \\
\xi_{i-\left(a_{1}+1\right)} & \text { for } & i>a_{1}+1 .
\end{array}\right.
$$

(By assumption the are at least 3 distinct eigenvalues for $\phi(h)(s)$ and in particular at least one eigenvalue that is neither 0 nor 1 . Therefore $F \geq 1$ and hence $L$ is represented on a Hilbert space of dimension at least $a_{1}+1$.) For the remainder of the proof of this theorem, the Hilbert space on which $V_{m}$ acts will not be explicitly specified (the subscript will be dropped), however in all cases $V$ will act by an adjoint action on a representation of a building block; the dimension of the space on which $V$ acts will be the same as the 
dimension of the space on which the algebra is represented. (The same is true for the unitary $U\left(s_{1}, \ldots, s_{Z} ; E\right)$ - see Remark 4.1.2.)

Given $M: A_{1} \rightarrow M_{\bullet}(\mathbb{C})$, where

$$
M=e_{t_{1}} \oplus e_{t_{2}} \oplus \cdots \oplus e_{t_{r}} \oplus M^{\prime}
$$

for some finite dimensional representation $M^{\prime}$ of $A_{1}$, define two maps $\iota_{\tau}$ : $\left(A_{1} \rightarrow M_{\bullet}(\mathbb{C})\right) \rightarrow\left(A_{1} \rightarrow M_{\bullet+a_{1}+1}(\mathbb{C})\right)$ and $\iota_{r, r^{r}}^{\prime}:\left(A_{1} \rightarrow M_{\bullet}(\mathbb{C})\right) \rightarrow\left(A_{1} \rightarrow\right.$ $\left.M_{\bullet+r}(\mathbb{C})\right)$ as follows:

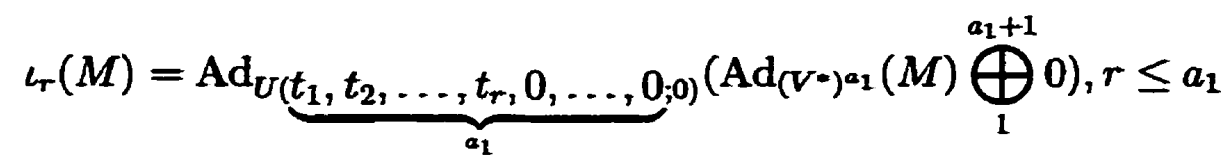

and

$$
\iota_{r, r^{\prime}}^{\prime}(M)=\operatorname{Ad}_{U^{\prime}}(\underbrace{\left.t_{1}, t_{2}, \ldots, t_{r}, 0, \ldots, 0 ; 0\right)}_{r^{\prime}}\left(\left(\operatorname{Ad}_{\left(V^{*}\right)^{r}}(M) \bigoplus_{1}^{r^{\prime}} 0\right), r \leq r^{\prime}<a_{1} .\right.
$$

By positive integer division, let

$$
Z-\left(a_{1}+1-E\right)=k_{Z}\left(a_{1}+1\right)+r_{Z}, 0 \leq r_{Z}<a_{1}+1, k_{Z} \geq 0
$$

and

$$
F-\left(a_{1}-E\right)=k\left(a_{1}\right)+r, 0 \leq r<a_{1}, k \geq 0
$$

There are three subcases for defining $\operatorname{Std}\left(s_{1}, s_{2}, \ldots, s_{F}, E, Z\right)$.

Subcase 3.1: If $k_{Z} \leq k$, and $r_{Z} \leq r$ if $k_{Z}=k$ then define the map as follows:

$$
\operatorname{Std}\left(s_{1}, s_{2}, \ldots, s_{F}, E, Z\right)=\iota_{r Z, r_{Z}}^{\prime}\left(\iota_{a_{1}}\right)^{k_{Z}}(L) .
$$

Subcase 3.2: If $k_{Z}=k$ and $r_{Z}>r$ then define the map as follows:

$$
\operatorname{Std}\left(s_{1}, s_{2}, \ldots, s_{F}, E, Z\right)=\iota_{r, r Z}^{\prime}\left(\iota_{a_{1}}\right)^{k_{Z}}(L)
$$


Subcase 3.3: If $k_{Z}>k$ then define the map as follows:

$$
\operatorname{Std}\left(s_{1}, s_{2}, \ldots, s_{F}, E, Z\right)=\iota_{0, r Z}^{\prime}\left(\iota_{0}\right)^{k_{Z}-k-1} \iota_{r}\left(\iota_{a_{1}}\right)^{k}(L)
$$

Remark 4.1.3 It's possible to give a more explicit construction for $\left(\iota_{a_{1}}\right)^{m}(L)$ for $m \leq k$ :

$$
\left(\iota_{a_{1}}\right)^{m}(L)=e_{s_{a_{1}-E+m\left(a_{1}\right)+1}} \oplus \cdots \oplus e_{s_{F}} \oplus R \bigoplus_{j=0}^{m-1} \mathcal{S}_{j}
$$

where

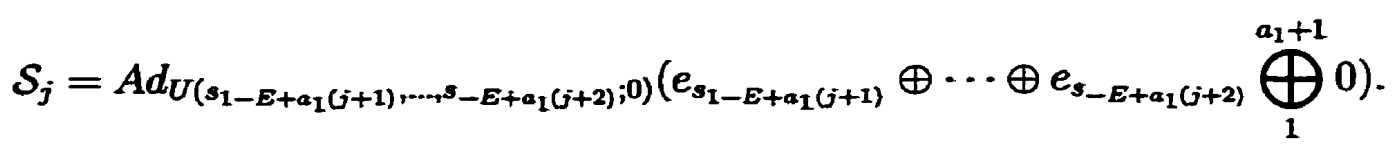

(The notation $\mathcal{S}\left(s_{1}, \ldots, s_{a_{1}}\right)=A d_{U\left(s_{1}, \ldots, s_{a_{1}}\right)}\left(e_{s_{1}} \oplus \cdots \oplus e_{s_{a_{1}}} \bigoplus_{1}^{a_{1}+1} 0\right)$ will also be used in the sequel.)

The standard maps $\phi^{\prime}, \psi^{\prime}: A_{1} \rightarrow C[0,1] \otimes D_{2}$ associated with the maps $\phi$ and $\psi$ between the building block algebras $A_{1}$ and $A_{2}$ are constructed fibrewise, in two steps. By Lemma 4.1.1, for any $s \in[0,1]$ we can find a unique sequence of real numbers $1 \geq s_{1} \geq \cdots \geq s_{F_{1}} \geq 0$ and a pair of positive integers $E_{1}, Z_{1}$ satisfying conditions (i) and (ii) of the lemma, such that $f_{s} \phi$ is unitarily equivalent to $e_{s_{1}} \oplus \cdots \oplus e_{s_{F_{1}}} \oplus_{1}^{E_{1}} e_{\infty} \oplus_{1}^{Z_{1}} 0$. Similarly we can find a positive sequence $1 \geq t_{1} \geq \cdots \geq t_{F_{2}} \geq 0$ and a pair of positive integers $E_{2}, Z_{2}$ for $f_{s} \psi$. The second step is to define the standard map at the fibre $s$ as the representation given by evaluating the map Std on the data given above:

$$
f_{s} \phi^{\prime}=\operatorname{Std}\left(s_{1}, \ldots, s_{F_{1}}, E_{1}, Z_{1}\right)
$$




$$
f_{s} \psi^{\prime}=\operatorname{Std}\left(t_{1}, \ldots, t_{F_{2}}, E_{2}, Z_{2}\right)
$$

This completes the construction of the standard maps associated with the homomorphisms $\phi$ and $\psi$.

The following lemma proves that unitaries $U\left(t_{1}, \ldots, t_{\tau} ; E\right)$ and $U\left(s_{1}, s_{2}, \ldots, s_{r} ; E\right)$ are close if their indices are sufficiently close.

Proposition 4.1.1 Let $1 \geq t_{1} \geq t_{2} \geq \cdots \geq t_{r} \geq 0$ and $1 \geq s_{1} \geq s_{2} \geq \cdots \geq$ $s_{r} \geq 0$ be two sequences of real numbers such that $\left|t_{i}-s_{i}\right|<\delta$, and $E$ be a positive integer. Then

$$
\left\|U\left(t_{1}, t_{2}, \ldots, t_{r} ; E\right)-U\left(s_{1}, s_{2}, \ldots, s_{r} ; E\right)\right\|<\pi \delta .
$$

Proof. As $\left|t_{i}-s_{i}\right|<\delta,\left\|u_{i, t_{i}, E}-u_{i, s_{i}, E}\right\|<\pi \delta$; since $u_{i, t_{i}, E}$ and $u_{j, t_{j}, E}$ act on orthogonal Hilbert spaces for $i \neq j$, the conclusion follows immediately.

Proposition 4.1.2 Let $\alpha=\operatorname{Std}\left(s_{1}, \ldots, s_{F}, E, Z\right)$ and $\beta=\operatorname{Std}\left(t_{1}, \ldots, t_{F}, E, Z\right)$ be represented on the same Hilbert space. Let $p_{i}$ and $q_{i}$ denote the support projections of $e_{s_{i}}$ and $e_{t_{i}}$ of $\alpha$ and $\beta$ respectively. If $s_{i}=t_{i}$ then $p_{i}=q_{i}$. Furthermore, there exists a unitary $U$ such that $U p_{i} U^{*}=q_{i}$ for all $i \in\{1, \ldots, F\}$ and $\|\mathrm{Id}-U\| \leq \pi \max \left\{\left|s_{i}-t_{i}\right|\right\}_{1}^{F}$.

Proof. We believe that the proof of the proposition is clear from the construction of standard maps, but will nevertheless try and provide further explanation. There are three cases. 
Case 1: $Z<a_{1}-E$.

By Case 2 of the construction given for maps in Section 4.1 standard form,

$$
\alpha=e_{s_{Z+1}} \oplus \cdots \oplus e_{s_{F}} \oplus R_{1}
$$

and

$$
\beta=e_{t_{Z+1}} \oplus \cdots \oplus e_{t_{F}} \oplus R_{2}
$$

where $R_{1}$ and $R_{2}$ are defined as follows:

$$
\begin{aligned}
& R_{1}=\operatorname{Ad}_{U^{\prime}\left(s_{1}, \ldots, s_{Z} ; E\right)}\left(e_{s_{1}} \oplus \cdots \oplus e_{s_{Z}} \bigoplus_{1}^{E} e_{\infty} \bigoplus_{1}^{z} 0\right) \\
& R_{2}=\operatorname{Ad}_{U^{\prime}\left(t_{1}, \ldots, t_{Z} ; E\right)}\left(e_{t_{1}} \oplus \cdots \oplus e_{t_{z}} \bigoplus_{1}^{E} e_{\infty} \bigoplus_{1}^{z} 0\right) .
\end{aligned}
$$

For $i \geq Z+1$ it is clear from the above description of the maps that the support projection of $e_{s_{i}}$ is $e_{t_{i}}$ are the same.

For $i<Z+1$ we need to examine the structure of the unitaries $U(\bullet, \ldots, \bullet)$ used to define the maps $R_{i}$. The unitaries $U^{\prime}\left(s_{1}, \ldots, s_{Z} ; E\right)$ and $U^{\prime}\left(t_{1}, \ldots, t_{Z} ; E\right)$ are defined as a product of "elementary unitaries", $u_{i, s_{i}, E}^{\prime}$ and $u_{i, t_{i}, E}^{\prime}$ by equation (4.9). Each of the elementary unitaries $u_{i, \bullet, E}^{\prime}$ act as the identity except on the subspace spanned by $\left\{\xi_{m+\left(a_{1}+1\right) i}, \xi_{m+Z\left(a_{1}+1\right)+E+i}\right\}$ where $m+Z\left(a_{1}+1\right)+E+Z=\operatorname{dim} R_{1}=\operatorname{dim} R_{2}$. As the dimension of the subspace on which $R_{1}$ is canonically represented is equal to $Z\left(a_{1}+1\right)+E+Z$, $m=0$. Therefore, the unitary $u_{i, \bullet, E}^{\prime}$ acts (non-trivially) only on the subrepresentation $e_{s_{i}}$ and the $i^{\prime}$ th zero of the direct summand $\bigoplus_{1}^{Z} 0$, for $1 \leq i \leq Z$. If $s_{i}=t_{i}$ then the unitaries $u_{i, s_{i}, E}^{\prime}$ and $u_{i, t_{i}, E}^{\prime}$ will by definition be equal, and hence the support projections of $e_{s_{i}}$ and $e_{t_{i}}$ will also be equal. In general, we 
know that

$$
\left\|u_{i, s_{i}, E}^{\prime}-u_{i, t_{i}, E}^{\prime}\right\| \leq \pi\left|s_{i}-t_{i}\right| .
$$

Let $U=U^{\prime}\left(t_{1}, \ldots, t_{Z} ; E\right)\left(U^{\prime}\left(t_{1}, \ldots, t_{Z} ; E\right)\right)^{*}$. Then $U p_{i} U^{*}=q_{i}$, where $p_{i}$ and $q_{i}$ are the support projections of the subrepresentations $e_{s_{i}}$ and $e_{t_{i}}$ respectively, for $1 \leq i \leq Z$. As the unitary $U$ can be written as a product of elementary matrices, $\|U-\mathrm{Id}\| \leq \pi \max \left\{\left|s_{i}-t_{i}\right|\right\}_{i=1}^{Z}$.

Case 2: $Z \geq a_{1}-E$.

There are two subcases.

Subcase 2.1: $k_{Z} \leq k$.

If $k_{Z} \leq k$, and $r_{Z} \leq r$ if $k_{Z}=k$ then by Subcase 3.1 of Section 4.1, the maps are defined as follows: $\alpha=\iota_{r_{Z}, r_{Z}}^{\prime}\left(\iota_{a_{1}}\right)^{k_{Z}}\left(L_{1}\right)$ and $\beta=\iota_{r_{Z}, r_{Z}}^{\prime}\left(\iota_{a_{1}}\right)^{k_{Z}}\left(L_{2}\right)$. If $k_{Z}=k$ and $r_{Z}>r$ then by Subcase 3.2 of Section 4.1, the maps are defined as $\alpha=\iota_{r, r_{Z}}^{\prime}\left(\iota_{a_{1}}\right)^{k_{Z}}\left(L_{1}\right)$ and $\beta=\iota_{r, r_{Z}}^{\prime}\left(\iota_{a_{1}}\right)^{k_{Z}}\left(L_{2}\right)$. In both cases, as $k_{Z} \leq k$, by Remark 4.1.3 we can give an explicit construction for $\left(\iota_{a_{1}}\right)^{k_{Z}}\left(L_{i}\right)$ :

$$
\left(\iota_{a_{1}}\right)^{k_{Z}}\left(L_{1}\right)=e_{s_{a_{1}-E+k_{Z}\left(a_{1}\right)+1}} \oplus \cdots \oplus e_{s_{F}} \oplus R_{1} \bigoplus_{j=0}^{k_{Z}-1} \mathcal{S}_{1, j}
$$

and

$$
\left(\iota_{a_{1}}\right)^{k_{Z}}\left(L_{2}\right)=e_{t_{a_{1}-E+k_{Z}\left(a_{1}\right)+1}} \oplus \cdots \oplus e_{t_{F}} \oplus R_{2} \bigoplus_{j=0}^{k_{Z}-1} \mathcal{S}_{2, j}
$$

where

$$
\begin{aligned}
& R_{1}=\operatorname{Ad}_{U^{\prime}\left(s_{1}, \ldots, s_{Z} ; E\right)}\left(e_{s_{1}} \oplus \cdots \oplus e_{s_{Z}} \bigoplus_{1}^{E} e_{\infty} \bigoplus_{1}^{Z} 0\right) \\
& R_{2}=\operatorname{Ad}_{U^{\prime}\left(t_{1}, \ldots, t_{Z} ; E\right)}\left(e_{t_{1}} \oplus \cdots \oplus e_{t_{Z}} \bigoplus_{1}^{E} e_{\infty} \bigoplus_{1}^{Z} 0\right)
\end{aligned}
$$




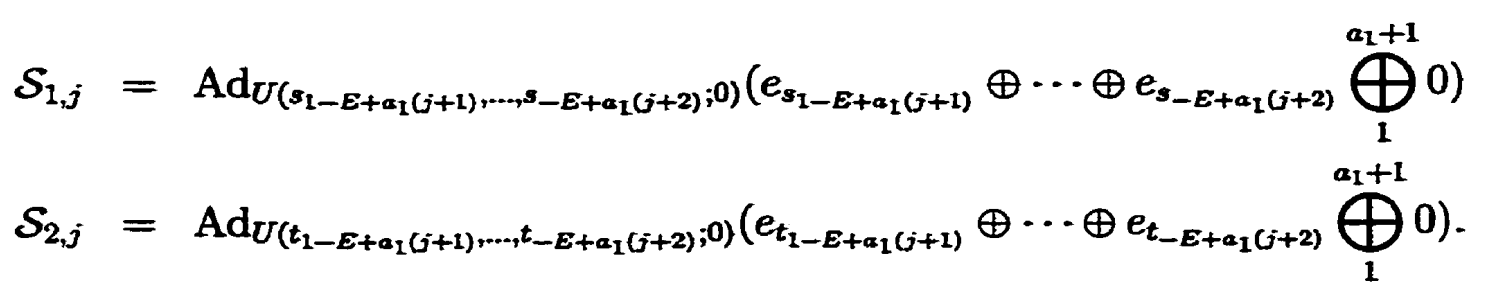

From the construction given above, it is clear that for $i \geq a_{1}-E+k_{Z} a_{1}+1$ the support projection of $e_{s_{i}}$ and $e_{t_{i}}$, viewed as subrepresentations of $c_{a_{1}}^{k_{Z}}\left(L_{1}\right)$ and $\iota_{\alpha_{1}}^{k_{Z}}\left(L_{2}\right)$ respectively, are equal. The arguments given in Case 1 for $R_{1}$ and $R_{2}$ apply equally well to $R_{1}, R_{2}, \mathcal{S}_{1, j}$ and $\mathcal{S}_{2, j}$. For each subrepresentation $e_{s_{i}}$ and $e_{t_{i}}$ of $R_{1}$ and $R_{2}$ (or $\mathcal{S}_{1, j}$ and $\mathcal{S}_{2, j}$ ) if $s_{i}=t_{i}$ then their respective support projections are equal. As in Case 1 we can find a unitary $U$ such that $U p_{i} U^{*}=q_{i}$ and $\|U-\operatorname{Id}\| \leq \pi \max \left\{\left|s_{i}-t_{i}\right|\right\}_{i=1}^{a_{1}-E+k_{2} a_{1}+1}$, for $1 \leq i \leq$ $a_{1}-E+k_{Z} a_{1}+1$.

The action of $\iota_{r Z, r Z}^{\prime}$ or $\iota_{r, r z}^{\prime}$ on $\iota_{a_{1}}^{k_{Z}}\left(L_{1}\right)$ and $\iota_{a_{1}}^{k_{Z}}\left(L_{2}\right)$ is implemented by a unitary shift $\left(V^{*}\right)^{r z}$ and a unitary twist of the form $U^{\prime}(\bullet, \ldots, \bullet)$. The unitary $U^{\prime}(\bullet, \ldots, \bullet)$ can be decomposed into a product of elementary unitaries. As argued in Case 1, the elementary unitaries agree if their indices are equal, and are otherwise supported on a subrepresentation of the corresponding index. Furthermore, the difference between two elementary unitaries is bounded by the difference between their indices.

Subcase 2.2: $k_{Z}>k$.

By Subcase 3.3 of Section 4.1,

$$
\alpha=\iota_{0, r z}^{\prime}\left(\iota_{0}\right)^{k z-k-1} \iota_{r}\left(\iota_{a_{1}}\right)^{k}\left(L_{1}\right)
$$

and

$$
\beta=\iota_{0, r Z}^{\prime}\left(\iota_{0}\right)^{k_{Z}-k-1} \iota_{r}\left(\iota_{a_{1}}\right)^{k}\left(L_{2}\right)
$$


We can apply Subcase 2.1 to the maps $\iota_{\tau}\left(\iota_{\alpha_{1}}\right)^{k}\left(L_{1}\right)$ and $\iota_{\tau}\left(\iota_{\alpha_{1}}\right)^{k}\left(L_{2}\right)$ to conclude that the support projections of the subrepresentations $e_{s_{i}}$ and $e_{t_{i}}$ are equal when $s_{i}=t_{i}$, and can in general be conjugated to each other by a unitary whose distance to the identity is bounded by the maximum of the difference of $s_{i}$ and $t_{i}$ for $i=1, \ldots, F$. As the map $\iota_{0,5 Z}^{\prime}\left(\iota_{0}\right)^{k_{Z}-k-1}$ has the same unitary action on $\iota_{\tau}\left(\iota_{a_{1}}\right)^{k}\left(L_{1}\right)$ and $\iota_{\tau}\left(\iota_{a_{1}}\right)^{k}\left(L_{2}\right)$, the conclusions outlined above also hold true for the maps $\alpha$ and $\beta$. This completes the proof of the proposition.

\subsection{Comparing maps in standard form}

This section will be devoted entirely to proving the following theorem about standard maps.

Lemma 4.2.1 Let $A_{1}$ and $A_{2}$ be building blocks and $h$ be the canonical selfadjoint element of $A_{1}$. Let $\delta$ be a real number such that $\varepsilon / 2 \pi>\delta \geq 0$. Let $F$ be a subset of $A_{1}$ such that if $\left|t_{0}-t_{1}\right|<\delta$ then $\left\|f\left(t_{0}\right)-f\left(t_{1}\right)\right\|<\varepsilon$ for all $f \in F$. Let $\phi$ and $\psi$ be maps from $A_{1}$ to $A_{2}$ such that the eigenvalues of $\phi(h)(t)$ and $\psi(h)(t)$ can be matched to within $\delta$, and such that there are at least $a_{1}+1$ distinct such values, for each $t \in[0,1]$. Then

$$
\left\|\phi^{\prime}(f)-\psi^{\prime}(f)\right\|<7 \varepsilon \text { for all } f \in F \text {. }
$$

Proof. As $\phi^{\prime}(f)$ and $\psi^{\prime}(f)$ are matrix valued functions over the unit interval, it is sufficient to show that $\phi^{\prime}(f)(s)$ and $\psi^{\prime}(f)(s)$ are within $\varepsilon$ for all $s \in[0,1]$ and $f \in F$. Let $s$ be any point in the interval $[0,1]$, then after grouping representations together by applying Lemma 4.1.1, there exists 
unique sequences $1 \geq s_{1} \geq s_{2} \cdots \geq s_{F_{1}} \geq 0$ and $1 \geq t_{1} \geq t_{2} \cdots \geq t_{F_{2}} \geq 0$, unique positive integers $E_{1}, E_{2}, Z_{1}$, and $Z_{2}$ satisfying conditions (i) and (ii) of the lemma such that $f_{s} \phi$ and $f_{s} \psi$ are unitarily equivalent to $e_{s_{1}} \oplus e_{s_{2}} \oplus$ $\cdots \oplus e_{s_{F_{1}}} \bigoplus_{1}^{E_{1}} e_{\infty} \bigoplus_{1}^{Z_{1}} 0$ and $e_{t_{1}} \oplus e_{t_{2}} \oplus \cdots \oplus e_{t_{F_{2}}} \bigoplus_{1}^{E_{2}} e_{\infty} \bigoplus_{1}^{Z_{2}} 0$ respectively.

Define integers $k_{i}, r_{i}, k_{Z_{i}}$ and $r_{Z_{i}}$ for $i=1,2$ as follows:

$$
\begin{aligned}
F_{i}-\left(a_{1}-E_{i}\right) & =k_{i} a_{1}+r_{i} \\
Z_{i}-\left(a_{1}+1-E_{i}\right) & =k_{Z_{i}}\left(a_{1}+1\right)+r_{Z_{i}}
\end{aligned}
$$

where $0 \leq r_{i}<a_{1}, 0 \leq r_{Z_{i}}<a_{1}+1$ and $k_{i}, k_{Z_{i}}$ are positive. There are four cases. The strategy is the same in all four cases; we begin with $f_{s} \psi^{\prime}(f)$ and then compare it with a sequence of similar expressions (where terms in the sequence are within $\varepsilon$ of neighbouring terms in the sequence on the finite set $F)$, until we arrive at the last term in the sequence, $f_{s} \phi^{\prime}(f)$.

Case 1: $Z_{1}<a_{1}+1-E_{1}$ and $Z_{2}<a_{1}+1-E_{2}$.

Without loss of generality it is possible to assume that $Z_{1} \leq Z_{2}$. Let $Z=Z_{2}-Z_{1}$. By counting the dimensions of the representations,

$$
\operatorname{dim}\left(D_{2}\right)=\left(a_{1}+1\right) F_{1}+E_{1}+Z_{1}=\left(a_{1}+1\right) F_{2}+E_{2}+Z_{2} .
$$

As $E_{i}+Z_{i}<a_{1}+1$ for $i=1,2$,

$$
E_{1}+Z_{1}=E_{2}+Z_{2} \text { and } F_{1}=F_{2}
$$

Let $F=F_{1}=F_{2}$ and $Z=Z_{2}-Z_{1}=E_{1}-E_{2}$. The eigenvalues at $s$ of $\phi(h)$ and $\psi(h)$ in ascending order, with multiplicity are:

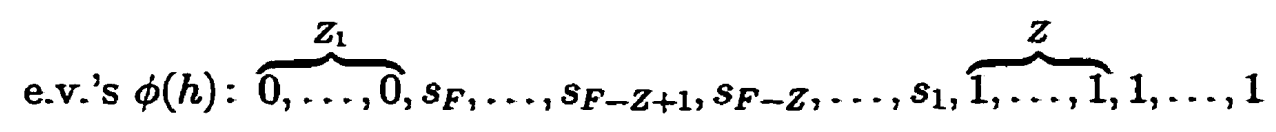




$$
\text { e.v.'s } \psi(h): \underbrace{0, \ldots, 0}_{Z_{1}}, \underbrace{0, \ldots, 0}_{Z}, t_{F}, \ldots, t_{Z+1}, t_{Z}, \ldots, t_{1}, 1, \ldots, 1 \text {. }
$$

As the eigenvalues can be matched to within $\delta$, comparing the above two lists the following relations are evident:

$$
\begin{aligned}
\left|s_{i}-0\right| & <\delta \text { for } i=F, F-1, \ldots, F-Z+1 \\
\left|t_{i}-1\right| & <\delta \text { for } i=1,2, \ldots, Z \\
\left|s_{i-Z}-t_{i}\right| & <\delta \text { for } i=Z+1, Z+2, \ldots F .
\end{aligned}
$$

In terms of evaluation maps,

$$
\begin{aligned}
\left|e_{s_{i}}(f)-e_{0}(f)\right| & <\delta \text { for } i=F, F-1, \ldots, F-Z+1 \\
\left|e_{t_{i}}(f)-e_{1}(f)\right| & <\delta \text { for } i=1,2, \ldots, Z \\
\left|e_{s_{i}-Z}(f)-e_{t_{i}}(f)\right| & <\delta \text { for } i=Z+1, Z+2, \ldots F
\end{aligned}
$$

for $f \in F$. The above relations will be used repeatedly to make the following estimates:

$$
\begin{aligned}
& f_{s} \psi^{\prime}(f) \\
& =\bigoplus_{i=Z_{2}+1}^{F} e_{t_{i}} \oplus R_{2}(f) \\
& =\bigoplus_{i=Z_{2}+1}^{F} e_{t_{i}} \oplus A d_{U^{\prime}\left(t_{1}, \ldots, t_{Z_{2}} ; E_{2}\right)}\left(e_{t_{1}} \oplus \cdots \oplus e_{t_{z_{2}}} \bigoplus_{1}^{E_{2}} e_{\infty} \bigoplus_{1}^{Z_{2}} 0\right)(f) \\
& \sim_{2 \pi \delta} \bigoplus_{i=Z_{2}+1}^{F} e_{t_{i}} \oplus A d_{U}(\underbrace{\left.1, \ldots, 1, s_{1}, \ldots s_{Z_{1}} ; E_{2}\right)}_{Z}\left(\bigoplus_{i=1}^{Z} e_{t_{i}} \bigoplus_{i=Z+1}^{Z_{2}} e_{t_{i}} \bigoplus_{1}^{E_{2}} e_{\infty} \bigoplus_{1}^{Z} 0 \bigoplus_{Z+1}^{Z_{2}} 0\right)(f) \\
& \sim_{\varepsilon} \quad \bigoplus_{i=Z_{2}+1}^{F} e_{t_{i}} \oplus A d_{U^{\prime}\left(1, \ldots, 1, s_{1}, \ldots, z_{1} ; E_{2}\right)}\left(\bigoplus_{i=1}^{Z} e_{1} \bigoplus_{i=1}^{Z_{1}} e_{s_{i}} \bigoplus_{1}^{E_{2}} e_{\infty} \bigoplus_{1}^{Z} 0 \bigoplus_{1}^{Z_{1}} 0\right)(f) \\
& =\bigoplus_{i=Z_{2}+1}^{F} e_{t_{i}} \oplus A d_{U^{\prime}\left(0, \ldots, 0, s_{1}, \ldots, z_{1} ; E_{2}\right)}\left(\bigoplus_{i=1}^{Z} e_{0} \bigoplus_{i=1}^{Z_{1}} e_{s_{i}} \bigoplus_{1}^{E_{2}} e_{\infty} \bigoplus_{1}^{Z} e_{\infty} \bigoplus_{1}^{Z_{1}} 0\right)(f)
\end{aligned}
$$




$$
\begin{aligned}
& =\bigoplus_{i=Z_{2}+1}^{F} e_{t_{i}} \bigoplus_{1}^{Z} e_{0} \bigoplus A d_{U^{\prime}\left(s_{1}, \ldots, s_{Z_{1}}, E_{2}\right)}\left(\bigoplus_{i=1}^{Z_{1}} e_{s_{i}} \bigoplus_{1}^{Z+E_{2}} e_{\infty} \bigoplus_{1}^{Z_{1}} 0\right)(f) \\
& =\bigoplus_{i=Z_{2}+1}^{F} e_{t_{i}} \bigoplus_{1}^{Z} e_{0} \oplus R_{1}(f) \\
& \sim_{\varepsilon} \bigoplus_{i=Z_{1}+1}^{F-Z} e_{s_{i}} \bigoplus_{F-Z+1}^{F} e_{s_{i}} \oplus R_{1}(f) \\
& =\bigoplus_{i=Z_{1}+1}^{F} e_{s_{i}} \oplus R_{1}(f) \\
& =f_{s} \phi^{\prime}(f)
\end{aligned}
$$

for all $f \in F$. By the above calculation, and noting that $2 \pi \delta<\varepsilon$,

$$
\left\|\phi^{\prime}(f)-\psi^{\prime}(f)\right\|<3 \varepsilon
$$

Case 2: $Z_{1}<a_{1}+1-E_{1}$ and $Z_{2} \geq a_{1}+1-E_{2}$.

To simplify the argument it is necessary to perturb $f_{s} \phi^{\prime}$ if $s_{i}=1$ for some value of $i$. The conclusion of Lemma 4 .1.1 ensures that $s_{i}=1$ for some value of $i$, only if $Z_{1}=0$. Suppose the $s_{i}=1$ for $i=1, \ldots, k$, then define $\phi^{\prime \prime}: A_{1} \rightarrow M_{m}(\mathbb{C})$ as follows:

$$
\phi^{\prime \prime}=e_{s_{1}^{\prime}} \oplus e_{s_{2}^{\prime}} \oplus \cdots \oplus e_{s_{F_{1}}^{\prime}} \bigoplus_{1}^{E_{1}} e_{\infty}
$$

where

$$
s_{i}^{\prime}=\left\{\begin{aligned}
\max \left(1-\delta, s_{k+1}\right) & \text { for } i=1, \ldots, k \\
s_{i} & \text { otherwise. }
\end{aligned}\right.
$$

From the construction of $\phi^{\prime \prime}$ it is clear that $s_{i}^{\prime} \neq 1$ for all $i$. As $\left|s_{i}-s_{i}^{\prime}\right|<\delta$, $\left\|e_{s_{i}}(f)-e_{s_{i}^{\prime}}(f)\right\|<\varepsilon$ for $f \in F$. It follows from the construction of $\phi^{\prime}$ that

$$
\left\|\phi^{\prime}(f)-\phi^{\prime \prime}(f)\right\|<\varepsilon \text { for } f \in F
$$


Therefore we can assume that $s_{i} \neq 1$ for all $i$, provided that an extra $\varepsilon$ is accounted for in the final degree of comparability for this case of the proof.

As in case one, by counting the dimensions of representations,

$$
\begin{aligned}
\left(a_{1}+1\right) F_{1}+Z_{1}+E_{1} & =\operatorname{dim}\left(D_{2}\right) \\
& =\left(a_{1}+1\right) F_{2}+Z_{2}+E_{2} \\
& =\left(a_{1}+1\right)\left(F_{2}+1\right)+Z_{2}-\left(a_{1}+1-E_{2}\right) \\
& =\left(a_{1}+1\right)\left(F_{2}+1\right)+k_{Z_{2}}\left(a_{1}+1\right)+r_{Z_{2}} \\
& =\left(a_{1}+1\right)\left(F_{2}+k_{Z_{2}}+1\right)+r_{Z_{2}} .
\end{aligned}
$$

(Recall that the positive integers $k_{Z_{2}}$ and $r_{Z_{2}}$ were define by the pair of equations (4.12).) Let $Z=Z_{2}-Z_{1}$. It follows from the above calculation and the assumption $Z_{1}+E_{1}<a_{1}+1$ that

$$
\begin{aligned}
r_{Z_{2}} & =Z_{1}+E_{1} \\
F_{1} & =F_{2}+k_{Z_{2}}+1 \\
Z & =E_{1}-E_{2}+\left(k_{Z_{2}}+1\right)\left(a_{1}+1\right) .
\end{aligned}
$$

Since $k_{Z_{2}} \geq 0$ and $\left|E_{1}-E_{2}\right| \leq a_{1}$ the last equation, above, guarantees that $Z>0$. The number of eigenvalues of $\phi^{\prime}(h)(s)$ with eigenvalue one is $a_{1} F_{1}+E_{1}$ since $e_{s_{i}^{r}} \neq 1$ for all $i$. Similarly, the number of eigenvalues of $\psi^{\prime}(h)(s)$ with eigenvalue one is $a_{1} F_{2}+E_{2}$. Their difference is

$$
a_{1}\left(F_{1}-F_{2}\right)+E_{1}-E_{2}=a_{1}\left(k_{Z_{2}}+1\right)+E_{1}-E_{2}
$$

The eigenvalues at $s$ of $\phi^{\prime}(h)$ and $\psi^{\prime}(h)$ in ascending order, with multiplicity are:

$$
\text { e.v.'s } \phi^{\prime}(h): \overbrace{0, \ldots, 0}^{Z_{1}}, s_{F_{1}}, \ldots, s_{F_{1}-Z+1}, s_{F_{1}-Z}, \ldots, s_{1}, \overbrace{1, \ldots, 1}^{l-1}, 1, \ldots, 1
$$




$$
\text { e.v.'s } \psi^{\prime}(h)=\underbrace{0, \ldots, 0}_{Z_{1}}, \underbrace{0, \ldots, 0}_{Z}, t_{F_{2}}, \ldots, t_{l_{2}} t_{l-1}, \ldots, t_{1}, 1, \ldots, 1
$$

where $l-1=a_{1}\left(k_{Z_{2}}+1\right)+E_{1}-E_{2}$. Comparing the above two lists, the following relations are evident and will be used repeatedly:

$$
\begin{aligned}
\left|s_{i}-0\right| & <\delta \text { for } i=F_{1}, F_{1}-1, \ldots, F_{1}-Z+1 \\
\left|t_{i}-1\right| & <\delta \text { for } i=1,2, \ldots, l-1 \\
\left|t_{l-1+i}-s_{i}\right| & <\delta \text { for } i=1,2, \ldots, F_{2}-(l-1) \\
F_{2}-l & =F_{1}-Z-1 .
\end{aligned}
$$

As in case one, the above relations guarantee corresponding relations between the evaluation maps on a finite set of elements $F$.

Proposition 4.2.1 $k_{2} \geq k_{Z_{2}}$.

Proof. Since $\left|t_{i}-1\right|<\delta$ for $a_{1}\left(k_{Z_{2}}+1\right)+E_{1}-E_{2}$ distinct values of $i$, $F_{2} \geq a_{1}\left(k_{Z_{2}}+1\right)+E_{1}-E_{2}$. Then $a_{1} k_{2}+r_{2}=F_{2}-\left(a_{1}-E_{2}\right) \geq a_{1} k_{Z_{2}}+E_{1}$, which can be rearranged as

$$
a_{1}\left(k_{2}-k_{Z_{2}}\right) \geq E_{1}-r_{2}
$$

Since $r_{2}<a_{1}$, it follows from the above inequality that $k_{2} \geq k_{Z_{2}}$. (The positive integers $k_{2}$ and $r_{2}$ were defined in (4.12).)

Following the arguments given in Remark 4.1.3, as $k_{Z_{2}} \leq k_{2}$ and $Z_{2} \geq$ $a_{1}+1-E_{2}$

$$
\iota_{a_{1}}^{k Z_{2}}\left(L_{2}\right)=e_{t_{a_{1}-E_{2}+k_{Z_{2}}\left(a_{1}\right)+1}} \oplus \cdots \oplus e_{t_{F_{2}}} \oplus R_{2} \bigoplus_{j=0}^{k_{Z_{2}}-1} \mathcal{S}_{j} .
$$


79

From the second equation in (4.17), $\left|t_{i}-1\right|<\delta$ for the first $l-1$ values of $i$; using this inequality we can make the following estimate for $R_{2}(f)$ :

$$
\begin{aligned}
R_{2}(f) & =A d_{U\left(t_{1}, \ldots, t_{a_{1}-E_{2}}-E_{2}\right)}\left(e_{t_{1}} \oplus \cdots \oplus e_{t_{a_{1}-E_{2}}} \bigoplus_{1}^{E_{2}} e_{\infty} \bigoplus_{1}^{a_{1}+1-E_{2}} 0\right)(f) \\
& \sim_{2 \pi \delta} \quad A_{U\left(1, \ldots, 1 ; E_{2}\right)}\left(e_{t_{1}} \oplus \cdots \oplus e_{t_{\alpha_{1}-E_{2}}} \bigoplus_{1}^{E_{2}} e_{\infty} \bigoplus_{1}^{a_{1}+1-E_{2}} 0\right)(f) \\
& \sim_{\varepsilon} \quad A d_{U\left(1, \ldots, 1 ; E_{2}\right)}\left(e_{1} \oplus \cdots \oplus e_{1} \bigoplus_{1}^{E_{2}} e_{\infty} \bigoplus_{1}^{a_{1}+1-E_{2}} 0\right)(f) \\
& =\bigoplus_{1}^{a_{1}+1-E_{2}} e_{0}(f)
\end{aligned}
$$

for all $f \in F$. For $j \leq k_{Z_{2}}-1$ and $i \leq a_{1}+1$,

$$
\begin{aligned}
a_{1}-E_{2}+j a_{1}+i & \leq a_{1}-E_{2}+\left(k_{Z_{2}}-1\right) a_{1}+\left(a_{1}+1\right) \\
& \leq l-1 .
\end{aligned}
$$

Therefore, by the second equation of (4.17), $\left|t_{a_{1}-E_{2}+j a_{1}+i}-1\right|<\delta$ for $1 \leq$ $i \leq a_{1}+1$ and $j \leq k_{Z_{2}}-1$. Using this inequality a similar estimate can be found for $\mathcal{S}_{j}(f)$ as was found for $\boldsymbol{R}_{2}(f)$ :

$$
\begin{aligned}
& \mathcal{S}_{j}(f)=A d_{U\left(t_{a_{1}-E_{2}+j a_{1}+1}, \ldots, t_{a_{1}-E_{2}+j a_{1}+\left(a_{1}+1\right)}\right.}\left(e_{t_{a_{1}-E_{2}+j a_{1}+1}} \oplus \cdots \oplus\right. \\
&\left.e_{t_{a_{1}-E_{2}+j a_{1}+\left(a_{1}+1\right)}} \bigoplus_{1}^{a_{1}+1} 0\right)(f) \\
& \sim_{2 \pi \delta} \quad A d_{U(1, \ldots, 1)}\left(e_{t_{a_{1}}-E_{2}+j a_{1}+1} \oplus \cdots \oplus e_{t_{a_{1}-E_{2}+j a_{1}+\left(a_{1}+1\right)}} \bigoplus_{1}^{a_{1}+1} 0\right)(f) \\
& \sim_{\varepsilon} \quad A d_{U(1, \ldots, 1)}\left(e_{1} \oplus \cdots \oplus e_{1} \oplus_{1}^{a_{1}+1} 0\right)(f) \\
&=\bigoplus_{1}^{a_{1}+1} e_{0}(f)
\end{aligned}
$$


80

for all $f \in F$. Using the above approximations to $R_{2}$ and $\mathcal{S}_{j}$ and noting that $2 \pi \delta<\varepsilon$,

$$
\begin{aligned}
& \sim_{c_{1} \varepsilon}^{k_{Z_{2}}}\left(L_{2}\right)(f) \\
& e_{t_{a_{1}-E_{2}+k_{Z_{2}}\left(a_{1}\right)+1} \oplus \cdots \oplus e_{t_{F_{2}}}} \bigoplus_{1}^{a_{1}+1-E_{2}} e_{0} \bigoplus_{j=0}^{k_{Z_{2}}-1}\left(\bigoplus_{1}^{a_{1}+1} e_{0}\right)(f) \\
& =e_{t_{l-E_{1}}} \oplus \cdots \oplus e_{t_{l-1}} \oplus e_{t_{l}} \oplus \cdots \oplus e_{t_{F_{2}}} \bigoplus_{1}^{\left(a_{1}+1\right)\left(k_{z_{2}}+1\right)-E_{2}} e_{0}(f) \\
& \sim_{\varepsilon} \bigoplus_{1}^{E_{1}} e_{1} \oplus e_{t_{l}} \oplus \cdots \oplus e_{t_{F_{2}}} \bigoplus_{1}^{\left(a_{1}+1\right)\left(k_{Z_{2}}+1\right)-E_{2}} e_{0}(f) .
\end{aligned}
$$

Therefore,

$$
i_{a_{1}}^{k_{Z_{2}}}\left(L_{2}\right)(f) \sim \sim_{3 \epsilon} \bigoplus_{1}^{E_{1}} e_{1} \oplus e_{t_{l}} \oplus \cdots \oplus e_{t_{F_{2}}} \bigoplus_{1}^{\left(a_{1}+1\right)\left(k_{Z_{2}}+1\right)-E_{2}} e_{0}(f)
$$

There are two subcases.

Subcase 2.1: $k_{Z_{2}} \leq k_{2}$, and $r_{Z_{2}} \leq r_{2}$ if $k_{Z_{2}}=k_{2}$.

Under these assumptions, and using the first of the three equations in (4.15) for the last step,

$$
\begin{aligned}
F_{2}-l+1 & =\left(a_{1}-E_{2}\right)+a_{1} k_{2}+r_{2}-\left(a_{1}\left(k_{Z_{2}}+1\right)+E_{1}-E_{2}\right) \\
& =a_{1}\left(k_{2}-k_{Z_{2}}\right)+r_{2}-E_{1} \\
& \geq r_{Z_{2}}-E_{1} \\
& =Z_{1} .
\end{aligned}
$$

We will use the above calculation and equations (4.15) and (4.17) to simplify the expression given by applying $\iota_{r z_{2}, r z_{2}}^{\prime}$ to equation (4.20);

$$
f_{s} \psi^{\prime}(f)
$$


81

$$
\begin{aligned}
& =\iota_{r Z_{2}, r Z_{2}}^{\prime} \iota_{a_{1}}^{\iota_{Z_{2}}}\left(L_{2}\right)(f) \\
& \sim_{3 \varepsilon} \quad \iota_{Z_{1}+E_{1}, Z_{1}+E_{1}}^{\prime}\left(\bigoplus_{1}^{E_{1}} e_{1} \oplus e_{t_{l}} \oplus \cdots \oplus e_{t_{F_{2}}}\right. \\
& \left(a_{1}+1\right)\left(k_{Z_{2}}+1\right)-E_{2} \\
& \left(a_{1}+1\right)\left(k_{Z_{2}}+1\right)-E_{2} \\
& \sim_{3 \varepsilon} \quad e_{t_{t+Z_{1}}} \oplus \cdots \oplus e_{t_{F_{2}}} \\
& \bigoplus \quad e_{0} \\
& \oplus \mathrm{Ad}_{U^{\prime}}(\underbrace{1, \ldots, 1}_{E_{1}}, t_{t}, \ldots, t_{t+Z_{1}-1})\left(\bigoplus_{1}^{E_{1}} e_{1} \oplus e_{t_{t}} \oplus \cdots \oplus e_{t_{t+z_{1}-1}} \bigoplus_{1}^{E_{1}+Z_{1}} 0\right)(f) \\
& \left(a_{1}+1\right)\left(k_{Z_{2}}+1\right)-E_{2} \\
& =e_{t_{l+z_{1}}} \oplus \cdots \oplus e_{t_{f_{2}}} \\
& \bigoplus \quad e_{0} \\
& \bigoplus_{1}^{E_{1}} e_{o} \oplus \operatorname{Ad}_{U^{\prime}\left(t_{t}, \ldots, t_{t}+z_{1}-1\right)}\left(e_{t_{t}} \oplus \cdots \oplus e_{t_{t+z_{1}-1}} \bigoplus_{1}^{E_{1}} e_{\infty} \bigoplus_{1}^{Z_{1}} 0\right)(f) \\
& \left(a_{1}+1\right)\left(k_{Z_{2}}+1\right)-E_{2}+E_{2} \\
& \sim_{2 \varepsilon} \quad e_{s z_{1}+1} \oplus \cdots \oplus e_{s_{F_{1}-Z}} \\
& \bigoplus \quad e_{0} \\
& \oplus \operatorname{Ad}_{U^{\prime}\left(s_{1}, \ldots, s_{Z_{1}} ; E_{1}\right)}\left(e_{s_{1}} \oplus \cdots \oplus e_{s_{Z_{1}}} \bigoplus_{1}^{E_{1}} e_{\infty} \bigoplus_{1}^{Z_{1}} 0\right)(f) \\
& \left(a_{1}+1\right)\left(k_{z_{2}}+1\right)-E_{2}+E_{1} \\
& =e_{s_{Z_{1}+1}} \oplus \cdots \oplus e_{s_{F_{1}-z}} \\
& e_{0} \oplus R_{1}(f)
\end{aligned}
$$

Since $\left|s_{i}-0\right|<\delta$ for the $Z$ values of the index, $i=F_{1}-Z+1, \ldots F_{1}$, and noting that $Z=\left(k_{Z_{2}}+1\right)\left(a_{1}+1\right)+E_{1}-E_{2}$, the above expression is approximately within $\varepsilon$ of

$$
e_{s_{Z_{1}+1}} \oplus \cdots \oplus e_{s_{F_{1}-z}} \oplus e_{s_{F_{1}-z+1}} \oplus \cdots \oplus e_{s_{F_{1}}} \oplus R_{1}(f)=f_{s} \phi^{\prime}(f)
$$

on the finite set $F$. Therefore, from the above estimates, for $f \in F$,

$$
\left\|f_{s} \psi^{\prime}(f)-f_{s} \phi^{\prime}(f)\right\|<6 \varepsilon
$$

Subcase 2.2: $k_{Z_{2}}=k_{2}$ and $r_{Z_{2}}>r_{2}$. 
82

Under these assumptions, and noting the similar calculation made in (4.21),

$$
\begin{aligned}
F_{2}-l+1 & =a_{1}\left(k_{2}-k_{Z_{2}}\right)+r_{2}-E_{1} \\
& =r_{2}-E_{1} \\
& \leq r_{Z_{2}}-E_{1} \\
& =Z_{1} .
\end{aligned}
$$

Then applying $\iota_{r_{2}, r_{z_{2}}}^{\prime}$ to equation (4.20),

$$
\begin{aligned}
& f_{s} \psi^{\prime}(f) \\
& =\iota_{r_{2}, r_{2}}^{\prime}{ }^{i \ell_{a_{1}}}\left(L_{2}\right)(f) \\
& \sim_{3 \varepsilon} \quad \iota_{F_{2}-l+1+E_{1}, Z_{1}+E_{1}}^{\prime}\left(\bigoplus_{1}^{E_{1}} e_{1} \oplus e_{t_{l}} \oplus \cdots \oplus e_{t_{F_{2}}} \bigoplus_{1}^{\left(a_{1}+1\right)\left(k_{Z_{2}}+1\right)-E_{2}} e_{0}\right)(f) \\
& \left(a_{1}+1\right)\left(k_{Z_{2}}+1\right)-E_{2}-\left(Z_{1}-\left(F_{2}-l+1\right)\right) \\
& \sim_{3 \varepsilon} \quad \bigoplus_{1} \quad e_{0} \oplus \operatorname{Ad}_{U^{\prime}}(\underbrace{1, \ldots, 1}_{E_{1}}, \underbrace{t_{l}, \ldots, t_{F_{2}}}_{F_{2}-t+1}, \underbrace{0, \ldots, 0}_{z_{1}-\left(F_{2}-l+1\right)}) \\
& \left(\bigoplus_{1}^{E_{1}} e_{1} \oplus e_{t_{t}} \oplus \cdots \oplus e_{t_{F_{2}}} \bigoplus_{1}^{Z_{1}-\left(F_{2}-l+1\right)} e_{0} \bigoplus_{1}^{E_{1}+Z_{1}} 0\right)(f) \\
& = \\
& \left(a_{1}+1\right)\left(k_{Z_{2}}+1\right)-E_{2}-\left(Z_{1}-\left(F_{2}-l+1\right)\right) \\
& e_{0} \bigoplus_{1}^{E_{1}} e_{0} \oplus \operatorname{Ad}_{U^{\prime}\left(t_{t}, \ldots, t_{F_{2}}, 0, \ldots, 0_{F} E_{1}\right)} \\
& \left(e_{t_{l}} \oplus \cdots \oplus e_{t_{F_{2}}} \bigoplus_{1}^{Z_{1}-\left(F_{2}-l+1\right)} e_{0} \bigoplus_{1}^{E_{1}} e_{\infty} \bigoplus_{1}^{Z_{1}} 0\right)(f) \\
& \sim_{2 \varepsilon} \bigoplus_{1}^{Z-\left(Z_{1}-\left(F_{2}-l+1\right)\right)} e_{0} \oplus \operatorname{Ad}_{U^{\prime}\left(s_{1}, \ldots, s_{Z_{1}} ; E_{1}\right)}\left(e_{s_{1}} \oplus \cdots \oplus e_{s_{Z_{1}}} \bigoplus_{1}^{E_{1}} e_{\infty} \bigoplus_{1}^{Z_{1}} 0\right)(f) \\
& =\bigoplus_{1}^{F_{1}-Z_{1}} e_{0} \oplus R_{1}(f) \\
& \sim_{\varepsilon} \quad e_{s_{Z_{1}+1}} \oplus \cdots \oplus e_{s_{F_{1}}} \oplus R_{1}(f)
\end{aligned}
$$




$$
=f_{s} \phi^{\prime}(f)
$$

From the above estimates, for $f \in F$,

$$
\left\|f_{s} \psi^{\prime}(f)-f_{s} \phi^{\prime}(f)\right\|<6 \varepsilon .
$$

By Proposition 4.2.1, Subcases 2.1 and 2.2 are exhaustive for Subcase 2. By the estimates made in the two subcases, for all $f \in F$,

$$
\left\|f_{s} \psi^{\prime}(f)-f_{s} \phi^{\prime}(f)\right\|<6 \varepsilon \text {. }
$$

Case 3: $Z_{1}=a_{1}+1-E_{1}$ and $Z_{2} \geq a_{1}+1-E_{2}$.

Without loss of generality assume that if $Z_{2}=a_{1}+1-E_{2}$ then $E_{1} \geq E_{2}$. By counting the dimensions of the representations at $s$,

$$
\begin{aligned}
\left(a_{1}+1\right) F_{1}+Z_{1}+E_{1} & =\left(a_{1}+1\right)\left(F_{1}+1\right) \\
& =\left(a_{1}+1\right) F_{2}+Z_{2}+E_{2} \\
& =\left(a_{1}+1\right)\left(F_{2}+k_{Z_{2}}+1\right)+r_{Z_{2}} .
\end{aligned}
$$

Let $Z=Z_{2}-Z_{1}$. It follows from the above calculation that

$$
\begin{aligned}
r_{Z_{2}} & =0 \\
F_{1} & =F_{2}+k_{Z_{2}} \\
Z & =k_{Z_{2}}\left(a_{1}+1\right)+E_{1}-E_{2} .
\end{aligned}
$$

$Z \geq 0$ since by assumption either $k_{Z_{2}} \geq 0$, or if $k_{Z_{2}}=0$ then $E_{1} \geq E_{2}$. At $s$, $\phi^{\prime}(h)$ has $a_{1} F_{1}+E_{1}-\left(a_{1} F_{2}+E_{2}\right)=a_{1} k_{Z_{2}}+E_{1}-E_{2}$ more eigenvalues equal to one than $\psi^{\prime}(h)$. The eigenvalues at $s$ of $\phi^{\prime}(h)$ and $\psi^{\prime}(h)$ listed in ascending order, with multiplicity are:

$$
\text { e.v.'s } \phi^{\prime}(h): \overbrace{0, \ldots, 0}^{Z_{1}}, s_{F_{1}}, \ldots, s_{F_{1}-Z+1}, s_{F_{1}-Z}, \ldots, s_{1}, \overbrace{1, \ldots, 1}^{l-1}, 1, \ldots, 1(
$$




$$
\text { e.v.'s } \psi^{\prime}(h)=\underbrace{0, \ldots, 0}_{Z_{1}}, \underbrace{0, \ldots, 0}_{Z}, t_{F_{2}}, \ldots, t_{l}, t_{l-1}, \ldots, t_{1}, 1, \ldots, 1(4
$$

where $l-1=a_{1} k_{Z_{2}}+E_{1}-E_{2}$. (The two lists of eigenvalues, above are the same as in Case 2, only the value of $l$ differs by a constant.) As in Case 2, comparing the above two lists, it's possible to derive the same set of equations as in (4.17), but with the new value of $l$ defined above.

Proposition 4.2.2 $k_{2} \geq k_{Z_{2}}-1$

Proof. The argument that follows is very similar to that given in the proof of Proposition 4.2.1. As $\left|t_{i}-1\right|<\delta$ for at least $a_{1} k_{Z_{2}}+E_{1}-E_{2}$ values of $i$, by examining the list of eigenvalues it is clear that $F_{2} \geq a_{1} k_{Z_{2}}+E_{1}-E_{2}$. Then $a_{1} k_{2}+r_{2}=F_{2}-\left(a_{1}-E_{2}\right) \geq a_{1}\left(k_{Z_{2}}-1\right)+E_{1}$, which can be rearranged as

$$
a_{1}\left(k_{2}-k_{Z_{2}}+1\right) \geq E_{1}-r_{2}
$$

Since $r_{2}<a_{1}$, it follows from the above inequality that $k_{2} \geq k_{Z_{2}}-1$.

As calculated in Case 2, by equation (4.18),

$$
R_{2}(f) \sim_{2 \varepsilon} \oplus_{1}^{a_{1}+1-E_{2}} e_{0}(f)
$$

For $j \leq k_{Z_{2}}-2$ and $1 \leq i \leq a_{1}+1$,

$$
\begin{aligned}
a_{1}-E_{2}+j a_{1}+i & \leq a_{1}-E_{2}+\left(k_{Z_{2}}-2\right) a_{1}+\left(a_{1}+1\right) \\
& \leq l_{1} .
\end{aligned}
$$

Therefore, by equation (4.19) of Case 2,

$$
\mathcal{S}_{j}(f) \sim_{2 \varepsilon} \oplus_{1}^{a_{1}+1} e_{0}(f) \text { for } j \leq k_{Z_{2}}-2
$$


85

Subcase 3.1: $k_{2} \geq k_{Z_{2}}$.

Noting that $r_{Z_{2}}=0$, and using the calculation given in Remark 4.1.3 to simplify the expression for $f_{s} \psi^{\prime}$ :

$$
\begin{aligned}
f_{s} \psi^{\prime} & =c_{a_{1}}^{k_{Z_{2}}}\left(L_{2}\right) \\
& =e_{t_{a_{1}-E_{2}+k_{Z_{2}} a_{1}+1}} \oplus \cdots \oplus e_{t_{F_{2}}} \oplus R_{2} \bigoplus_{j=0}^{k_{Z_{2}}-1} \mathcal{S}_{j} .
\end{aligned}
$$

For $j=k_{Z_{2}}-1$,

$$
\begin{aligned}
& \left.\mathcal{S}_{k_{Z_{2}}-1}(f)=\operatorname{Ad}_{U\left(t_{a_{1}}-E_{2}+\left(k_{Z_{2}}-1\right) a_{1}+1\right.}, \ldots, t_{2 a_{1}-E_{2}+\left(k_{Z_{2}}-1\right) \alpha_{1}+1}\right) \\
& \left(e_{t_{a_{1}}-E_{2}+\left(k_{Z_{2}}-1\right) a_{1}+1} \oplus \cdots \oplus e_{t_{2 a_{1}-E_{2}+\left(k_{Z_{2}}-1\right) a_{1}+1}} \bigoplus_{1}^{a_{1}+1} 0\right)(f) \\
& =\operatorname{Ad}_{U\left(t_{l}-E_{\mathrm{L}}, \ldots, t_{l-\mathrm{L}}, t_{\mathrm{l}}, \ldots, t_{l+\alpha_{1}-E_{1}}\right)} \\
& \left(e_{t_{l}-E_{1}} \oplus \cdots \oplus e_{t_{l-2}} \oplus \cdots \oplus e_{t_{l}} \oplus \cdots \oplus e_{t_{l+a_{1}-E_{1}}} \bigoplus_{1}^{a_{1}+1} 0\right)(f) \\
& \sim_{2 \varepsilon} \operatorname{Ad}_{U(\underbrace{1, \ldots, 1}_{E_{1}}, s_{1}, \ldots, s_{a_{1}+1-E_{1}})}\left(\bigoplus_{1}^{E_{1}} e_{1} \oplus e_{s_{1}}\right. \\
& \left.\oplus \cdots \oplus e_{s_{a_{1}}-E_{1}} \bigoplus_{1}^{a_{1}+1}\right)(f) \\
& =\bigoplus_{1}^{E_{1}} e_{0} \oplus \operatorname{Ad}_{U\left(s_{1}, \ldots, s_{a_{1}+1-E_{1}} ; E_{1}\right)}\left(e_{s_{1}} \oplus \cdots \oplus\right. \\
& \left.e_{s_{a_{1}}-E_{1}} \bigoplus_{1}^{E_{1}} e_{\infty} \bigoplus_{1}^{a_{1}+1-E_{1}} 0\right)(f) \\
& =\bigoplus_{1}^{E_{1}} e_{0} \oplus R_{1}(f) \text {. }
\end{aligned}
$$

Then

$$
\iota_{a_{1}}^{k z_{2}}\left(L_{2}\right)(f)
$$




$$
\begin{aligned}
& \sim_{2 \varepsilon} \quad e_{t_{l-E_{1}+a_{1}}} \oplus \cdots \oplus e_{t_{F_{2}}} \bigoplus_{1}^{a_{1}+1-E_{2}} e_{0} \bigoplus_{j=0}^{k_{z_{2}}-2}\left(\bigoplus_{1}^{a_{1}+1} e_{0}\right) \bigoplus_{1}^{E_{1}} e_{0} \oplus R_{1}(f) \\
& =e_{t_{l_{-} E_{1}+a_{1}}} \oplus \cdots \oplus e_{t_{F_{2}}} \bigoplus_{1}^{k_{Z_{2}}\left(a_{1}+1\right)-E_{2}+E_{1}} e_{o} \oplus R_{1}(f) \\
& \sim_{\varepsilon} \quad e_{s_{a_{1}+1-E_{1}}} \oplus \cdots \oplus e_{s_{F_{1}-z}} \bigoplus_{1}^{z} e_{0} \oplus R_{1}(f) \\
& \sim_{\varepsilon} \quad e_{s_{a_{1}+1-E_{1}}} \oplus \cdots \oplus e_{s_{F_{1}-z}} \oplus e_{s_{F_{1}-Z+1}} \oplus \cdots \oplus e_{s_{F_{1}}} \oplus R_{1}(f) \\
& =\quad L_{1}(f)=f_{s} \phi^{\prime}(f) .
\end{aligned}
$$

Therefore, for $f \in F$,

$$
\left\|f_{s} \psi^{\prime}(f)-f_{s} \phi^{\prime}(f)\right\|<4 \varepsilon
$$

Subcase 3.2: $k_{2}=k_{Z_{2}}-1$.

Using the estimates for $R_{2}$ and $\mathcal{S}_{j}$ given in equations (4.24) and (4.25) respectively,

$$
\begin{aligned}
& \iota_{a_{1}}^{c_{z_{2}-1}}\left(L_{2}\right)(f) \\
& =e_{t_{a_{1}-E_{2}+\left(k z_{2}-1\right) a_{1}+1}} \oplus \cdots \oplus e_{t_{F_{2}}} \oplus R_{2} \bigoplus_{j=0}^{k_{Z_{2}}-2} \mathcal{S}_{j}(f) \\
& =e_{t_{l-E_{1}}} \oplus \cdots \oplus e_{t_{F_{2}}} \oplus R_{2} \bigoplus_{j=0}^{k_{z_{2}-2}} \mathcal{S}_{j}(f) \\
& \sim e_{t_{l-E_{1}}} \oplus \cdots \oplus e_{t_{F_{2}}} \bigoplus_{1}^{z-a_{1}-E_{1}} e_{0} \\
& =e_{t_{F_{2}-r_{2}}} \oplus \cdots \oplus e_{t_{F_{2}}} \bigoplus_{1}^{Z-a_{1}-E_{1}} e_{0}
\end{aligned}
$$

The last line follows from the line above it using the following calculation:

$$
F_{2}-\left(l-E_{1}\right)=F_{2}+E_{2}-a k_{Z_{2}}-1
$$




$$
\begin{aligned}
& =F_{2}+E_{2}-a_{1}\left(k_{2}+1\right)-1 \\
& =F_{2}-\left(a_{1}-E_{2}\right)-a_{1} k_{2}-1 \\
& =r_{2}-1 .
\end{aligned}
$$

Therefore,

$$
\begin{aligned}
& f_{s} \psi^{\prime}(f)=\iota_{\tau_{2}} l_{a_{1}}^{k} k_{Z_{2}-1}\left(L_{2}\right)(f) \\
& z-a_{1}-E_{1}-\left(a_{1}-r_{2}\right) \\
& \sim_{2 \varepsilon} \quad \bigoplus_{1} \quad e_{0} \oplus \operatorname{Ad}_{U\left\langle t_{l-E_{1}}, \ldots, t_{F_{2}},\right.}, \underbrace{0, \ldots, 0)}_{a_{1}-r_{2}}\left(e_{t_{t-E_{1}}}\right. \\
& \left.\oplus \cdots \oplus e_{t_{F_{2}}} \bigoplus_{1}^{a_{1}-r_{2}} e_{0} \bigoplus_{1}^{a_{1}+1} 0\right)(f) \\
& Z-2 a_{1}-E_{1}+r_{2} \\
& \sim_{2 \varepsilon} \quad \bigoplus_{1} \quad e_{0} \oplus \operatorname{Ad}_{U}(\underbrace{\left.1, \ldots, 1, t_{t}, \ldots, t_{F_{2}}, 0, \ldots, 0\right)}_{E_{1}} \\
& \left(\bigoplus_{1}^{E_{1}} e_{1} \oplus e_{t_{l}} \oplus \cdots \oplus e_{t_{F_{2}}} \bigoplus_{1}^{a_{1}-r_{2}} e_{0} \bigoplus_{1}^{a_{1}+1} 0\right)(f) \\
& \sim_{2 \varepsilon} \bigoplus_{1}^{Z-2 a_{1}-E_{1}+r_{2}} e_{0} \bigoplus_{1}^{E_{1}} e_{0} \oplus \operatorname{Ad}_{U\left(s_{1}, \ldots, s_{a_{1}}-E_{1} ; E_{1}\right)}\left(e_{s_{1}}\right. \\
& \left.\oplus \cdots \oplus e_{s_{a_{1}-E_{1}}} \bigoplus_{1}^{E_{1}} e_{\infty} \bigoplus_{1}^{a_{1}+1-E_{1}}\right)(f) \\
& =\bigoplus_{1}^{z-2 a_{1}+r_{2}} e_{0} \oplus R_{1}(f) .
\end{aligned}
$$

The second last line follows from the line above it due to the following calculation and the relations $\left|t_{l-1+i}-s_{i}\right|<\delta$ and $\left|s_{i}-0\right|<\delta$ in equation (4.17),

$$
\begin{aligned}
\left(F_{2}-l\right)+1+\left(a_{1}-r_{2}\right) & =F_{2}-r_{2}-l+1+a_{1} \\
& =\left(a_{1}-E_{2}\right)+a_{1} k_{2}-a_{1}\left(k_{2}+1\right)-E_{1}+E_{2}+a_{1}
\end{aligned}
$$




$$
=\left(a_{1}-E_{1}\right)
$$

Notice that by the following calculation $Z-2 a_{1}+r_{2}=F_{1}-\left(a_{1}-E_{1}\right)$ :

$$
\begin{aligned}
Z-2 a_{1}+r_{2} & =\left(a_{1}+1\right)\left(k_{Z_{2}}\right)+E_{1}-E_{2}-a_{1}+r_{2} \\
& =a_{1} k_{Z_{2}}+E_{1}-E_{2}+k_{Z_{2}}-a_{1}+r_{2} \\
& =a_{1}\left(k_{2}+1\right)-E_{2}+r_{2}+k_{Z_{2}}-\left(a_{1}-E_{1}\right) \\
& =\left(a_{1}-E_{2}\right)+a_{1} k_{2}+r_{2}+k_{Z_{2}}-\left(a_{1}-E_{1}\right) \\
& =F_{2}+k_{Z_{2}}-\left(a_{1}-E_{1}\right) \\
& =F_{1}-\left(a_{1}-E_{1}\right) .
\end{aligned}
$$

By equation (4.17), $\left|s_{i}-0\right|<\delta$ for $i=F_{1}, \ldots, F_{1}-Z+1$. We can find an equivalent expression for $F_{1}-Z+1$ which will be helpful for the calculation that follows;

$$
\begin{aligned}
F_{1}-Z+1 & =F_{2}-l+2 \\
& =F_{2}-\left(a_{1} k_{f}+E_{1}-E_{2}\right)+1 \\
& =F_{2}-a_{1}\left(k_{2}+1\right)-E_{2}+E_{2}+1 \\
& =F_{2}-\left(a_{1}-E_{2}\right)-a_{1} k_{2}-E_{1}+1 \\
& =r_{2}-E_{1}+1 .
\end{aligned}
$$

As $r_{2}-E_{1}+1 \leq a_{1}-E_{1}+1,\left|s_{i}-0\right|<\delta$ for $i \geq a_{1}-E_{1}+1$, and therefore:

$$
\begin{aligned}
\bigoplus_{1}^{Z-2 a_{1}+r_{2}} e_{0} \oplus R_{1}(f) & =\bigoplus_{1}^{F_{1}-\left(a_{1}-E_{1}\right)} e_{0} \oplus R_{1}(f) \\
& \sim_{\varepsilon} e_{s_{a_{1}-E_{1}+1}} \oplus \cdots \oplus e_{s F_{1}} \oplus R_{1}(f) \\
& =f_{s} \phi^{\prime}(f) .
\end{aligned}
$$


It follows that for all $f \in F$,

$$
\left\|f_{s} \psi^{\prime}(f)-f_{s} \phi^{\prime}(f)\right\|<7 \varepsilon
$$

By Proposition 4.2.2, Subcases 3.1 and 3.2 are exhaustive for Subcase 3. By the estimates made in the two subcases, for all $f \in F$,

$$
\left\|f_{s} \psi^{\prime}(f)-f_{s} \phi^{\prime}(f)\right\|<7 \varepsilon
$$

Case 4: $Z_{1} \geq a_{1}+1-E_{1}$ and $Z_{2} \geq a_{1}+1-E_{2}$.

Without loss of generality it is possible to assume that $Z_{2}+E_{2} \geq Z_{1}+E_{1}$, and if equality holds in the preceding inequality then $E_{1} \geq E_{2}$. From the identity $\operatorname{dim}\left(D_{2}\right)=\left(a_{1}+1\right) F_{1}+Z_{1}+E_{1}=\left(a_{1}+1\right) F_{2}+Z_{2}+E_{2}$ it is clear that $Z_{2}+E_{2}-\left(Z_{1}+E_{1}\right)=Z_{2}-\left(a_{1}+1-E_{2}\right)-\left(Z_{1}-\left(a_{1}+1-E_{1}\right)\right)$ is divisible by $a_{1}+1$. Let

$$
Z_{2}-\left(a_{1}+1-E_{2}\right)-\left(Z_{1}-\left(a_{1}+1-E_{1}\right)\right)=k_{f}\left(a_{1}+1\right)
$$

Then

$$
F_{1}=F_{2}+k_{f}
$$

As $Z_{1}-\left(a_{1}+1-E_{1}\right)=k_{Z_{1}}\left(a_{1}+1\right)+r_{Z_{1}}$ and $Z_{2}-\left(a_{1}+1-E_{2}\right)=k_{Z_{2}}\left(a_{1}+1\right)+r_{Z_{2}}$;

$$
k_{Z_{2}}=k_{Z_{1}}+k_{f}
$$

and

$$
r_{Z_{2}}=r_{Z_{1}}
$$

We have assumed that $Z_{2}+E_{2} \geq Z_{2}+E_{1}$ and if equality holds then $E_{1} \geq E_{2}$. As $Z_{2}+E_{2}-\left(Z_{1}+E_{1}\right)=\left(k_{Z_{2}}-k_{Z_{1}}\right)\left(a_{1}+1\right)+r_{Z_{2}}-r_{Z_{1}}=\left(k_{Z_{2}}-k_{Z_{1}}\right)\left(a_{1}+1\right)$, 
another way to rephrase our assumption is that if $k_{Z_{2}}=k_{Z_{1}}$ then $E_{1} \geq E_{2}$. The difference in the number of eigenvalues of $\phi^{\prime}(h)(s)$ with eigenvalue equal to one and the number of eigenvalues of $\psi^{\prime}(h)(s)$ with eigenvalue equal to one is $a_{1} F_{1}+E_{1}-\left(a_{1} F_{2}+E_{2}\right)=a_{1} k_{f}+E_{1}-E_{2}$. Let

$$
l-1=a_{1} k_{f}+E_{1}-E_{2} .
$$

As in Cases 2 and 3, comparing the eigenvalues of $\phi^{\prime}(h)(s)$ and $\psi^{\prime}(h)(s)$ it is possible to derive the set of equations (4.17) with the value of $l$ given above. The following proposition will be important for the extensive subcase analysis that is necessary for this case of the proof.

Proposition 4.2.3 Assume that $Z_{2}+E_{2} \geq Z_{1}+E_{1}$ and $E_{1} \geq E_{2}$ if $Z_{2}+E_{2}=$ $Z_{1}+E_{1}$. Then $k_{1} \geq k_{2}-1$ and $k_{1} \geq k_{2}$ if $k_{Z_{2}}=k_{Z_{1}}$.

Proof. The proof is given by a simple counting argument; as $\operatorname{dim}\left(D_{2}\right)=$ $\left(a_{1}+1\right) F_{i}+Z_{i}+E_{i}$ for $i=1,2$,

$$
\begin{aligned}
& \left(a_{1}+1\right)\left(\left(a_{1}-E_{1}\right)+a_{1} k_{1}+r_{1}\right)+\left(a_{1}+1-E_{1}\right)+k_{Z_{1}}\left(a_{1}+1\right)+r_{Z_{1}}+E_{1} \\
& =\left(a_{1}+1\right)\left(\left(a_{1}-E_{2}\right)+a_{1} k_{2}+r_{2}\right)+\left(a_{1}+1-E_{2}\right)+k_{Z_{2}}\left(a_{1}+1\right)+r_{Z_{2}}+E_{2} .
\end{aligned}
$$

We can simplify the above expression to:

$$
a_{1}\left(k_{1}-k_{2}\right)=\left(k_{Z_{2}}-k_{Z_{1}}\right)+\left(E_{1}-E_{2}\right)+\left(r_{2}-r_{2}\right) .
$$

As $k_{Z_{2}} \geq k_{Z_{1}}$, the right-hand side of the above expression is bounded below (strictly) by $-2 a_{1}$. Therefore, $k_{1} \geq k_{2}-1$. If $k_{Z_{2}}=k_{Z_{1}}$ then $E_{1} \geq E_{2}$ and the right-hand side will be strictly bounded below by $-a_{1}$; it follows that $k_{1} \geq k_{2}$ in this case. 
The proof of the following proposition is similar to that given for Proposition 4.2.2.

Proposition 4.2.4 $k_{2} \geq k_{f}-1$.

Proof. The argument is as follows: at $s, \phi^{\prime}(h)$ has $a_{1}\left(F_{1}-F_{2}\right)+E_{1}-E_{2}=$ $a_{1} k_{f}+E_{1}-E_{2}$ more eigenvalues equal to one than $\psi^{\prime}(h)$. Let $s_{i}$ and $t_{i}$ denote the eigenvalues of $\phi^{\prime}(h)$ and $\psi^{\prime}(h)$ that are not equal to zero or one. Then $\left|t_{i}-1\right|<\delta$ for at least $a_{1} k_{f}+E_{1}-E_{2}$ values of $i$, and hence $F_{2} \geq a_{1} k_{f}+E_{1}-E_{2}$. Then $a_{1} k_{2}+r_{2}=F_{2}-\left(a_{1}-E_{2}\right) \geq a_{1}\left(k_{f}-1\right)+E_{1}$, and hence

$$
a\left(k_{2}-k_{f}+1\right) \geq E_{1}-r_{2}>-a_{1}
$$

It follows from the above inequality that $k_{2} \geq k_{f}-1$.

Subcase 4.1: $k_{Z_{2}} \leq k_{2}$ and if $k_{Z_{2}}=k_{2}$ then $r_{Z_{2}} \leq r_{2}$.

By equation (4.28), and the assumption that $k_{Z_{2}} \leq k_{2}$,

$$
k_{f} \leq k_{2}
$$

Therefore, by the construction for standard maps given in Section 4.1, Subcase 3.1 ,

$$
\begin{aligned}
& f_{s} \psi^{\prime}=\iota_{r Z_{2}, r_{Z_{2}}}^{\prime}{ }^{\iota_{a_{1}}^{k} Z_{Z_{2}}} L_{2} \\
& =\iota_{r Z_{2}, r Z_{2}}^{r} \iota_{a_{1}}^{k Z_{1}} \iota_{a_{1}}^{k_{f}} L_{2} \\
& =\iota_{r Z_{2}, r z_{2}}^{\prime} \iota_{a_{1}}^{k_{Z_{1}}}\left(e_{t_{l-E_{1}+a_{1}}} \oplus \cdots \oplus e_{t_{F_{2}}} \oplus R_{2} \bigoplus_{j=0}^{k_{f}-1} \mathcal{S}_{j}\right)
\end{aligned}
$$

and

$$
f_{s} \phi^{\prime}=\iota_{r z_{2}, r z_{2}}^{\prime} \iota_{a_{1}}^{k_{z_{1}}} L_{1}
$$


Both $\iota_{\alpha_{1}}^{k_{f}} L_{2}$ and $L_{1}$ are representations of $A_{1}$ in standard form. In fact from the definition of $L_{1}$ given in Section 4.1, Subcase 3.1, it follows that

$$
L_{1}=\operatorname{Std}\left(t_{1}, \ldots, t_{F_{1}}, E_{1}, Z_{1}^{\prime}\right)
$$

with $Z_{1}^{\prime}=a_{1}+1-E_{1}$. Furthermore, from the assumption that $Z_{2}+E_{2} \geq$ $Z_{1}+E_{1}$, it follows that

$$
\iota_{a_{1}}^{k_{f}} L_{2}=\operatorname{Std}\left(s_{2}, \ldots, s_{F_{2}}, E_{2}, Z_{2}^{\prime}\right)
$$

with $Z_{2}^{\prime}=k_{f}\left(a_{1}+1\right)+a_{1}+1-E_{1}$. Therefore, by Case 3

$$
L_{1}(f) \sim \sim_{6 \varepsilon} \iota_{a_{1}}^{k_{f}} L_{2}(f)
$$

for all $f \in F$.

From the argument given above it suffices to show that the maps $\iota_{a_{1}}$ and $\iota_{r Z_{2}, r Z_{2}}^{\prime}$ act approximately the same on $L_{1}$ and $\iota_{a_{1}}^{k_{f}} L_{2}$. The maps act by powers of the unitary shift $V$, and a unitary twist $U(\bullet, \ldots, \bullet)$ :

$$
\begin{aligned}
\iota_{a_{1}}\left(\iota_{a_{1}}^{p+k_{f}} L_{2}\right)= & \operatorname{Ad}_{U\left(t_{t-E_{1}+(p+1) a_{1}}, \ldots, t_{l-E_{1}+(p+2) a_{1}-1}\right)}\left(\operatorname{Ad}_{\left(V^{*}\right)^{a_{1}}}\left(\iota_{a_{1}}^{p+k_{f}} L_{2}\right) \oplus_{1}^{a_{1}+1} 0\right) \\
\iota_{a_{1}}\left(\iota_{a_{1}}^{p} L_{1}\right)= & \operatorname{Ad}_{U\left(s_{1-E_{1}+(p+1) a_{1}}, \ldots, s_{1-E_{1}+(p+2) a_{1}-1}\right)}( \\
& \left.\operatorname{Ad}_{\left(V^{*}\right)^{a_{1}}}\left(\iota_{a_{1}}^{p} L_{1}\right) \oplus_{1}^{a_{1}+1} 0\right)
\end{aligned}
$$

for $p \leq k_{Z_{1}}-1$, and

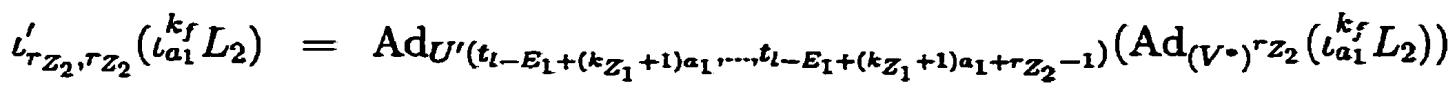

$$
\begin{aligned}
& \iota_{\tau Z_{2}, r Z_{2}}^{\prime}\left(L_{1}\right)=\operatorname{Ad}_{U^{\prime}\left(s_{1}-E_{1}+\left(k_{Z_{1}}+1\right) a_{1}, \ldots, s_{1}-E_{1}+\left(k_{Z_{1}}+1\right) a_{1}+r Z_{2}-1\right.}\left(\operatorname{Ad}_{\left(V^{*}\right)^{r Z_{2}}}\left(L_{1}\right)\right) .
\end{aligned}
$$


As $\left|t_{l-1+i}-s_{i}\right|<\delta$ (as noted in (4.17)),

$$
\begin{aligned}
& \| U\left(t_{l-E_{1}+(p+1) a_{1}}, \ldots, t_{l-E_{1}+(p+2) a_{1}-1}\right) \\
& \quad-U\left(s_{1-E_{1}+(p+1) a_{1}}, \ldots, s_{1-E_{1}+(p+2) a_{1}-1}\right) \|<\varepsilon / 2
\end{aligned}
$$

and

$$
\begin{aligned}
& \| U^{\prime}\left(t_{l-E_{1}+\left(k z_{1}+1\right) a_{1}}, \ldots, t_{l-E_{1}+\left(k_{Z_{1}}+1\right) a_{1}+r z_{2}-1}\right) \\
& \quad-U^{\prime}\left(s_{1-E_{1}+\left(k_{Z_{1}}+1\right) a_{1}}, \ldots, s_{1-E_{1}+\left(k_{Z_{1}}+1\right) a_{1}+r Z_{2}-1}\right) \|<\varepsilon / 2
\end{aligned}
$$

For $p \leq k_{Z_{1}}-1$, let $U_{p}$ denote the unitary twist given by the action of $\iota_{a_{1}}$ on $\iota_{a_{1}}^{p} L_{1}$ or $\iota_{a_{1}}^{p+k_{f}} L_{2}$, then because of the factor of a unitary shift $V$ in the definition of the standard maps, $U_{p}$ acts as the identity except on the $\left(a_{1}+1\right)^{2}$ dimensional subspace

$$
\mathcal{H}_{p}=\operatorname{sp}\left\{\xi_{i}\right\}_{i=m-k_{Z_{1}}\left(a_{1}\right)\left(a_{1}+1\right)-r Z_{1}\left(a_{1}+1\right)-r Z_{1}\left(a_{1}+1\right)+(p-1)\left(a_{1}+1\right)^{2}}^{m}
$$

where $m$ is the dimension of the Hilbert space on which $f_{s} \psi^{\prime}$ or $f_{s} \phi^{\prime}$ is represented. For $p \neq q, \mathcal{H}_{p} \perp \mathcal{H}_{q}$, hence from equations (4.30) and (4.31),

$$
\left\|\iota_{a_{1}}^{p+1+k_{f}} L_{2}(f)-\iota_{a_{1}}^{p+1} L_{1}(f)\right\|<7 \varepsilon
$$

for $0 \leq p \leq k_{Z_{1}}-1$. In particular for $p=k_{Z_{1}}$,

$$
\left\|\iota_{a_{1}}^{k_{Z_{1}}+k_{f}} L_{2}(f)-\iota_{a_{1}}^{k_{Z_{1}}} L_{1}(f)\right\|<7 \varepsilon
$$

It should be noted that to prove the above estimate the only hypothesis that was used is that $k_{Z_{2}} \leq k_{2}$. Equation (4.33) will be used repeatedly in the remaining subcases. As the unitaries implementing the action of $\iota_{r Z_{2}, r Z_{2}}^{\prime}$ on 


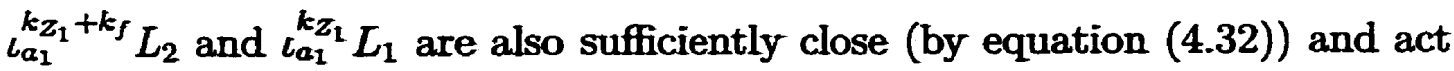
on a subspace orthogonal to $\mathcal{H}_{q}$ for $0 \leq q \leq k_{Z_{1}}-1$,

$$
\left\|\iota_{r Z_{2}, r Z_{2}}^{\prime} \iota_{a_{1}}^{k_{Z_{1}}+k_{f}} L_{2}(f)-\iota_{r Z_{2}, r Z_{2}}^{\prime}{ }^{c_{a_{1}}} L_{1}(f)\right\|<7 \varepsilon
$$

for all $f \in F$. This completes the proof of Subcase 4.1 .

The following proposition and corollary will be used in Subcases 4.2 and 4.3:

Proposition 4.2.5 If $k_{Z_{1}}=k_{1}$ and $k_{Z_{2}}=k_{2}$ (or if $k_{Z_{1}}=k_{1}+1$ and $k_{Z_{2}}=$ $\left.k_{2}+1\right)$ then

$$
k_{Z_{2}}=k_{Z_{1}}, k_{1}=k_{2} \text { and } E_{1}-E_{2}=r_{1}-r_{2}
$$

or

$$
k_{Z_{2}}=k_{Z_{1}}+1, k_{1}=k_{2}-1 \text { and } E_{1}-E_{2}=r_{1}-r_{2}+a_{1}+1
$$

Proof. Recall that

$\left(a_{1}+1\right) F_{1}+\left(a_{1}+1\right)+\left(Z_{1}-\left(a_{1}+1-E_{1}\right)\right)=\left(a_{1}+1\right) F_{2}+\left(a_{1}+1\right)+\left(Z_{2}-\left(a_{1}+1-E_{2}\right)\right)$

and hence $\left(k_{Z_{1}}-k_{Z_{2}}\right)\left(a_{1}+1\right)=\left(a_{1}+1\right)\left(F_{2}-F_{1}\right)$, or $k_{Z_{1}}-k_{Z_{2}}=E_{1}-E_{2}+$ $r_{2}-r_{1}+\left(k_{2}-k_{1}\right) a_{1}$. Substituting $k_{1}=k_{Z_{1}}$ and $k_{2}=k_{Z_{2}}$ (or $k_{Z_{1}}=k_{1}+1$ and $k_{Z_{2}}=k_{2}+1$ ),

$$
\begin{aligned}
\left(k_{Z_{1}}-k_{Z_{2}}\right)\left(a_{1}+1\right) & =E_{1}-E_{2}+r_{2}-r_{1} \\
\left(k_{1}-k_{2}\right)\left(a_{1}+1\right) & =E_{1}-E_{2}+r_{2}-r_{1} .
\end{aligned}
$$

As $E_{i}, r_{i}<a_{1}, E_{1}-E_{2}+r_{2}-r_{1}>-2 a_{1}$. By equation (4.28), $k_{Z_{2}} \geq k_{Z_{1}}$. Using the lower bound for $E_{1}-E_{2}+r_{2}-r_{1}$ and equation (4.34) we can 
conclude that either

$$
k_{Z_{2}}=k_{Z_{1}} \text { or } k_{Z_{2}}=k_{Z_{1}}+1 \text {. }
$$

In the former case, by equation (4.35),

$$
k_{1}=k_{2} \text { and } E_{1}-E_{2}=r_{1}-r_{2} .
$$

In the latter case,

$$
k_{1}=k_{2}-1 \text { and } E_{1}-E_{2}=r_{1}-r_{2}+a_{1}+1
$$

Corollary 4.2.1 With the same assumptions as in Proposition 4.2.5, $Z=$ $r_{1}-r_{2}$ and $l=r_{1}-r_{2}$.

\section{Proof.}

$$
\begin{aligned}
Z & =Z_{2}-\left(a_{1}+1-E_{2}\right)-\left(Z_{1}-\left(a_{1}+1-E_{1}\right)\right)+E_{1}-E_{2} \\
& =\left(k_{Z_{2}}-k_{Z_{2}}\right)\left(a_{1}+1\right)+E_{1}-E_{2} .
\end{aligned}
$$

As $k_{Z_{2}}=k_{Z_{1}}$ and $E_{1}-E_{2}=r_{1}-r_{2}$, or $k_{Z_{2}}=k_{Z_{1}}+1$ and $E_{1}-E_{2}=$ $r_{1}-r_{2}+a_{1}+1$ the result follows.

Subcase 4.2: $k_{Z_{2}}=k_{2}$ and $r_{Z_{2}}>r_{2}$.

There are three subcases:

Subcase 4.2.1: $k_{Z_{1}}=k_{1}$ and $r_{Z_{2}}>r_{1}$.

From the standard map construction, as given in Section 4.1, Subcase 3.3,

$$
f_{s} \phi^{\prime}=\iota_{\tau_{1}, r z_{2}}^{\prime} l_{a_{1}}^{k z_{z_{1}}} L_{1}
$$




$$
f_{s} \psi^{\prime}=\iota_{\tau_{2}, Z_{Z_{2}}}^{\prime} \iota_{a_{I}}^{k_{Z_{1}+k_{f}}} L_{2}
$$

As $k_{Z_{2}} \leq k_{2}$, by equation (4.33), \|\|$_{a_{1}}^{k_{Z_{1}}+k_{f}} L_{2}(f)-\iota_{a_{1}}^{k_{Z_{1}}} L_{1}(f) \|<7 \varepsilon$, and therefore it suffices to prove that the unitaries implementing the action of $\iota_{r_{1}, r z_{2}}^{\prime}$ and $\iota_{r_{2}, r z_{2}}^{\prime}$, namely,

$$
U^{\prime}(\underbrace{s_{F_{1}-r_{1}+1, \ldots, s_{F_{1}}, 0, \ldots, 0}}_{r z_{2}})
$$

and

$$
U^{\prime}(\underbrace{t_{F_{2}-r_{2}+1}, \ldots, t_{F_{2}}, 0, \ldots, 0}_{r z_{2}})
$$

on $\iota_{a_{1}}^{k_{Z_{1}}} L_{1}$ and $\iota_{a_{1}}^{\boldsymbol{k}_{Z_{1}}+k_{f}} L_{2}$ respectively, are sufficiently close.

In Corollary 4.2.1 we found that $Z=l=r_{1}-r_{2}$. Therefore, by equation (4.17), $\left|s_{i}-0\right|<\delta$ for $i=F_{1}, F_{1}-1, \ldots, F_{1}-\left(r_{1}-r_{2}\right)+1$ and $\left|t_{r_{1}-r_{2}+i}-s_{i}\right|<\delta$ for $i=1,2, \ldots, F_{1}-\left(r_{1}-r_{2}\right)$. Then

$$
\begin{aligned}
& U^{\prime}\left(s_{F_{1}-r_{1}+1}, \ldots, s_{F_{1}}, 0, \ldots, 0\right) \\
& \quad=U^{\prime}\left(s_{F_{1}-r_{1}+1}, \ldots, s_{F_{1}-\left(r_{1}-r_{2}\right)}, s_{F_{1}-\left(r_{1}-r_{2}\right)+1}, \ldots, s_{F_{1}}, 0, \ldots, 0\right) \\
& \sim_{\varepsilon / 2} U^{\prime}\left(t_{F_{2}-r_{2}+1}, \ldots, t_{F_{2}}, 0, \ldots, 0\right) .
\end{aligned}
$$

Subcase 4.2.2: $k_{Z_{1}} \leq k_{1}$ and if $k_{1}=k_{Z_{1}}$ then $r_{Z_{2}} \leq r_{1}$.

The unitary implementing the action of $\iota_{r_{Z_{2}}, r Z_{2}}^{\prime}$ on $\iota_{a_{1}}^{k_{Z_{1}}} L_{1}$ is

$$
U^{\prime}\left(s_{1-E_{1}+\left(k_{Z_{1}}+1\right) a_{1}}, \ldots, s_{\left.1-E_{1}+\left(k_{z_{1}}+1\right) a_{1}+r z_{2}-1\right)}\right. \text {. }
$$

We can use equation (4.17) for comparing eigenvalues and equation (4.31) for the computing the indices of the unitary:

$$
U^{\prime}\left(s_{1-E_{1}+\left(k_{Z_{1}}+1\right) a_{1}}, \ldots, s_{1-E_{1}+\left(k_{Z_{1}}+1\right) a_{1}+r_{Z_{2}}-1}\right)
$$




$$
\begin{aligned}
&= U^{\prime}\left(s_{1-E_{1}+\left(k_{Z_{1}}+1\right) a_{1}}, \ldots, s_{1-E_{1}+\left(k_{Z_{1}}+1\right) a_{1}+r_{1}-1}\right. \\
&\left.s_{1-E_{1}+\left(k_{Z_{1}}+1\right) a_{1}+r_{1}}, \ldots, s_{1-E_{1}+\left(k_{Z_{1}}+1\right) a_{1}+r_{Z_{2}}-1}\right) \\
& \sim_{E / 2} \quad U^{\prime}\left(t_{l-E_{1}+\left(k_{Z_{1}}+1\right) a_{1}}, \ldots, t_{l-E_{1}+\left(k_{Z_{1}}+1\right) a_{1}+r_{1}-1}, 0, \ldots, 0\right) \\
&=\quad U^{\prime}\left(t_{F_{2}-r_{2}+1}, \ldots, t_{F_{2}}, 0, \ldots, 0\right) .
\end{aligned}
$$

(The last line follows from the line above it using the fact that $k_{Z_{1}}+k_{f}=k_{Z_{2}}$ and $k_{Z_{2}}=k_{2}$.) As $U^{\prime}\left(t_{F_{2}-r_{2}+1}, \ldots, t_{F_{2}}, 0, \ldots, 0\right)$ is the unitary implementing the action of $\iota_{\tau 2, r z_{2}}^{\prime}$ on $\iota_{a_{1}}^{k_{Z_{2}}+k_{f}} L_{2}$, and $\iota_{\alpha_{1}}^{k z_{1}}\left(L_{1}\right)(f) \sim_{7 \varepsilon} \iota_{a_{1}}^{k_{Z_{1}}+k_{f}} L_{2}(f)$ by equation (4.33), it follows that

$$
\begin{aligned}
& f_{s} \phi^{\prime}(f)=\iota_{r Z_{2}, r Z_{2}}^{\prime}{ }^{\iota_{a_{1}}^{k} z_{1}} L_{1}(f) \\
& \sim_{7 \varepsilon} \quad \iota_{r_{2}, r z_{2}}^{\prime} \iota_{\alpha_{1}}^{k_{Z_{1}}+k_{f}} L_{2}(f) \\
& =f_{s} \psi^{\prime}(f) \text {. }
\end{aligned}
$$

We will show that the following subcase never occurs.

Subcase 4.2.3: $k_{1}=k_{Z_{1}}-1$.

Under the assumption $k_{2}=k_{Z_{2}}$, by Proposition 4.2.3 and equation (4.28),

$$
k_{1} \geq k_{2}-1=k_{Z_{2}}-1 \geq k_{Z_{1}}-1
$$

Under our assumption $k_{1}=k_{Z_{1}}-1$ and therefore we must have equality everywhere in the above inequality:

$$
k_{1}=k_{2}-1=k_{Z_{2}}-1=k_{Z_{1}}-1
$$

By assumption, if $k_{Z_{2}}=k_{Z_{1}}$ then $E_{1}-E_{2}>0$. By Proposition 4.2.3, $k_{1} \geq k_{2}$, contradicting equation (4.37). 
To complete Subcase 4.2 we need to argue that Subcases 4.2.1,4.2.2 and 4.2.3 are exhaustive. By equation (4.36), $k_{1} \geq k_{Z_{1}}-1$. It follows that the three subcases are exhaustive for subcase 4.2. Therefore, for $f \in F$,

$$
\left\|f_{s} \phi^{\prime}(f)-f_{s} \psi^{\prime}(f)\right\|<7 \varepsilon
$$

Subcase 4.3: $k_{Z_{2}}=k_{2}+1$ and $r_{Z_{2}}=0$.

There are three subcases depending on the value of $k_{Z_{1}}$.

Subcase 4.3.1: $k_{Z_{1}} \leq k_{1}$.

By the construction given in Subcases 3.1 and 3.3 of Section 4.1,

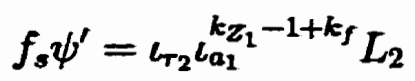

and

$$
f_{s} \phi^{\prime}=\iota_{a_{1}}^{k_{Z_{1}}} L_{1}
$$

respectively. As $k_{Z_{1}}-1+k_{f}=k_{Z_{2}}-1 \leq k_{2}$, by equation(4.33),

$$
\left\|\iota_{a_{1}}^{k_{Z_{1}}-1+k_{f}} L_{2}(f)-\iota_{a_{1}}^{k_{Z_{1}}-1} L_{1}(f)\right\|<7 \varepsilon
$$

Therefore it suffices to shown that the unitary implementing the action of $\iota_{r_{2}}$ on $\iota_{a_{1}}^{k_{Z_{1}}-1+k_{f}} L_{2}$ is close to the unitary implementing the action of $\iota_{a_{1}}$ on $\iota_{a_{1}}^{k_{Z_{1}}-1} L_{1}$. The unitary implementing the action of $\iota_{a_{1}}$ on $\iota_{a_{1}}^{k_{Z_{1}}-1} L_{1}$ is

$$
\begin{aligned}
& U\left(s_{1-E_{1}+k_{Z_{1}} a_{1}}, \ldots, s_{1-E_{1}+k_{Z_{1}+a_{1}} a_{1}}\right) \\
& \quad=U\left(s_{1-E_{1}+k_{Z_{1}} a_{1}}, \ldots, s_{1-E_{1}+k_{Z_{1}} a_{1}+r_{2}-1}, s_{1-E_{1}+k_{Z_{1}} a_{1}+r_{2}}, \ldots, s_{1-E_{1}+k_{Z_{1}+1} a_{1}-1}\right) \\
& \sim_{\varepsilon / 2} U\left(t_{l^{\prime}-E_{1}+k_{Z_{1}} a_{1}}, \ldots, t_{l^{\prime}-E_{1}+k_{Z_{1}} a_{1}+r_{2}-1}, 0, \ldots, 0\right) \\
& \quad=U\left(t_{F_{2}-r_{2}+1}, \ldots, t_{F_{2}}, 0, \ldots, 0\right) .
\end{aligned}
$$


(The last line follows from the line above it using the fact that $k_{Z_{1}}+k_{f}=k_{Z_{2}}$, $k_{Z_{2}}=k_{2}+1$ and the comparison of eigenvalues given in (4.17).) The unitary implementing the action of $\iota_{r_{2}}$ on $\iota_{a_{1}}^{k_{Z_{1}}-1+k_{f}} L_{2}$ is $U\left(t_{F_{2}-r_{2}+1}, \ldots, t_{F_{2}}, 0, \ldots, 0\right)$. Therefore, for $f \in F$,

$$
\left\|c_{\tau_{2}} \ell_{a_{1}}^{k_{Z_{1}}-1+k_{f}} L_{2}(f)-\iota_{a_{1}} \ell_{a_{1}}^{k_{Z_{1}}-1} L_{1}(f)\right\|<7 \varepsilon
$$

Subcase 4.3.2: $k_{Z_{1}}=k_{1}+1$.

The argument in this subcase is very similar to that given in Subcase 4.2.1. From the standard map construction in Section 4.1, Subcase 3.3,

$$
f_{s} \phi^{\prime}=\iota_{r_{1}} \iota_{a_{1}}^{k_{Z_{1}}-1} L_{1}
$$

By equation (4.33), as $k_{Z_{1}}-1+k_{f}=k_{Z_{2}}-1 \leq k_{2}, \| L_{a_{1}}^{k_{Z_{1}}-1+k_{f}} L_{2}(f)-$ $\iota_{a_{1}}^{k_{Z_{1}}-1} L_{1}(f) \|<7 \varepsilon$, hence it suffices to prove that the unitaries implementing the action of $\iota_{\tau_{1}}$ and $\iota_{\tau_{2}}$, namely,

$$
U(\underbrace{s_{F_{1}-r_{1}+1}, \ldots, s_{F_{1}}, 0, \ldots, 0}_{a_{1}})
$$

and

$$
U(\underbrace{t_{F_{2}-r_{2}+1}, \ldots, t_{F_{2}}, 0, \ldots, 0}_{a_{1}})
$$

on $\iota_{a_{1}}^{k_{Z_{1}}-1} L_{1}$ and $\iota_{a_{1}}^{k_{Z_{1}}-1+k_{f}} L_{2}$ respectively, are sufficiently close. By equation (4.17) and Corollary 4.2.1, $\left|s_{i}-0\right|<\delta$ for $i=F_{1}, F_{1}-1, \ldots, F_{1}-\left(r_{1}-r_{2}\right)+1$ and $\left|t_{r_{1}-r_{2}+i}-s_{i}\right|<\delta$ for $i=1,2, \ldots, F_{1}-\left(r_{1}-r_{2}\right)$. Therefore

$$
\begin{aligned}
& U\left(s_{F_{1}-r_{1}+1}, \ldots, s_{F_{1}}, 0, \ldots, 0\right) \\
& \quad=U\left(s_{F_{1}-r_{1}+1}, \ldots, s_{F_{1}-\left(r_{1}-r_{2}\right)}, s_{F_{1}-\left(r_{1}-r_{2}\right)+1}, \ldots, s_{F_{1}}, 0, \ldots, 0\right) \\
& \quad \sim_{\varepsilon / 2} U\left(t_{F_{2}-r_{2}+1}, \ldots, t_{F_{2}}, 0, \ldots, 0\right) .
\end{aligned}
$$


Therefore,

$$
\iota_{r_{1}} \iota_{a_{1}}^{k_{Z_{1}}-1} L_{1}(f) \sim \sim_{7 \varepsilon} \quad \iota_{r_{2}} l_{a_{1}}^{k_{Z_{1}}-1+k_{f}} L_{2}(f)
$$

for all $f \in F$.

We will show that the following subcase never occurs. (The argument is identical to that given in Subcase 4.2.3.)

Subcase 4.3.3: $k_{1}=k_{Z_{1}}-2$.

Under the assumption $k_{2}=k_{Z_{2}}-1$, by Proposition 4.2.3 and equation (4.28),

$$
k_{1} \geq k_{2}-1=k_{Z_{2}}-2 \geq k_{Z_{1}}-2
$$

Under our assumption $k_{1}=k_{Z_{1}}-2$; therefore we must have equality everywhere in the above inequality:

$$
k_{1}=k_{2}-1=k_{Z_{2}}-2=k_{Z_{1}}-2
$$

By assumption, if $k_{Z_{2}}=k_{Z_{1}}$ then $E_{1}-E_{2}>0$. By Proposition 4.2.3, $k_{1} \geq k_{2}$, contradicting equation (4.41).

To complete Subcase 4.3 we need to argue that Subcases 4.3.1,4.3.2 and 4.3.3 are exhaustive. By equation (4.40), $k_{1} \geq k_{Z_{1}}-2$. It follows that the three subcases are exhaustive for subcase 4.3. Therefore, for $f \in F$,

$$
\left\|f_{s} \phi^{\prime}(f)-f_{s} \psi^{\prime}(f)\right\|<7 \varepsilon
$$

Subcase 4.4: $k_{Z_{2}}>k_{2}$ and if $k_{Z_{2}}=k_{2}+1$ then $r_{Z_{2}}>0$. 
By the results of Section 4.1, Subcase 3.3,

$$
f_{s} \psi^{\prime}=\iota_{0, Z_{2}}^{\prime} \iota_{0}^{k_{Z_{1}}-\left(k_{2}-k_{f}\right)-1} \iota_{\tau_{2}} \iota_{a_{1}}^{k_{2}-k_{f}} \iota_{a_{1}}^{k_{f}} L_{2}
$$

Subcase 4.4.1: $k_{2} \geq k_{f}$.

There are three further subcases.

Subcase 4.4.1.1: $k_{1}=k_{2}-k_{f}$. Then

$$
f_{s} \phi^{\prime}=\iota_{0, r Z_{2}}^{\prime} \iota_{0}^{k_{Z_{1}}-\left(k_{2}-k_{f}\right)-1} \iota_{r_{1}} \iota_{a_{1}}^{k_{2}-k_{f}} L_{1}
$$

By equation (4.39), for $f \in F$,

$$
\left\|\iota_{r_{2}} \iota_{a_{1}}^{k_{2}-k_{f}} \iota_{a_{1}}^{k_{f}} L_{2}(f)-\iota_{r_{1}} \iota_{a_{1}}^{k_{2}-k_{f}} L_{1}(f)\right\|<7 \varepsilon
$$

The actions of $\iota_{0, r_{2}}^{\prime}$ and $\iota_{0}$ on $\iota_{0}^{p} \iota_{r_{1}} \iota_{a_{1}}^{k_{2}-k_{f}} \iota_{a_{1}}^{k_{f}} L_{2}$ and $\iota_{0}^{p} \iota_{\tau_{1}} \iota_{a_{1}}^{k_{2}-k_{f}} L_{1}$ for $p \geq 0$ are the same - they are given by shifting by $V$, and conjugating by the trivial unitary $U(0, \ldots, 0)$. It follows that, for $f \in F$,

$$
\left\|f_{s} \psi^{\prime}(f)-f_{s} \phi^{\prime}(f)\right\|<7 \varepsilon
$$

Subcase 4.4.1.2: $k_{1}>k_{2}-k_{f}$.

By the construction given in Section 4.1, Subcase 3.3,

$$
\begin{aligned}
& f_{s} \phi^{\prime} \\
& \quad=\iota_{0, r Z_{2}}^{\prime} \iota_{0}^{k_{Z_{1}}-\left(k_{1}-k_{f}\right)-1} \iota_{r_{1}} \iota_{a_{1}}^{k_{1}-k_{f}} L_{1} \\
& \quad=\iota_{0, r Z_{2}}^{\prime} \iota_{0}^{k_{Z_{1}}-\left(k_{1}-k_{f}\right)-1} \iota_{\tau_{1}} \iota_{a_{1}}^{k_{1}-\left(1+k_{2}-k_{f}\right)} \iota_{a_{1}}^{1+k_{2}-k_{f}} L_{1} .
\end{aligned}
$$

By equation (4.38),

$$
\left\|\iota_{a_{1}}^{1+k_{2}-k_{f}} L_{1}(f)-\iota_{r_{2}} \iota_{a_{1}}^{k_{2}-k_{f}} \iota_{a_{1}}^{k_{f}} L_{2}(f)\right\|<7 \varepsilon .
$$


Comparing with equation (4.42), to find an estimate for the difference between $f_{s} \psi^{\prime}(f)$ and $f_{s} \phi^{\prime}(f)$ it is sufficient to prove that the unitary implementing the action of $\iota_{a_{1}}$ on $\iota_{a_{1}}^{1+k_{2}-k_{f}+p} L_{1}$, for $p \geq 0$ and the unitary implementing the action of $\iota_{\tau_{1}}$ on $\iota_{a_{1}}^{k_{1}-k_{f}} L_{1}$ are close to being trivial. By Remark 4.1.3, the unitary implementing the action of $\iota_{a_{1}}$ on $\iota_{a_{1}}^{1+k_{2}-k_{f}+p} L_{1}$ is given by

$$
U\left(s_{1-E_{1}+\left(2+k_{2}-k_{f}+p\right) a_{1}}, \ldots, s_{-E_{1}+\left(3+k_{2}-k_{f}+p\right) a_{1}}\right) \text {. }
$$

As $\left|s_{i}-0\right|<\delta$ for $i=F_{1}, F_{1}-1, \ldots, F_{1}-Z+1$ (by equation 4.17), it suffices to show that for $p=0$ the first subscript of $s_{i}$ appearing in unitary above is at least as large as $F_{1}-Z+1$. This follows from the calculation below:

$$
\begin{aligned}
1-E_{1}+\left(2+k_{2}-k_{f}\right) a_{1} & =1-E_{1}+2 a_{1}+k_{2} a_{1}-k_{f} a_{1} \\
& =1-\left(a_{1} k_{f}+E_{1}-E_{2}\right)-E_{2}+2 a_{1}+a_{1} k_{2} \\
& =1-(l-1)+\left(a_{1}-E_{2}\right)+a_{1} k_{2}+r_{2}-r_{2}+a_{1} \\
& =F_{2}-l+2+\left(a_{1}-r_{2}\right) \\
& \geq F_{2}-l+2 \\
& =F_{1}-Z+1 .
\end{aligned}
$$

The unitary implementing the action of $\iota_{\tau_{1}}$ on $\iota_{a_{1}}^{k_{1}-k_{f}} L_{1}$ is given by

$$
U\left(s_{F_{1}-r_{1}+1}, \ldots, s_{F_{1}}, 0, \ldots, 0\right) \text {. }
$$

As $F_{1}-r_{1}+1=1-E_{1}+\left(2+k_{2}-k_{f}+p\right) a_{1}$ for $p=k_{1}-k_{2}-1$, it follows that the above unitary is also close to being trivial.

We will show that the following subcase never occurs.

Subcase 4.4.1.3: $k_{1}<k_{2}-k_{f}$. 
As $k_{1} \geq k_{2}-1$, from the assumption made above, $k_{f}=0$. If $k_{f}=0$ then $k_{Z_{1}}=k_{Z_{2}}$, and it follows from Proposition 4.2.3 that $k_{1} \geq k_{2}$, contradicting our hypothesis.

Subcase 4.4.2: $k_{2}<k_{f}$.

We can reduce to the case where $k_{f}=k_{2}+1$ by the following argument which is very similar to the argument given in Proposition 4.2.2. By equation (4.17), $\left|t_{i}-1\right|<\delta$ for at least $a_{1} k_{f}+E_{1}-E_{2}$ values of $i$, and therefore $F_{2} \geq a_{1} k_{f}+E_{1}-E_{2}$. Then $a_{1} k_{2}+r_{2}=F_{2}-\left(a_{1}-E_{2}\right) \geq a_{1}\left(k_{f}-1\right)+E_{1}$, and therefore

$$
a_{1}\left(k_{2}-\left(k_{f}-1\right)\right) \geq E_{1}-r_{2}
$$

Since $r_{2}<a_{1}, k_{2} \geq k_{f}-1$ as required.

By Subcase 3.2,

$$
\iota_{\tau_{2}} \iota_{a_{1}}^{k_{2}} L_{2}(f) \sim_{7 \varepsilon} L_{1}(f)
$$

for all $f \in F$. We need to show that the unitary implementing the action of $\iota_{a_{1}}$ on $\iota_{a_{1}}^{p} L_{1}$ for $p \geq 0$, and the unitary implementing the action of $\iota_{\tau_{1}}$ on $\iota_{a_{1}}^{k_{1}} L_{1}$ are close to being trivial. As $\left|s_{i}-0\right|<\delta$ for $i=F_{1}, F_{1}-1, \ldots, F_{1}-Z+1$ (by equation (4.17)), it suffices to show that for $p=0$ the first subscript of $s_{i}$ appearing in the unitary above is at least as large as $F_{1}-Z+1$ :

$$
\begin{aligned}
F_{1}-Z+1 & =F_{2}-l+2 \\
& =F_{2}-\left(a_{1} k_{f}+E_{1}-E_{2}\right)+1 \\
& =F_{2}-\left(a_{1}\left(k_{2}+1\right)-E_{1}+E_{2}+1\right. \\
& =F_{2}-\left(a_{1}-E_{2}\right)-a_{1} k_{2}-E_{1}+1 \\
& =r_{2}-E_{1}+1 .
\end{aligned}
$$


As $1-E_{1}+a_{1} \geq 1-E_{1}+r_{2}$ it follow from the above calculation that the unitaries are close to being trivial. This completes the proof of Case 4.

Therefore, by Cases 1,2,3 and 4 we can conclude that

$$
\left\|f_{s} \psi^{\prime}(f)-f_{s} \phi^{\prime}(f)\right\|<7 \varepsilon
$$

for all $f \in F$. This completes the proof of Lemma 4.2.1.

\subsection{Endpoint considerations}

In this section we will find unitaries $U_{1}, U_{2} \in C[0,1] \otimes D_{2}$ that are close to each other (with respect to the finite set $F$ ) and twist the standard maps $\phi^{\prime}$ and $\psi^{\prime}$ into $A_{2}$.

By Lemma 4.1.1, there are unique sequences $1 \geq E s_{1} \geq E s_{2} \geq \cdots \geq$ $E s_{E F_{1}} \geq 0$ and $1 \geq E t_{1} \geq E t_{2} \geq \cdots \geq E t_{E F_{2}} \geq 0$, and unique pairs of positive integers $E E_{1}, E Z_{1}$ and $E E_{2}, E Z_{2}$ satisfying the conditions (i), (ii) and (iii) of the lemma for the maps $f_{\infty} \phi$ and $f_{\infty} \psi$ respectively. Applying the same lemma to the maps $f_{1} \phi$ and $f_{2} \psi$, we can find unique sequences $1 \geq s_{1} \geq s_{2} \geq \cdots \geq s_{F_{1}} \geq 0$ and $1 \geq t_{1} \geq t_{2} \geq \cdots \geq t_{F_{2}} \geq 0$, and unique pairs of positive integers $E_{1}, Z_{1}$ and $E_{2}, Z_{2}$ that together with the maps satisfy the conditions of the lemma.

There are two cases.

Case 1: $E Z_{1}=E Z_{2}$. 
By divisibility arguments if $E Z_{1}=E Z_{2}$ then

$$
E E_{1}=E E_{2} \text { and } E F_{1}=E F_{2}
$$

Lemma 4.3.1 If $E Z_{1}=E Z_{2}$ then $Z_{1}=Z_{2}$.

Proof. Consider the map $\tau: A_{1} \rightarrow M_{m}(\mathbb{C})$ defined as

$$
\tau(f)=\bigoplus_{1}^{\left(a_{2}+1\right) E E_{1}} e_{\infty} \bigoplus_{1}^{\left(a_{2}+1\right) E Z_{1}} 0(f) .
$$

Applying Lemma 4.1.1 to the map $\tau$, we find a unique sequence $1 \geq T s_{1} \geq$ $T s_{2} \geq \cdots \geq T s_{q} \geq 0$ and unique positive integers $T E$ and $T Z$ such that, together with the map $\tau$, they satisfy conditions (i), (ii) and (iii) of the lemma.

It is clear from the definition of the map $\tau$, that $T s_{\bar{i}} \in\{0,1\}$ for all $i=1, \ldots, q$. Find a positive integer $q^{\prime}$ such that $T s_{i}=1$ for $i=1, \ldots, q^{\prime}$ and $T s_{i}=0$ for $i=q^{\prime}+1, \ldots, q$. We claim that

$$
Z_{1}=T Z \text { and } E_{1}=T E .
$$

The argument relies essentially on the uniqueness property of Lemma 4.1.1. Since $f_{1} \phi$ is unitarily equivalent to $\bigoplus_{1}^{a_{2}+1} f_{\infty} \phi$, it is also unitarily equivalent to the map

$$
\bigoplus_{i=q^{\prime}+1}^{q} e_{T s_{i}} \bigoplus_{i=1}^{E F_{1}}\left(\bigoplus_{1}^{a_{2}+1} e_{E s_{i}}\right) \bigoplus_{i=1}^{q^{\prime}} e_{T s_{i}} \bigoplus_{1}^{T E} e_{\infty} \bigoplus_{1}^{T Z} 0
$$

Condition (i) of Lemma 4.1.1 applied to the map $\tau$ ensures that $T E<$ $a_{1}+1$. If $T Z>0$ then by condition (ii) of the same lemma, applied to map $\tau$, $q^{\prime}=0$. If $T Z>0$ then $E Z_{1}>0$, so by condition (ii) of Lemma 4.1.1 applied to the map $f_{\infty} \phi, E s_{i}<1$ for all $i$. Hence, all three conditions of Lemma 
4.1.1 are satisfied. By the uniqueness property of the lemma it follows that $E_{1}=T E$ and $Z_{1}=T Z$. The same argument then proves that $E_{2}=T E$ and $Z_{2}=T Z$. This completes the proof of the Lemma 4.3.1.

Let $\gamma: A_{1} \rightarrow C_{2}$ be a map in standard form defined by perturbing the eigenvalues of $f_{\infty} \phi$ so that they are distinct:

$$
\gamma=\operatorname{Std}\left(E s_{1}^{\prime}, \ldots, E s_{E F}^{\prime}, E E, E Z\right)
$$

where $1>E s_{1}^{\prime}>\cdots>E s_{E F}^{\prime}>0, E F=E F_{1}=E F_{2}, E Z=E Z_{1}=E Z_{2}$, and

$$
\left|E s_{i}^{\prime}-E s_{i}\right|<\delta
$$

Let $\left(f_{\infty} \phi\right)^{\prime},\left(f_{\infty} \psi\right)^{\prime}: A_{1} \rightarrow C_{2}$ denote the maps $f_{\infty} \phi$ and $f_{\infty} \psi$, respectively, in standard form. To complete the proof of Case 1 it is sufficient to find unitaries $U_{1 R}$ and $U_{2 R}$ that are close to each other such that the following relations hold at the right endpoint

$$
\begin{aligned}
\operatorname{Ad}_{U_{R}^{*}} f_{1} \phi^{\prime} & =\bigoplus_{1}^{a_{2}+1}\left(f_{\infty} \phi\right)^{\prime} \\
\operatorname{Ad}_{U_{1 R}^{*}} f_{\mathrm{I}} \psi^{\prime} & =\bigoplus_{1}^{a_{2}+1}\left(f_{\infty} \psi\right)^{\prime} .
\end{aligned}
$$

(A similar pair of unitaries must also be found for the left endpoint.) Let $E p_{j, i}, E q_{j, i}$ and $E p_{j, i}^{\prime}$ denote the support projections of the subrepresentations $e_{E s_{j}}, e_{E t_{j}}$ and $e_{E s_{j}^{\prime}}$ in the $i^{\prime}$ th direct summand of $\bigoplus_{1}^{a_{2}+1}\left(f_{\infty} \phi\right)^{\prime}, \bigoplus_{1}^{a_{2}+1}\left(f_{\infty} \psi\right)^{\prime}$ and $\bigoplus_{1}^{\alpha_{2}+1} \gamma$, respectively. By Proposition 4.1.2 as $\left|E s_{i}-E s_{i}^{\prime}\right|<\delta$ and $\left|E t_{i}-E s_{i}\right| \leq\left|E t_{i}-E s_{i}\right|+\left|E s_{i}-E s_{i}^{\prime}\right|<2 \delta$, there are unitaries $E U_{k, i} \in$ 
$C_{2}, k=1,2$ such that

$$
\begin{aligned}
& \operatorname{Ad}_{E U_{1, i}} E p_{j, i}=E p_{j, i}^{\prime} \\
& \operatorname{Ad}_{E U_{2, i}} E q_{j, i}=E p_{j, i}^{\prime}
\end{aligned}
$$

and

$$
\left\|E U_{k, i}-\mathrm{Id}\right\|<2 \pi \delta<\varepsilon .
$$

Let $\beta$ be a map in standard form unitarily equivalent to $a_{2}+1$ copies of $\gamma$ :

$$
\beta=\left(\bigoplus_{1}^{a_{2}+1} \gamma\right)^{\prime}
$$

(where $\left(\bigoplus_{1}^{a_{2}+1} \gamma\right)^{\prime}$ denotes the map $\bigoplus_{1}^{a_{2}+1} \gamma$ in standard form). By Lemma 4.1.1 there exists a unique sequence $1 \geq s_{1}^{\prime} \geq \cdots \geq s_{F}^{\prime} \geq 0$ and positive integers $E, Z$ that together with the map $\beta$ satisfy the conditions of the lemma. Recall from Section 4.2 that $f_{1} \phi^{\prime}$ and $f_{1} \psi^{\prime}$ denote the maps $\phi$ and $\psi$ in standard form at the right endpoint. In particular, $f_{1} \phi^{\prime}$ and $f_{1} \psi^{\prime}$ are unitarily equivalent to $\bigoplus_{1}^{a_{2}+1} f_{\infty} \phi$ and $\bigoplus_{1}^{a_{2}+1} f_{\infty} \psi$ respectively. Let $V$ be any unitary such that

$$
\operatorname{Ad}_{V}\left(\bigoplus_{1}^{a_{2}+1} \gamma\right)=\beta
$$

Let $p_{i}, q_{i}, p_{i}^{\prime}$ denote the support projections of the subrepresentations $e_{s_{i}}, e_{t_{i}}$ and $e_{s_{i}^{\prime}}$ of $f_{1} \phi^{\prime}, f_{1} \psi^{\prime}$ and $\beta$ respectively. Then $\operatorname{Ad}_{V}\left(E p_{j, i}^{\prime}\right)=p_{f(j, i)}^{\prime}$ for some bijection $f$. By Lemma 4.3.1, as $E Z_{1}=E Z_{2}=E Z, Z=Z_{1}=Z_{2}$. As $\beta, f_{1} \phi$ and $f_{1} \psi$ are all represented on the same Hilbert space, a simple counting argument then proves that $F_{1}=F_{2}=F$ and $E_{1}=E_{2}=E$. By equation (4.44) it follows that $\left|s_{i}-s_{i}^{\prime}\right|<\delta$ for $i=1, \ldots, F$. By Proposition 4.1.2 as 
$\left|s_{i}-s_{i}^{\prime}\right|<\delta$ and $\left|t_{i}-s_{i}^{\prime}\right|<2 \delta$ there exists a pair of unitaries $U_{k}^{\prime}, k=1,2$ such that

$$
\begin{aligned}
\operatorname{Ad}_{U_{1}^{\prime}} p_{i} & =p_{i}^{\prime} \\
\operatorname{Ad}_{U_{2}^{\prime}} q_{i} & =p_{i}^{\prime}
\end{aligned}
$$

and

$$
\left\|U_{k}^{\prime}-\mathrm{Id}\right\|<2 \pi \delta<\varepsilon
$$

Let

$$
U_{k R}=U_{k}^{\prime *} V \bigoplus_{i=1}^{a_{2}+1} E U_{k, i}, k=1,2
$$

where $\bigoplus_{i=1}^{a_{2}+1} U_{k, i} \in D_{2}$ by the embedding $\bigoplus_{1}^{a_{2}+1} C_{2} \hookrightarrow D_{2}$. Then by equations (4.45) and (4.47),

$$
\operatorname{Ad}_{U_{1 R}} E p_{j, i}=p_{f(j, i)}, \operatorname{Ad}_{U_{2 R}} E q_{j, i}=q_{f(j, i)}
$$

and by equations (4.46) and (4.48),

$$
\left\|U_{1 R}-U_{2 R}\right\|<4 \varepsilon
$$

By the above computations,

$$
\begin{aligned}
& \operatorname{Ad}_{U_{1 R^{*}}} f_{1} \phi^{\prime}=\oplus_{1}^{a_{2}+1}\left(f_{\infty} \phi\right)^{\prime} \\
& \operatorname{Ad}_{U_{2 R^{*}}} f_{1} \psi^{\prime}=\oplus_{1}^{a_{2}+1}\left(f_{\infty} \psi\right)^{\prime} .
\end{aligned}
$$

(By a similar argument we can find unitaries $U_{1 L}$ and $U_{2 L}$ for the left endpoint of $A_{2}$.) Finally, define unitaries $U_{1}$ and $U_{2}$ by joining $U_{1 R}^{*}$ to $U_{1 L}^{*}$ and $U_{2 R}^{*}$ to $U_{2 L}^{*}$ by unitary paths over the unit interval that are at least as close at every 
intermediate point as at the endpoints. Therefore by equations (4.43) and (4.49), for $f \in F$,

$$
\begin{aligned}
\left\|\operatorname{Ad}_{U_{1}} \phi^{\prime}(f)-\operatorname{Ad}_{U_{2}} \psi^{\prime}(f)\right\| & \leq\left\|\operatorname{Ad}_{U_{2}^{*} U_{1}} \phi^{\prime}(f)-\phi^{\prime}(f)\right\|+\left\|\phi^{\prime}(f)-\psi^{\prime}(f)\right\| \\
& \leq 8 \varepsilon+7 \varepsilon=15 \varepsilon .
\end{aligned}
$$

This completes the proof of Case 1 .

Case 2. $E Z_{1}<E Z_{2}$.

The following two propositions are well known and will be used in the proof of Lemma 4.3.2.

Proposition 4.3.1 If $P$ and $Q$ are projections in a matrix algebra such that $\|P-Q\|<\delta<1 / 2$ then there exists a unitary $U$ such that $\|U-I d\|<3 \delta$ and $U P U^{*}=Q$.

Proposition 4.3.2 Let $\alpha$ and $\beta$ be two finite dimensional representations of $M_{n}(\mathbb{C})$ such that $\alpha\left(p_{i}\right)=\beta\left(p_{i}\right)$, where $\left\{p_{i}\right\}_{1}^{n}$ are a complete set of orthogonal minimal projections. Then there exists a unitary path to the identity, $U_{t}$ such that $\operatorname{Ad}_{U_{I}} \alpha=\beta$ and $\operatorname{Ad}_{U_{t}} \alpha(d)=\beta(d)$ for all diagonal elements $d \in M_{n}(\mathbb{C})$ (diagonal with respect to the set of projections $\left\{p_{i}\right\}_{1}^{n}$ ).

Lemma 4.3.2 Let $F \subset A_{1}$ be a finite set containing the canonical selfadjoint element $h, \varepsilon>0$ and $\delta$ be defined as in (4-3). Given any sequence of positive real numbers $1 \geq s_{1} \geq s_{2} \geq \cdots \geq s_{F} \geq 0$ such that $\left|s_{i}-0\right|<\delta$ or $\left|s_{i}-1\right|<\delta$ for all $i=1, \ldots, F$, positive integers $E, Z \geq 0$, and two maps $\alpha, \beta: A_{1} \rightarrow M_{n}(\mathbb{C})$ unitarily equivalent to $e_{s_{1}} \oplus \cdots \oplus e_{s_{F}} \bigoplus_{1}^{E} e_{\infty} \bigoplus_{1}^{Z} 0$ such that $\|\alpha(f)-\beta(f)\|<\varepsilon / 2$ for $f \in F$, there exists a unitary path $U_{t}$ to the identity such that $\operatorname{Ad}_{U_{1}} \alpha=\beta$ and $\left\|\operatorname{Ad}_{U_{z}} \alpha(f)-\beta(f)\right\|<19 \varepsilon$ for $f \in F$. 
Proof. Let

$$
\begin{aligned}
& \alpha=T_{1} e_{s_{1}} \oplus \cdots \oplus e_{s_{F}} \bigoplus_{1}^{E} e_{\infty} \bigoplus_{1}^{Z} 0 T_{1}^{*} \\
& \beta=T_{2} e_{s_{1}} \oplus \cdots \oplus e_{s_{F}} \bigoplus_{1}^{E} e_{\infty} \bigoplus_{1}^{Z} 0 T_{2}^{*} \\
& \gamma=e_{s_{1}} \oplus \cdots \oplus e_{s_{F}} \bigoplus_{1}^{E} e_{\infty} \bigoplus_{1}^{Z} 0
\end{aligned}
$$

for some pair of unitaries $T_{1}$ and $T_{2}$. Suppose that $\left|s_{i}-1\right|<\delta$ for $i=1, \ldots, q$ and $\left|s_{i}-0\right|<\delta$ for $i=q+1, \ldots, F$. Let

$$
s_{i}^{\prime}=\left\{\begin{array}{lll}
1 & \text { for } & i \leq q \\
0 & \text { for } & i>q .
\end{array}\right.
$$

Then define maps $\alpha^{\prime}$ and $\beta^{\prime}$ as follows

$$
\begin{aligned}
& \alpha^{\prime}=T_{1} e_{s_{1}^{\prime}} \oplus \cdots \oplus e_{s_{F}^{\prime}} \bigoplus_{1}^{E} e_{\infty} \bigoplus_{1}^{Z} 0 T_{1}^{*} \\
& \beta^{\prime}=T_{2} e_{s_{1}^{\prime}} \oplus \cdots \oplus e_{s_{F}^{\prime}} \bigoplus_{1}^{E} e_{\infty} \bigoplus_{1}^{Z} 0 T_{2}^{*} .
\end{aligned}
$$

Let $P=\alpha^{\prime}(h)$ and $Q=\beta^{\prime}(h)$, then $P$ and $Q$ are projections such that

$$
\begin{aligned}
\|P-Q\| & \leq\|P-\alpha(h)\|+\|\alpha(h)-\beta(h)\|+\|\beta(h)-Q\| \\
& <3 \varepsilon / 2 .
\end{aligned}
$$

By Proposition 4.3.1 there is a unitary path $U_{t}^{\prime}$ such that

$$
\left\|U_{t}^{\prime}-\mathrm{Id}\right\|<9 \varepsilon
$$

for all $t \in[0,1]$ and

$$
\operatorname{Ad}_{U_{\mathrm{I}}^{\prime}} P=Q .
$$


As $e_{1}(h) e_{s}(f)=e_{s}(f) e_{1}(h)=e_{s}(f)$, for all $s \in[0,1]$ and $f \in A_{1}$, the subrepresentations $T_{1} e_{s_{i}} T_{1}^{*}$ and $T_{1} e_{\infty} T_{1}^{*}$ of $\alpha$ for $i \leq q$ are supported on $P$. Therefore, their unitary conjugates by $U_{1}^{r}-\operatorname{Ad}_{U_{1}^{\prime}} T_{1} e_{s_{i}} T_{1}^{*}$ and $\operatorname{Ad}_{U_{1}^{r}} T_{1} e_{\infty} T_{1}^{*}$ - are supported on $Q$. Similarly, for $i>q$ the cut-down (of the subrepresentation $\left.e_{s_{i}}\right) \operatorname{Ad}_{U_{1}^{\prime}} T_{1} e_{0}(h) e_{s_{i}} e_{o}(h) T_{1}^{*}$ is supported on $Q$ as well. The subrepresentations of $\beta, T_{2} e_{s_{i}} T_{2}^{*}$ and $T_{2} e_{\infty} T_{2}^{*}$, for $i \leq q$ and the cut-down (of the subrepresentation $\left.e_{s_{i}}\right) T_{2} e_{0}(h) e_{s_{i}} e_{o}(h) T_{2}^{*}$ for $i>q$ are supported on $Q$. Therefore there is a unitary $S_{1}$ supported on $Q$ such that

$$
\begin{aligned}
\operatorname{Ad}_{S_{1}} T_{2} e_{s_{i}} T_{2}^{*} & =\operatorname{Ad}_{U_{1}^{\prime}} T_{1} e_{s_{i}} T_{1}^{*} \text { for } i \leq q \\
\operatorname{Ad}_{S_{1}} T_{2} e_{\infty} T_{2}^{*} & =\operatorname{Ad}_{U_{1}^{r}} T_{1} e_{\infty} T_{1}^{*} \\
\operatorname{Ad}_{S_{1}} T_{2} e_{0}(h) e_{s_{i}} e_{o}(h) T_{2}^{*} & =\operatorname{Ad}_{U_{1}^{\prime}} T_{1} e_{0}(h) e_{s_{i}} e_{o}(h) T_{1}^{*} \text { for } i>q
\end{aligned}
$$

The cut-downs $\operatorname{Ad}_{U_{1}} T_{1} e_{0}(h)^{\perp} e_{s_{i}} e_{o}(h)^{\perp} T_{1}^{*}$ and $T_{2} e_{0}(h)^{\perp} e_{s_{i}} e_{o}(h)^{\perp} T_{2}^{*}$ for $i>q$ are supported on $Q^{\perp}$, so there is a unitary $S_{1}^{\perp}$ such that for $i>q$,

$$
\operatorname{Ad}_{U_{1}^{\prime}} T_{1} e_{0}(h)^{\perp} e_{s_{i}} e_{o}(h)^{\perp} T_{1}^{*}=\operatorname{Ad}_{S_{1}^{\perp}} T_{2} e_{0}(h)^{\perp} e_{s_{i}} e_{o}(h)^{\perp} T_{2}^{*}
$$

As the unitaries $S_{1}$ and $S_{1}^{\perp}$ are supported on orthogonal projections we may rewrite equations $(4.52)-(4.55)$ as

$$
\begin{aligned}
& \operatorname{Ad}_{S_{1}^{\perp} S_{1}} T_{2} e_{s_{i}} T_{2}^{*}=\operatorname{Ad}_{U_{1}^{r}} T_{1} e_{s_{i}} T_{1}^{*} \\
& \operatorname{Ad}_{S_{1}^{\perp} S_{1}} T_{2} e_{\infty} T_{2}^{*}=\operatorname{Ad}_{U_{1}^{\prime}} T_{1} e_{\infty} T_{1}^{*}
\end{aligned}
$$

for $i \leq q$ and

$$
\begin{aligned}
\operatorname{Ad}_{S_{1}^{\perp} S_{1}} T_{2} e_{0}(h) e_{s_{i}} e_{o}(h) T_{2}^{*} & =\operatorname{Ad}_{U_{1}^{\prime}} T_{1} e_{0}(h) e_{s_{i}} e_{o}(h) T_{1}^{*} \\
\operatorname{Ad}_{S_{1}^{\perp} S_{1}} T_{2} e_{0}(h)^{\perp} e_{s_{i}} e_{o}(h)^{\perp} T_{2}^{*} & =\operatorname{Ad}_{U_{1}^{\prime}} T_{1} e_{0}(h)^{\perp} e_{s_{i}} e_{o}(h)^{\perp} T_{1}^{*}
\end{aligned}
$$


for $i>q$. In the argument that follows we will identify $M_{a_{1}+1}(\mathbb{C})$ with the range of the subrepresentation $e_{s_{i}}$ of the map $\gamma$. We will view $e_{0}(h)$ and $e_{0}(h)^{\perp}$ as rank $a_{1}$ and rank 1 projections inside $M_{a_{1}+1}(\mathbb{C})$. By equation (4.58), for every subprojection $p \leq e_{0}(h)$,

$$
\operatorname{Ad}_{S_{1}^{\perp} S_{1}} T_{2} p T_{2}^{*}=\operatorname{Ad}_{U_{1}^{\prime}} T_{1} p T_{1}^{*} \text { for } i>q
$$

By equation (4.59), for the rank 1 projection $e_{0}(h)^{\perp}$ a similar relation holds,

$$
\operatorname{Ad}_{S_{1}^{\perp} S_{1}} T_{2} e_{0}(h)^{\perp} T_{2}^{*}=\operatorname{Ad}_{U_{1}^{\prime}} T_{1} e_{0}(h)^{\perp} T_{1}^{*} \text { for } i>q
$$

Let $\left\{p_{1}, \ldots, p_{a_{1}}\right\}$ be a complete set of orthogonal minimal subprojections of $e_{0}(h)$, and let $p_{a_{1}+1}=e_{0}(h)^{\perp}$. Then by equations (4.60) and (4.61),

$$
\operatorname{Ad}_{S_{1}^{\perp} S_{1}} T_{2} p_{j} T_{2}^{*}=\operatorname{Ad}_{U_{1}^{\prime}} T_{1} p_{j} T_{1}^{*} \text { for } i>q \text { and } 1 \leq j \leq a_{1}+1
$$

Therefore, by Proposition 4.3.2 there is a unitary path $V_{t}$ to the identity, supported on $\operatorname{Ad}_{S_{1}^{\perp} S_{1}} T_{2}$ (Id) $T_{2}^{*}$ (where Id is the identity element of $M_{a_{1}+1}(\mathbb{C})$ ) such that

$$
\operatorname{Ad}_{V_{1}} \operatorname{Ad}_{U_{1}^{\prime}} T_{1} g T_{1}^{*}=\operatorname{Ad}_{S_{1}^{\perp} S_{1}} T_{2} g T_{2}^{*}
$$

for all $g \in M_{a_{1}+1}(\mathbb{C})$. In particular,

$$
\operatorname{Ad}_{V_{1}} \operatorname{Ad}_{U_{1}^{\prime}} T_{1} e_{s_{i}} T_{1}^{*}=\operatorname{Ad}_{S_{1}^{\perp} S_{1}} T_{2} e_{s_{i}} T_{2}^{*} \text { for } i>q
$$

Proposition 4.3.2 also ensures that

$$
\operatorname{Ad}_{V_{\imath}} \operatorname{Ad}_{U_{t}^{\prime}} T_{1} g T_{1}^{*}=\operatorname{Ad}_{S_{1}^{\perp} S_{1}} T_{2} g T_{2}^{*}, t \in[0,1]
$$

for all $g \in M_{a_{1}+1}(\mathbb{C})$ which are diagonal with respect to the set of projections $\left\{p_{i}\right\}_{i=1}^{a_{1}+1}$, and in particular $\operatorname{Ad}_{V_{i}} \operatorname{Ad}_{U_{1}^{\prime}} T_{1} e_{o}(f) T_{1}^{*}=\operatorname{Ad}_{S_{1}^{\perp} S_{1}} T_{2} e_{0}(f) T_{2}^{*}$ for $t \in$ 
$[0,1]$ and $f \in F$. For $f \in F$ and $i>q,\left\|e_{s_{i}}(f)-e_{0}(f)\right\|<\varepsilon$. Therefore, for $f \in F$ and $i>q$,

$$
\begin{aligned}
\left\|\operatorname{Ad}_{V_{t}} \operatorname{Ad}_{U_{1}^{\prime}} T_{1} e_{s_{i}}(f) T_{1}^{*}-\operatorname{Ad}_{S_{1}^{\perp} S_{1}} T_{2} e_{s_{i}}(f) T_{2}^{*}\right\| \\
\leq \quad\left\|\operatorname{Ad}_{V_{t}} \operatorname{Ad}_{U_{1}^{\prime}} T_{1} e_{s_{i}}(f) T_{1}^{*}-\operatorname{Ad}_{V_{t}} \operatorname{Ad}_{U_{1}^{\prime}} T_{1} e_{0}(f) T_{1}^{*}\right\| \\
\quad+\left\|\operatorname{Ad}_{V_{t}} \operatorname{Ad}_{U_{1}^{\prime}} T_{1} e_{0}(f) T_{1}^{*}-\operatorname{Ad}_{S_{1}^{\perp} S_{1}} T_{2} e_{s_{i}}(f) T_{2}^{*}\right\| \\
\leq 2 \varepsilon .
\end{aligned}
$$

It is immediate from the definition of $\alpha^{\prime}$ and $\beta^{\prime}$ that

$$
\begin{aligned}
& \alpha^{\prime}(f)=e_{\infty}(f) P \\
& \beta^{\prime}(f)=e_{\infty}(f) Q
\end{aligned}
$$

for all $f \in A_{1}$ (as $e_{\infty}(f) \in \mathbb{C}$, the expression on the right-hand side of both equations should be interpreted as the scalar multiple of a projection). Therefore $\left\|\beta(f)-e_{\infty}(f) Q\right\|<\varepsilon$ for all $f \in F$, and since $\operatorname{Ad}_{S_{t}^{\perp} S_{t}} e_{\infty}(f) Q=$ $e_{\infty}(f) Q$

$$
\left\|A d_{S_{t}^{\perp} S_{t}} \beta(f)-\beta(f)\right\|<2 \varepsilon
$$

for all $f \in F$, where $S_{t}$ and $S_{t}^{\perp}$ are unitary paths to the identity supported on $Q$ and $Q^{\perp}$ respectively.

Let $U_{t}$ be the unitary path formed by concatenating the paths $U_{t}^{\prime}, V_{t}$ and $\left(S_{t}^{\perp} S_{t}\right)^{*}$

$$
U_{t}=\left\{\begin{array}{rll}
U_{3 t}^{\prime} & \text { for } & t \in[0,1 / 3] \\
V_{3(t-1 / 3)} U_{1}^{\prime} & \text { for } & t \in[1 / 3,2 / 3] \\
\left(S_{3(t-2 / 3)}^{\perp} S_{3(t-2 / 3)}\right)^{*} V_{1} U_{1}^{\prime} & \text { for } & t \in[2 / 3,1]
\end{array}\right.
$$


Let us check that $U_{t}$ satisfies the conclusions of the lemma. By equation (4.51), as $\|\alpha(f)-\beta(f)\|<\varepsilon_{\text {, }}$

$$
\begin{aligned}
\left\|\operatorname{Ad}_{U_{t}^{\prime}} \alpha(f)-\beta(f)\right\| & \leq\left\|\operatorname{Ad}_{U_{t}^{\prime}} \alpha(f)-\alpha(f)\right\|+\|\alpha(f)-\beta(f)\| \\
& <18 \varepsilon+\varepsilon=19 \varepsilon .
\end{aligned}
$$

By equations (4.56),(4.57) and (4.62),

$$
\operatorname{Ad}_{V_{1}} \operatorname{Ad}_{U_{1}^{\prime}} T_{1} e_{s_{i}} T_{1}^{*}=\operatorname{Ad}_{S_{1}^{1} S_{1}} T_{2} e_{s_{i}} T_{2}^{*}
$$

for $i=1, \ldots, F$ and $i=\infty$, or equivalently,

$$
\operatorname{Ad}_{V_{1} U_{1}^{\prime}} \alpha=\operatorname{Ad}_{S_{1}^{L} S_{1}} \beta
$$

and therefore,

$$
\operatorname{Ad}_{U_{1}} \alpha=\beta
$$

By equation (4.63), for $f \in F$,

$$
\left\|\operatorname{Ad}_{V_{t}}\left(\operatorname{Ad}_{U_{1}^{\prime}} \alpha(f)\right)-\operatorname{Ad}_{S_{1}^{\perp} S_{1}} \beta(f)\right\|<2 \varepsilon .
$$

By equations (4.67) and (4.64), for $f \in F$, and $t \in[0,1]$,

$$
\begin{aligned}
\left\|\operatorname{Ad}_{V_{t}} \operatorname{Ad}_{U_{1}^{\prime}} \alpha(f)-\beta(f)\right\| \leq & \left\|\operatorname{Ad}_{V_{t}} \operatorname{Ad}_{U_{1}^{\prime}} \alpha(f)-\operatorname{Ad}_{S_{\frac{1}{1}} S_{1}} \beta(f)\right\| \\
& +\left\|\operatorname{Ad}_{S_{1}^{\perp} S_{1}} \beta(f)-\beta(f)\right\| \\
\leq & 4 \varepsilon .
\end{aligned}
$$

Evaluating the above equation at $t=1$, and by equation (4.64), for $f \in F$ and $t \in[0,1]$,

$$
\left\|\operatorname{Ad}_{\left(S_{t}^{\perp} S_{t}\right)} \cdot \operatorname{Ad}_{V_{1}} \operatorname{Ad}_{U_{1}^{\prime}} \alpha(f)-\beta(f)\right\|=\left\|\operatorname{Ad}_{V_{1}} \operatorname{Ad}_{U_{1}^{\prime}} \alpha(f)-\operatorname{Ad}_{S_{t}^{\perp} S_{t}} \beta(f)\right\|
$$




$$
\begin{aligned}
\leq & \left\|\operatorname{Ad}_{V_{1}} \operatorname{Ad}_{U_{1}^{r}} \alpha(f)-\beta(f)\right\| \\
& +\left\|\beta(f)-\operatorname{Ad}_{S_{t}^{+} S_{t}} \beta(f)\right\| \\
\leq & 4 \varepsilon+2 \varepsilon=6 \varepsilon .
\end{aligned}
$$

Therefore, by equations $(4.66),(4.65), 4.68)$ and $(4.69) U_{t}$ satisfies the conclusions of the lemma. This completes the proof of Lemma 4.3.2.

Lemma 4.3.3 Let $\alpha=\operatorname{Std}\left(s_{1}, \ldots, s_{F}, E, Z\right)$ where $1 \geq s_{1} \geq \cdots \geq s_{F} \geq 0$, $E$ and $Z$ together with $\alpha$ satisfy the conclusions of Lemma 4.1.1. Let $p$ be the support projection for the subrepresentation $e_{s_{k+1}} \oplus \cdots \oplus e_{s_{F}}$ of $\alpha$. If $\beta=\operatorname{Std}\left(s_{1}^{\prime}, \ldots, s_{k}^{\prime}, s_{k+1}, \ldots, s_{F}, E, Z\right)$ is any other map in standard form where $s_{i}^{\prime}=1$ for $i=1, \ldots, k\left(1 \geq s_{1}^{\prime} \geq \ldots s_{k}^{\prime} \geq s_{k+1} \geq \ldots s_{F} \geq 0, E\right.$ and $Z$ together with $\beta$ may no longer satisfy the conclusions of Lemma 4.1.1) then $p \alpha p=p \beta p$.

Proof. Suppose that $s_{i}=1$ for $i=1, \ldots, q$ and $s_{i}<1$ for $i=q+1, \ldots, k$. Let

$$
s_{i, \varepsilon}^{\prime \prime}=\left\{\begin{aligned}
\max \left(1-\varepsilon, s_{k+1}\right) & \text { for } 1+q \leq i \leq k \\
s_{i} & \text { otherwise. }
\end{aligned}\right.
$$

Let $\beta_{\varepsilon}$ be the map in standard form,

$$
\beta_{\varepsilon}=\operatorname{Std}\left(s_{1, \varepsilon}^{\prime \prime}, \ldots, s_{F, \varepsilon}^{\prime \prime}, E, Z\right)
$$

Then, for $\varepsilon>0,1 \geq s_{1, \varepsilon}^{\prime \prime} \geq \cdots \geq s_{F, \varepsilon}^{\prime \prime}, E$ and $Z$ together with the map $\beta_{\varepsilon}$ satisfy the conditions of Lemma 4.1.1. By Proposition 4.1.2, $p \alpha p=p \beta_{\varepsilon} p$ for $\varepsilon>0$. The standard maps are continuous, and therefore $p \alpha p=p \beta_{0} p$. As $\beta_{o}=\beta$ it follows that $p \alpha p=p \beta p$. 
Proposition 4.3.3 Let $1 \geq s_{1} \geq \cdots \geq s_{F_{1}} \geq 0$ and $1 \geq t_{1} \geq \cdots \geq t_{F_{2}} \geq 0$ be sequences of real numbers and $E_{1}, Z_{1}$ and $E_{2}, Z_{2}$ (with $Z_{2} \geq Z_{1}$ ) be pairs of positive integers satisfying the conclusions of Lemma 4.1 .1 for the maps $\alpha=\operatorname{Std}\left(s_{1}, \ldots, s_{F_{1}}, E_{1}, Z_{1}\right)$ and $\beta=\operatorname{Std}\left(t_{1}, \ldots, t_{F_{2}}, E_{2}, Z_{2}\right)$ respectively. If the eigenvalues of $\alpha(h)$ and $\beta(h)$ can be matched within $\delta$ then there is an integer $d \geq 0$ and a small perturbation $t_{1}^{\prime} \geq \cdots \geq t_{F_{2}}^{\prime} \geq 0$ such that $t_{i}^{\prime}=$ $1,\left|t_{i}-t_{i}^{\prime}\right|<\delta$ for $i=1, \ldots, d$ and $t_{i}^{\prime}=t_{i}$ for $i>d$. We can choose $d$ such that applying Lemma 4.1 .1 to the map $\gamma=\operatorname{Std}\left(t_{1}^{\prime}, \ldots, t_{F_{2}}^{\prime}, E_{2}, Z_{2}\right)$ yields a sequence $s_{1}^{\prime} \geq \cdots \geq s_{F_{1}}^{\prime} \geq 0$ such that $\left|s_{i}-s_{i}^{\prime}\right|<2 \delta$ for $i=1, \ldots, F_{1}$ and $\gamma=\operatorname{Std}\left(s_{1}^{\prime}, \ldots, s_{F_{1}}^{\prime}, E_{1}, Z_{1}\right)$. Furthermore, the sequence $s_{1}^{\prime} \geq \cdots \geq s_{F_{1}}^{\prime} \geq 0$ and the pair of positive integers $E_{1}, Z_{1}$, together with the map $\gamma$ satisfies the conclusions of Lemma 4.1.1.

Proof. The proof proceeds in two steps. First we will define the integer $d$ and demonstrate that the perturbation of the sequence $t_{i}$ yields (under the application of Lemma 4.1.1) a sequence $s_{i}^{\prime}$ that is close to the given sequence $s_{i}$. The second step is to show that $\operatorname{Std}\left(t_{1}^{\prime}, \ldots, t_{F_{2}}^{\prime}, E_{2}, Z_{2}\right)=$ $\operatorname{Std}\left(s_{1}^{\prime}, \ldots, s_{F_{1}}^{\prime}, E_{1}, Z_{1}\right)$.

The first step has three cases.

Case 1: $Z_{1}<a_{1}+1-E_{1}$ and $Z_{2}<a_{1}+1-E_{2}$.

By equation (4.13), $Z_{2}-Z_{1}=E_{1}-E_{2}$ and by equation (4.14), $\left|t_{i}-1\right|<\delta$ for $i=1, \ldots, Z$. Let $d=Z$. Define $t_{i}^{\prime}$ and $s_{i}^{\prime}$ as follows:

$$
t_{i}^{\prime}=\left\{\begin{array}{cll}
1 & \text { for } & i \leq Z \\
t_{i} & \text { otherwise } &
\end{array}\right.
$$


and

$$
s_{i}^{\prime}=\left\{\begin{array}{rll}
0 & \text { for } & F \geq i \geq F-Z+1 \\
t_{Z+i} & \text { for } & F-Z \geq i \geq 1 .
\end{array}\right.
$$

We need to check that Lemma 4.1.1 applied to the map $\operatorname{Std}\left(t_{1}^{\prime}, \ldots, t_{F_{2}}^{\prime}, E_{2}, Z_{2}\right)$ yields the sequence of real numbers $\left\{s_{i}^{\prime}\right\}_{i=1}^{F_{1}}$ and pair of positive integers $E_{1}, Z_{1}$. As $E_{1}<a_{1}+1$ and $E_{1}<a_{1}$ when $Z_{1}>0$ (by the hypothesis of the proposition), conditions (i) and (ii) of Lemma 4.1.1 are satisfied. To verify condition (iii), it is enough to check that $\operatorname{Std}\left(t_{1}^{\prime}, \ldots, t_{F_{2}}^{\prime}, E_{2}, Z_{2}\right)$ is unitarily equivalent to $e_{s_{1}^{\prime}} \oplus \cdots \oplus e_{s_{F_{1}}^{\prime}} \bigoplus_{1}^{E_{1}} e_{\infty} \bigoplus_{1}^{Z_{1}} 0$. This is an easy calculation (a similar, more difficult calculation will be performed in Case 2).

Case 2: $Z_{1}<a_{1}+1-E_{1}$ and $Z_{2} \geq a_{1}+1-E_{2}$.

Let $d=l-1$. Define $t_{i}^{\prime}$ and $s_{i}^{\prime}$ as follows:

$$
t_{i}^{\prime}=\left\{\begin{array}{lll}
1 & \text { for } & 1 \leq i \leq l-1 \\
t_{i} & \text { otherwise }
\end{array}\right.
$$

where $l-1=a_{1}\left(k_{Z_{2}}+1\right)+E_{1}-E_{2}$ and

$$
s_{i}^{\prime}=\left\{\begin{array}{rll}
0 & \text { for } & F_{1} \geq i \geq F_{1}-Z+1 \\
& \text { for } & F_{1}-Z \geq i \geq 1
\end{array}\right.
$$

We need to check that the sequence of real numbers $\left\{s_{i}^{\prime}\right\}_{i=1}^{F_{1}}$ and pair of positive integers $E_{1}, Z_{1}$ satisfies conditions (i), (ii) and (iii) of Lemma 4.1.1 applied to the map $\operatorname{Std}\left(t_{1}^{\prime}, \ldots, t_{F_{2}}^{\prime}, E_{2}, Z_{2}\right)$. It is clear from the hypothesis of the proposition that conditions (i) and (ii) are satisfied. We will show that $\operatorname{Std}\left(t_{1}^{\prime}, \ldots, t_{F_{2}}^{\prime}, E_{2}, Z_{2}\right)$ is unitarily equivalent to $e_{s_{1}^{\prime}} \oplus \cdots \oplus e_{s_{F_{1}}} \bigoplus_{1}^{E_{1}} e_{\infty} \bigoplus_{1}^{Z_{1}} 0$ as follows ( $\sim$ will denote unitary equivalence in the following calculation; all 
118

variables are defined as in Case 2 of Section 4.2):

$$
\begin{aligned}
& \operatorname{Std}\left(t_{1}^{\prime}, \ldots, t_{F_{2}}^{\prime}, E_{2}, Z_{2}\right) \\
& \sim e_{t_{2}} \oplus \cdots \oplus e_{t_{F_{2}}} \bigoplus_{1}^{E_{2}} e_{\infty} \bigoplus_{1}^{Z_{2}} 0 \\
& \sim \bigoplus_{1}^{l-1} e_{1} \oplus e_{t_{1}} \oplus \cdots \oplus e_{t_{F_{2}}} \bigoplus_{1}^{E_{2}} e_{\infty} \bigoplus_{1}^{Z_{1}} 0 \bigoplus_{1}^{Z} 0 \\
& \sim \quad \bigoplus_{1}^{a_{1}\left(k_{z_{2}}+1\right)+E_{1}-E_{2}} e_{1} \oplus e_{t_{1}^{\prime}} \oplus \cdots \oplus e_{t_{t_{2}^{\prime}}} \bigoplus_{1}^{E_{2}} e_{\infty} \bigoplus_{1}^{z_{1}} 0 \bigoplus_{1}^{\left(a_{1}+1\right)\left(k_{z_{2}}+1\right)+E_{1}-E_{2}} 0 \\
& \sim \bigoplus_{1}^{k_{Z_{2}}+1}\left(\bigoplus_{1}^{a_{1}} e_{1} \bigoplus_{1}^{a_{1}+1} 0\right) \bigoplus_{1}^{E_{1}-E_{2}}\left(e_{1} \oplus 0\right) \oplus e_{t_{1}} \oplus \cdots \oplus e_{t_{F_{2}}} \bigoplus_{1}^{E_{2}} e_{\infty} \bigoplus_{1}^{Z_{1}} 0 \\
& \bigoplus_{1}^{\left(k_{Z_{2}}+1\right)\left(a_{1}+1\right)} e_{0} \bigoplus_{1}^{E_{1}-E_{2}} e_{0} \oplus e_{t_{t}} \oplus \cdots \oplus e_{t_{F_{2}}} \bigoplus_{1}^{E_{2}} e_{\infty} \bigoplus_{1}^{E_{1}-E_{2}} e_{\infty} \bigoplus_{1}^{Z_{1}} 0 \\
& \sim \bigoplus_{1}^{Z} e_{0} \oplus e_{t_{t}} \oplus \cdots \oplus e_{t_{F_{2}}} \bigoplus_{1}^{E_{1}} e_{\infty} \bigoplus_{1}^{Z_{1}} 0 \\
& \sim e_{s_{1}^{\prime}} \oplus \cdots \oplus e_{s_{F_{1}}} \bigoplus_{1}^{E_{1}} e_{\infty} \bigoplus_{1}^{Z_{1}} 0 \text {. }
\end{aligned}
$$

Case 3: $Z_{1} \geq a_{1}+E_{1}$ and $Z_{2} \geq a_{1}+1-E_{2}$.

The argument is very similar to that given in Case 2. All variables are defined as in Case 4 of Section 4.2. In particular, define $t_{i}^{\prime}$ and $s_{i}^{\prime}$ as in Case 2 , but with $l=a_{1} k_{f}+E_{1}-E_{2}$. We need only check condition (iii) of Lemma 4.1.1, as conditions (i) and (ii) are clearly satisfied. Condition (iii) follows from the following calculation:

$$
\begin{aligned}
& \operatorname{Std}\left(t_{1}^{\prime}, \ldots, t_{F_{2}}^{\prime}, E_{2}, Z_{2}\right) \\
& \sim e_{t_{2}^{\prime}} \oplus \cdots \oplus e_{t_{F_{2}}} \bigoplus_{1}^{E_{2}} e_{\infty} \bigoplus_{1}^{Z_{2}} 0
\end{aligned}
$$




$$
\begin{aligned}
& \sim \bigoplus_{1}^{l-1} e_{1} \oplus e_{t_{l}^{\prime}} \oplus \cdots \oplus e_{t_{F_{2}}} \bigoplus_{1}^{E_{2}} e_{\infty} \bigoplus_{1}^{Z_{1}} 0 \bigoplus_{1}^{Z} 0 \\
& \sim \bigoplus_{1}^{a_{1} k_{f}+E_{1}-E_{2}} e_{1} \oplus e_{t_{2}^{\prime}} \oplus \cdots \oplus e_{t_{F_{2}}} \bigoplus_{1}^{E_{2}} e_{\infty} \bigoplus_{1}^{Z_{1}} 0 \bigoplus_{1}^{\left(a_{1}+1\right) k_{f}+E_{1}-E_{2}} 0 \\
& \sim \bigoplus_{1}^{k_{f}}\left(\bigoplus_{1}^{a_{1}} e_{1} \bigoplus_{1}^{a_{1}+1} 0\right) \bigoplus_{1}^{E_{1}-E_{2}}\left(e_{1} \oplus 0\right) \oplus e_{t_{l}} \oplus \cdots \oplus e_{t_{F_{2}}} \bigoplus_{1}^{E_{2}} e_{\infty} \bigoplus_{1}^{Z_{1}} 0 \\
& \sim \bigoplus_{1}^{k_{f}\left(a_{1}+1\right)} e_{0} \bigoplus_{1}^{E_{1}-E_{2}} e_{0} \oplus e_{t_{l}} \oplus \cdots \oplus e_{t_{F_{2}}} \bigoplus_{1}^{E_{2}} e_{\infty} \bigoplus_{1}^{E_{1}-E_{2}} e_{\infty} \bigoplus_{1}^{z_{1}} 0 \\
& \sim \bigoplus_{1}^{Z} e_{0} \oplus e_{t_{t}} \oplus \cdots \oplus e_{t_{F_{2}}} \bigoplus_{1}^{E_{1}} e_{\infty} \bigoplus_{1}^{Z_{1}} 0 \\
& \sim e_{s_{1}^{\prime}} \oplus \cdots \oplus e_{s_{F_{1}}^{\prime}} \bigoplus_{1}^{E_{1}} e_{\infty} \bigoplus_{1}^{Z_{1}} 0
\end{aligned}
$$

This completes the first step of the proof.

The second step of the proof is to show that $\operatorname{Std}\left(t_{1}^{\prime}, \ldots, t_{F_{2}}^{\prime}, E_{2}, Z_{2}\right)=$ $\operatorname{Std}\left(s_{1}^{\prime}, \ldots, s_{F_{1}}^{\prime}, E_{1}, Z_{1}\right)$. We will prove this using the continuity of standard maps and the map Std.

Define $t_{i, \varepsilon}^{\prime \prime}$ as follows:

$$
t_{i, \varepsilon}^{\prime \prime}=\left\{\begin{aligned}
\max \left(t_{d+1}^{\prime}, 1-\varepsilon\right) & \text { for } 1 \leq i \leq d \\
t_{i}^{\prime} & \text { otherwise }
\end{aligned}\right.
$$

Then the eigenvalues of $\operatorname{Std}\left(t_{1, \varepsilon}^{\prime \prime}, \ldots, t_{F_{2}, \varepsilon}^{\prime \prime}, E_{2}, Z_{2}\right)(h)$ and $\operatorname{Std}\left(t_{1}^{\prime}, \ldots, t_{F_{2}}^{\prime}, E_{2}, Z_{2}\right)(h)$ can be matched within $\varepsilon$. From step one we know that the eigenvalues of $\operatorname{Std}\left(t_{1}^{\prime}, \ldots, t_{F_{2}}^{\prime}, E_{2}, Z_{2}\right)(h)$ and $\operatorname{Std}\left(s_{1}^{\prime}, \ldots, s_{F_{1}}^{\prime}, E_{1}^{\prime}, Z_{1}\right)(h)$ are the same. Therefore the eigenvalues of $\operatorname{Std}\left(t_{1, \varepsilon}^{\prime \prime}, \ldots, t_{F_{2}, \varepsilon}^{\prime \prime}, E_{2}, Z_{2}\right)(h)$ can be matched within $\varepsilon$ with the eigenvalues of $\operatorname{Std}\left(s_{1}^{\prime}, \ldots, s_{F_{1}}^{\prime}, E_{1}, Z_{1}\right)(h)$. We can now apply Lemma 
4.2.1 to conclude that as $\varepsilon \rightarrow 0$,

$$
\operatorname{Std}\left(t_{1, \varepsilon}^{\prime \prime}, \ldots, t_{F_{2}, \varepsilon}^{\prime \prime}, E_{2}, Z_{2}\right)(f) \rightarrow \operatorname{Std}\left(s_{1}^{\prime}, \ldots, s_{F_{1}}^{\prime}, E_{1}, Z_{1}\right)(f)
$$

for all $f \in A_{1}$. From the construction of the map $\operatorname{Std}(\bullet, \ldots, \bullet, E, Z)$, it is clearly continuous in the parameters represented by a "๑" when $E$ and $Z$ are held fixed. Therefore as $\varepsilon \rightarrow 0$,

$$
\operatorname{Std}\left(t_{1, \varepsilon}^{\prime \prime}, \ldots, t_{F_{2}, \varepsilon}^{\prime \prime}, E_{2}, Z_{2}\right)(f) \rightarrow \operatorname{Std}\left(t_{1}^{\prime}, \ldots, t_{F_{2}}^{\prime}, E_{2}, Z_{2}\right)(f)
$$

for all $f \in A_{1}$. From the above argument it follows that

$$
\operatorname{Std}\left(t_{1}^{\prime}, \ldots, t_{F_{2}}^{\prime}, E_{2}, Z_{2}\right)(f)=\operatorname{Std}\left(s_{1}^{\prime}, \ldots, s_{F_{1}}^{\prime}, E_{1}, Z_{1}\right)(f)
$$

for all $f \in A_{1}$.

We can now proceed with the proof of Case 2.

Applying Proposition 4.3.3 to the maps $\left(f_{\infty} \phi\right)^{\prime}$ and $\left(f_{\infty} \psi\right)^{\prime}\left(\right.$ where $\left(f_{\infty} \phi\right)^{\prime}$ and $\left(f_{\infty} \psi\right)^{\prime}$ denote the maps $f_{\infty} \phi$ and $f_{\infty} \psi$ in standard form, respectively) yields a map $\gamma$ and an integer $k \geq 0$ such that

$$
\begin{aligned}
\gamma & =\operatorname{Std}\left(E t_{1}^{\prime}, \ldots, E t_{E F_{2}}^{\prime}, E E_{2}, E Z_{2}\right) \\
& =\operatorname{Std}\left(E s_{1}^{\prime}, \ldots, E s_{E F_{1}}^{\prime}, E E_{1}, E Z_{1}\right)
\end{aligned}
$$

and $E t_{i}^{\prime}=1,\left|E t_{i}^{\prime}-E t_{i}\right|<\delta$ for $i=1, \ldots, k, E t_{i}^{\prime}=E t_{i}$ for $i>k$,

$$
\left|E s_{i}^{\prime}-E s_{i}\right|<2 \delta
$$

for $i=1, \ldots, E F_{1}$ and $1 \geq E s_{1}^{\prime} \geq \cdots \geq E s_{E F_{1}}^{\prime} \geq 0, E E_{1}, E Z_{1}$ and $\gamma$ satisfy the conclusions of Lemma 4.1.1. 
By Case 1, there exists unitaries $V_{1}$ and $V_{2}$ such that $\operatorname{Ad}_{V_{1}} f_{1} \phi^{\prime}=\bigoplus_{1}^{a_{2}+1}\left(f_{\infty} \phi\right)^{\prime}$, $\operatorname{Ad}_{V_{2}}\left(\bigoplus_{1}^{a_{2}+1} \gamma\right)^{\prime}=\bigoplus_{1}^{a_{2}+1} \gamma$ and $\left\|V_{1}-V_{2}\right\|<4 \varepsilon$, where $\left(\bigoplus_{1}^{a_{2}+1} \gamma\right)^{\prime}$ denotes the map $\bigoplus_{1}^{a_{2}+1} \gamma$ in standard form.

Let $\kappa=\max \left(k, \min \left\{i: E t_{i}<1\right\}\right)$. By Lemma 4.3.3, $p \gamma p=p\left(f_{\infty} \psi\right)^{\prime} p$, where $p \in C_{2}$ is the support projection of the subrepresentation $e_{E t_{\kappa+1}} \oplus$ $\cdots \oplus e_{E t_{E F_{2}}}$. Again, by Lemma 4.3.3, $q\left(\oplus_{1}^{\alpha_{2}+1} \gamma\right)^{\prime} q=q f_{1} \psi^{\prime} q$, where $q=$ $\operatorname{Ad}_{V_{2}^{-}}\left(\bigoplus_{1}^{a_{2}+1} p\right)$ is the support projection of the subrepresentation $\bigoplus_{1}^{a_{2}+1}\left(e_{E t_{\kappa+1}} \oplus\right.$ $\left.\cdots \oplus e_{E T_{E F_{2}}}\right)$. Let $P=\left(\bigoplus_{1}^{a_{2}+1} p\right)$. Then

$$
\begin{aligned}
\operatorname{PAd}_{V_{2}}\left(f_{1} \psi^{\prime}\right) P & =\operatorname{Ad}_{V_{2}} q f_{1} \psi^{\prime} q \\
& =\operatorname{Ad}_{V_{2}} q\left(\bigoplus_{1}^{a_{2}+1} \gamma\right)^{\prime} q \\
& =P \operatorname{Pd}_{V_{2}}\left(\bigoplus_{1}^{a_{2}+1} \gamma\right)^{\prime} P \\
& =P \bigoplus_{1}^{a_{2}+1} \gamma P \\
& =\bigoplus_{1}^{a_{2}+1} p \gamma p \\
& =\bigoplus_{1}^{a_{2}+1} p\left(f_{\infty} \psi\right)^{\prime} p \\
& =P \bigoplus_{1}^{a_{2}+1}\left(f_{\infty} \psi\right)^{\prime} P .
\end{aligned}
$$

The above calculation demonstrates that the unitary $V_{2}$ conjugates the map $f_{1} \psi^{\prime}$ to $\bigoplus_{1}^{a_{2}+1}\left(f_{\infty} \psi\right)^{\prime}$ - at least restricted to the projection $P$. We will now consider the part supported on $P^{\perp}$.

By equations (4.43) and (4.70), $\left\|\gamma(f)-\left(f_{\infty} \psi\right)^{\prime}(f)\right\|<14 \varepsilon$ and $\|\left(\bigoplus_{1}^{\alpha_{2}+1} \gamma\right)^{\prime}(f)-$ 
$f_{\mathrm{I}} \psi^{\prime}(f) \|<14 \varepsilon$ for all $f \in F$. It follows that

$$
\begin{aligned}
& \left\|P^{\perp} \operatorname{Ad}_{V_{1}} f_{1} \psi^{\prime}(f) P^{\perp}-P^{\perp} \bigoplus_{1}^{a_{2}+1}\left(f_{\infty} \psi\right)^{\prime}(f) P^{\perp}\right\| \\
& \leq\left\|\operatorname{Ad}_{V_{1}} f_{1} \psi^{\prime}(f)-\bigoplus_{1}^{a_{2}+1}\left(f_{\infty} \psi\right)^{\prime}(f)\right\| \\
& \leq\left\|\operatorname{Ad}_{V_{1}} f_{1} \psi^{\prime}(f)-\operatorname{Ad}_{V_{1}} \bigoplus_{1}^{a_{2}+1} \gamma(f)\right\|+\left\|\bigoplus_{1}^{a_{2}+1} \gamma(f)-\bigoplus_{1}^{a_{2}+1}\left(f_{\infty} \psi\right)^{\prime}(f)\right\| \\
& \leq\left\|\operatorname{Ad}_{V_{1}} f_{1} \psi^{\prime}(f)-\operatorname{Ad}_{V_{2}^{*}}^{a_{2}+1} \bigoplus_{1}^{a_{2}+1} \gamma(f)\right\|+\left\|\operatorname{Ad}_{V_{2}^{*}} \bigoplus_{1}^{a_{1}} \gamma(f)-\operatorname{Ad}_{V_{1}^{*}} \bigoplus_{1}^{a_{2}+1} \gamma(f)\right\|+14 \varepsilon \\
& \leq 14 \varepsilon+8 \varepsilon+14 \varepsilon=36 \varepsilon
\end{aligned}
$$

for all $f \in F$. As $\left\|V_{1}-V_{2}\right\|<4 \varepsilon$, by the above calculation

$$
\left\|P^{\perp} \operatorname{Ad}_{V_{2}} f_{1} \psi^{\prime}(f) P^{\perp}-P^{\perp} \bigoplus_{1}^{a_{2}+1}\left(f_{\infty} \psi\right)^{\prime}(f) P^{\perp}\right\|<44 \varepsilon
$$

for all $f \in F$. As $\left|E t_{i}-1\right|<\delta$ for $i=1, \ldots, k$, for every irreducible subrepresentation $e_{t}$ of $P^{\perp} \operatorname{Ad}_{V_{2}} f_{1} \psi^{\prime} P^{\perp}$ and $P^{\perp} \bigoplus_{1}^{a_{2}+1}\left(f_{\infty} \psi\right)^{\prime} P^{\perp}$,

$$
t=\infty,|t-0|<\delta \text {, or }|t-1|<\delta \text {. }
$$

We may now apply Lemma 4.3.2 to find a unitary path $V_{t}^{\prime}$ supported on $P^{\perp}$ such that

$$
\operatorname{Ad}_{V_{1}^{\prime}} P^{\perp} \operatorname{Ad}_{V_{2}} f_{1} \psi^{\prime} P^{\perp}=P^{\perp} \bigoplus_{1}^{a_{2}+1}\left(f_{\infty} \psi\right)^{\prime} P^{\perp}
$$

and

$$
\left\|\operatorname{Ad}_{V_{t}^{\prime}} P^{\perp} \operatorname{Ad}_{V_{2}} f_{1} \psi^{\prime}(f) P^{\perp}-P^{\perp} \bigoplus_{1}^{a_{2}+1}\left(f_{\infty} \psi\right)^{\prime}(f) P^{\perp}\right\|<19 \cdot 44 \varepsilon<1000 \varepsilon
$$

for $t \in[0,1]$ and $f \in F$. Then $\operatorname{Ad}_{V_{1}^{\prime} V_{2}} f_{1} \psi^{\prime}=\bigoplus_{1}^{a_{2}+1}\left(f_{\infty} \psi\right)^{\prime}, \| \operatorname{Ad}_{V_{t}^{\prime} V_{2}} f_{1} \psi^{\prime}(f)-$ $\bigoplus_{1}^{a_{2}+1}\left(f_{\infty} \psi\right)^{\prime}(f) \|<1000 \varepsilon, \operatorname{Ad}_{V_{1}} f_{1} \phi^{\prime}=\bigoplus_{1}^{\alpha_{2}+1}\left(f_{\infty} \phi\right)^{\prime}$ and $\left\|V_{1}-V_{2}\right\|<4 \varepsilon$. 
For the right endpoint, the above arguments yield unitaries $V_{1 R} \equiv V_{1}, V_{2 R} \equiv$ $V_{2}$ and a unitary path $V_{R, t}^{\prime} \equiv V_{t}^{\prime}$ such that

$$
\begin{gathered}
\operatorname{Ad}_{V_{1 R}} f_{1} \phi^{\prime}=\bigoplus_{1}^{a_{2}+1}\left(f_{\infty} \phi\right)^{\prime} \\
\operatorname{Ad}_{V_{R, 1}^{\prime}} V_{2 R} f_{1} \psi^{\prime}=\bigoplus_{1}^{a_{2}+1}\left(f_{\infty} \psi\right)^{\prime}, \\
\left\|V_{1 R}-V_{2 R}\right\|<4 \varepsilon
\end{gathered}
$$

and

$$
\left\|\operatorname{Ad}_{V_{R, \varepsilon}^{\prime} V_{2 R}} f_{1} \psi^{\prime}(f)-\bigoplus_{1}^{a_{2}+1}\left(f_{\infty} \psi\right)^{\prime}(f)\right\|<1000 \varepsilon
$$

Similarly, at the left endpoint we can find unitaries $V_{1 L}, V_{2 L}$ and a unitary path $V_{L, t}^{\prime}$ satisfying a similar set of equations. Let $U_{1, t}$ and $U_{2, t}^{\prime}$ be any path joining $V_{1 L}$ to $V_{1 R}$ and $V_{2 L}$ to $V_{2 R}$ respectively such that $\left\|U_{1, t}-U_{2, t}^{\prime}\right\|<4 \varepsilon$ for all $t \in[0,1]$ and locally constant at the endpoints;

$$
U_{1, t}=\left\{\begin{array}{lll}
U_{1,0} & \text { for } & t \in[0, \delta] \\
U_{1,1} & \text { for } & t \in[1-\delta, 1]
\end{array}\right.
$$

and

Finally, define $U_{2, t}$ as follows:

$$
U_{2, t}^{\prime}=\left\{\begin{array}{lll}
U_{2,0}^{\prime} & \text { for } & t \in[0, \delta] \\
U_{2,1}^{\prime} & \text { for } & t \in[1-\delta, 1]
\end{array}\right.
$$

$$
U_{2, t}=\left\{\begin{array}{rll}
V_{L, 1-t / \delta}^{\prime} U_{2, t}^{\prime} & \text { for } & t \in[0, \delta] \\
U_{2, t}^{\prime} & \text { for } & t \in[\delta, 1-\delta] \\
V_{R,(t+\delta-1) / \delta} U_{2, t}^{\prime} & \text { for } & t \in[1-\delta, 1] .
\end{array}\right.
$$

Then for $t \in[\delta, 1-\delta]$ and $f \in F$, by equation (4.43) and the above definition for $U_{1, t}$ and $U_{2, t}$,

$$
\left\|\operatorname{Ad}_{U_{1, t}} \phi^{\prime}(f)(t)-\operatorname{Ad}_{U_{2, t}} \psi^{\prime}(f)(t)\right\|
$$


124

$$
\begin{aligned}
& \leq\left\|\operatorname{Ad}_{U_{2, t}^{*} U_{1, t}} \phi^{\prime}(f)(t)-\phi^{\prime}(f)(t)\right\|+\left\|\phi^{\prime}(f)(t)-\psi^{\prime}(f)(t)\right\| \\
& \leq 8 \varepsilon+7 \varepsilon=15 \varepsilon .
\end{aligned}
$$

For $t \in[1-\delta, 1]$ and $f \in F$,

$$
\begin{aligned}
&\left\|\operatorname{Ad}_{U_{1, t}} \phi^{\prime}(f)(t)-\operatorname{Ad}_{U_{2, t}} \psi^{\prime}(f)(t)\right\| \\
&=\left\|\operatorname{Ad}_{V_{1}} \phi^{\prime}(f)(t)-\operatorname{Ad}_{V_{R,(t+\delta-1) / \delta} V_{2 R}} \psi^{\prime}(f)(t)\right\| \\
& \leq\left\|\operatorname{Ad}_{V_{1}} f_{1} \phi^{\prime}(f)-\operatorname{Ad}_{V_{R_{,}(t+\delta-1) / \delta} V_{2 R}} f_{1} \psi^{\prime}(f)\right\|+2 \varepsilon \\
& \leq\left\|\bigoplus_{1}^{a_{2}+1}\left(f_{\infty} \phi\right)^{\prime}(f)-\bigoplus_{1}^{a_{2}+1}\left(f_{\infty} \psi\right)^{\prime}(f)\right\| \\
&+\left\|\bigoplus_{1}^{a_{2}+1}\left(f_{\infty} \psi\right)^{\prime}(f)-\operatorname{Ad}_{V_{R_{,},(t+\delta-1) / \delta} V_{2 R}} f_{1} \psi^{\prime}(f)\right\|+2 \varepsilon \\
& \leq 7 \varepsilon+1000 \varepsilon+2 \varepsilon=1009 \varepsilon .
\end{aligned}
$$

A similar calculation holds for the $t \in[0, \delta]$.

\subsection{Jiang and Su's argument}

We will use Lemma 5.1 of [JS] and the application of the lemma as described in Theorem 5.2 of [JS], to construct unitaries $V_{1}$ and $V_{2}$ in the unitization of $A_{2}$ such that

$$
\left\|\operatorname{Ad}_{V_{1}} \phi(f)-\operatorname{Ad}_{U_{1}} \phi^{\prime}(f)\right\|<\varepsilon
$$

and

$$
\left\|\operatorname{Ad}_{V_{2}} \psi(f)-\operatorname{Ad}_{U_{2}} \psi^{\prime}(f)\right\|<\varepsilon
$$

for $f \in F$. The conclusion of the uniqueness theorem will then follow from an elementary argument involving the unitaries $V_{1}$ and $V_{2}$, and the results of 
section 4.3.

The following lemma is Lemma 5.1 of [JS]:

Lemma 4.4.1 Let $m$ be a positive integer, let $D \subset M_{m}(\mathbb{C})$ be a unital sub$C^{*}$-algebra, and let $F \subset D$ be a finite subset which contains a set of generators of $D$. For any $1 / 9>\varepsilon>0$, there exists $\delta>0$ such that if $R, S \in M_{m}(\mathbb{C})$ are two unitaries satisfying

$$
\left\|R f R^{*}-S f S^{*}\right\|<\delta, f \in F,
$$

then there exists a unitary path $\{X(t) \mid t \in[0,1]\}$ in $M_{m}(\mathbb{C})$ with $X(0)=R$ and $X(1)=S$ satisfying

$$
\left\|X(t) f X(t)^{*}-S f S^{*}\right\|<\varepsilon, f \in F
$$

In our application of the lemma the subalgebra $D$ (referred to in the statement above) may be non-unital; in this case we will consider instead its unitization.

We will construct the unitary $V_{1}(t), t \in[0,1]$ piecewise as follows. Given $\delta>0$ as in the conclusion of the above lemma, we can find a $\delta^{\prime}>0$ such that

$$
\left\|\operatorname{Ad}_{U_{1}} \phi^{\prime}(f)(x)-\operatorname{Ad}_{U_{1}} \phi^{\prime}(f)(y)\right\|<\delta / 2
$$

and

$$
\|\phi(f)(x)-\phi(f)(y)\|<\delta / 2
$$

for all $f \in F$ if $|x-y|<\delta^{\prime}$. Let $\left\{t_{i}\right\}_{i=1}^{N}$ be a partition of $[0,1]$ such that $\left|t_{i}-t_{i+1}\right|<\delta^{\prime}$ and $t_{1}=0, t_{N}=1$. Let $V_{1}\left(t_{i}\right)$ for $i \neq 1, N$ be any unitary 
in $D_{2}$ such that $\operatorname{Ad}_{V_{1}\left(t_{i}\right)} \phi(f)\left(t_{i}\right)=\operatorname{Ad}_{U_{1}} \phi^{\prime}(f)\left(t_{i}\right)$. For $i=1$ we can choose $V_{1}\left(t_{0}\right)$ to be contained in the image of the inclusion $\bigoplus_{1}^{a_{2}} C_{2} \hookrightarrow D_{2}$ as both $\phi(f)\left(t_{1}\right)$ and $\operatorname{Ad}_{U_{1}} \phi^{\prime}(f)\left(t_{1}\right)$ are contained in this subalgebra. Similarly for $i=N$ we can choose $V_{1}\left(t_{N}\right)$ to be contained in the image of the inclusion $\bigoplus_{1}^{a_{2}+1} C_{2} \hookrightarrow D_{2}$. By equations 4.73 and 4.74 ,

$$
\left\|\operatorname{Ad}_{V_{1}\left(t_{i}\right)} \phi(f)\left(t_{i}\right)-\operatorname{Ad}_{V_{1}\left(t_{i+1}\right)} \phi(f)\left(t_{i}\right)\right\|<\delta
$$

for all $f \in F$. Therefore, by Lemma 5.1 of [JS], there exists a unitary path joining $V_{1}\left(t_{i}\right)$ to $V_{1}\left(t_{i+1}\right)$ such that $\left\|\operatorname{Ad}_{V_{1}(t)} \phi(f)\left(t_{i}\right)-\operatorname{Ad}_{V_{1}\left(t_{i+1}\right)} \phi(f)\left(t_{i}\right)\right\|<\varepsilon$ for all $f \in F, t \in\left[t_{i}, t_{i+1}\right]$. We have constructed a unitary path on each subinterval $\left[t_{i}, t_{i+1}\right]$. Furthermore, it can be easily checked that $\| \operatorname{Ad}_{V_{1}} \phi(f)-$ $\operatorname{Ad}_{U_{1}} \phi^{\prime}(f) \|<\varepsilon$ for all $f \in F$. Similarly, we can construct a unitary $V_{2}$ such that $\left\|\operatorname{Ad}_{V_{2}} \psi(f)-\operatorname{Ad}_{U_{2}} \psi^{\prime}(f)\right\|<\varepsilon$.

Finally, we can construct the unitary $U$ that is the essential content of the uniqueness theorem. Let $U=V_{1}^{*} V_{2}$, then for $f \in F$,

$$
\begin{aligned}
&\left\|\phi(f)-U \psi(f) U^{*}\right\| \\
&=\left\|\operatorname{Ad}_{V_{1}} \phi(f)-\operatorname{Ad}_{V_{2}} \psi(f)\right\| \\
& \leq\left\|\operatorname{Ad}_{V_{1}} \phi(f)-\operatorname{Ad}_{U_{1}} \phi^{\prime}(f)\right\|+\left\|\operatorname{Ad}_{U_{1}} \phi^{\prime}(f)-\operatorname{Ad}_{V_{2}} \psi^{\prime}(f)\right\| \\
&+\left\|\operatorname{Ad}_{U_{2}} \psi^{\prime}(f)-\operatorname{Ad}_{V_{2}} \psi(f)\right\| \\
& \leq \varepsilon+1009 \varepsilon+\varepsilon=1011 \varepsilon .
\end{aligned}
$$




\section{Bibliography}

[Bla] B. Blackadar, A simple $\mathrm{C}^{*}$-algebra with no nontrivial projections, Proc. Amer. Math. Soc. 78 (1980), 504-508.

[Dea] A. Dean, A continuous field of projectionless $\mathrm{C}^{*}$-algebras, preprint.

[El11] G. A. Elliott, On the classification of inductive limits of sequences of semisimple finite-dimensional algebras, J. Algebra 38 (1976), 29-44.

[Ell2] G. A. Elliott, On the classification of $C^{*}$-algebras of real rank zero, $J$. Reine Angew. Math. 443 (1993), 179-219.

[El13] G. A. Elliott, A classification of certain simple $C^{*}$-algebras, in Quantum and Non-Commutative Analysis (H. Araki et al. eds.), Kluwer, Dordrecht, 1993, pp. 373-385.

[Ell4] G. A. Elliott, A classification of certain simple $C^{*}$-algebras II, $J$. Ramanujan Math. Soc. 12 (1997), 97-134.

[El15] G. A. Elliott, The classification problem for amenable $C^{*}$-algebras, Proceedings of the International Congress of Mathematicians, Zürich, Switzerland, 1994 (editor, S.J. Chatterji), Birkhäuser Verlag, Basel, 1995, pp. 922-932. 
[Ell6] G. A. Elliott, An invariant for simple $C^{*}$-algebras, Invited $P a$ pers/Articles sollicités, Canadian Mathematical Society 1945-1995, Volume 3 (edited by J. B. Carrell and R. Murty), Canadian Mathematical Society, Ottawa, 1996, pages 61-90.

[EGL] G. A. Elliott, G. Gong, L. Li, On simple inductive limits of matrix algebras over higher dimensional spaces, II, preprint.

[EGJS] G. A. Elliott, G. Gong, X. Jiang, and H. Su, A classification of simple limits of dimension drop $C^{*}$-algebras, Fields Institute Communications 13 (1997), 125-143.

[EV] G. A. Elliott and J. Villadsen, Perforated $K_{0}$-groups, Canad. J. Math., to appear.

[JS] X. Jiang and $\mathrm{H}$. Su, On a simple unital projectionless $C^{*}$-algebra, preprint.

[Kir] E. Kirchberg, The classification of purely infinite $\mathrm{C}^{*}$-algebras using Kasparov's theory, preprint.

[KK] A. Kishimoto, A. Kumjian, Simple stably projectionless $\mathrm{C}^{*}$-algebras arising as crossed products, Canad. J. Math. 48 (1996) \# 5, 980-996.

[Li] L. Li, Simple inductive limit $C^{*}$-algebras: Spectra and approximation by interval algebras, J. Reine Angew. Math. 507 (1999), 57-79.

[NT] K. E. Nielsen, and K. Thomsen, Limits of circle algebras, Expo. Math. 14 (1996), 17-56. 
- [Phi] N. C. Phillips, A classification theorem for nuclear purely infinite $\mathrm{C}^{*}$ algebras, Doc. Math. 5 (2000), 49-114.

[Ste] I. Stevens, Hereditary subalgebras of certain simple non real rank zero $C^{*}$-algebras, Fields Institute Monographs 13 (1999), 207-242.

[T] K. Thomsen, Inductive limits of interval algebras: The tracial state space, Amer. J. Math 116 (1994), no. 3, 605-620. 